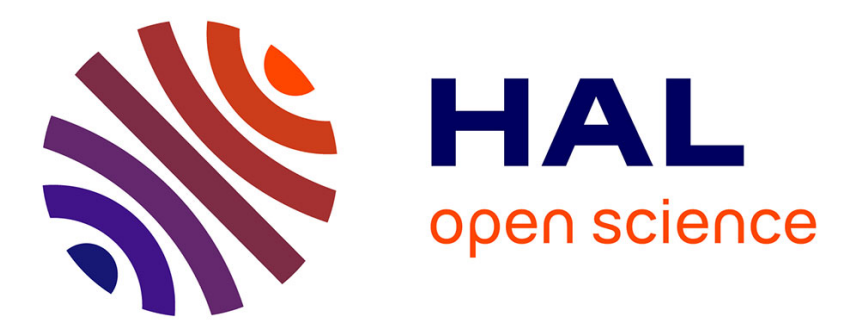

\title{
Corner asymptotics of the magnetic potential in the eddy-current model
}

Monique Dauge, Patrick Dular, Laurent Krähenbühl, Victor Péron, Ronan

Perrussel, Clair Poignard

\section{To cite this version:}

Monique Dauge, Patrick Dular, Laurent Krähenbühl, Victor Péron, Ronan Perrussel, et al.. Corner asymptotics of the magnetic potential in the eddy-current model. Mathematical Methods in the Applied Sciences, 2014, 37 (13), pp.1924-1955. 10.1002/mma.2947 . hal-00779067

\section{HAL Id: hal-00779067 \\ https://hal.inria.fr/hal-00779067}

Submitted on 21 Jan 2013

HAL is a multi-disciplinary open access archive for the deposit and dissemination of scientific research documents, whether they are published or not. The documents may come from teaching and research institutions in France or abroad, or from public or private research centers.
L'archive ouverte pluridisciplinaire HAL, est destinée au dépôt et à la diffusion de documents scientifiques de niveau recherche, publiés ou non, émanant des établissements d'enseignement et de recherche français ou étrangers, des laboratoires publics ou privés. 


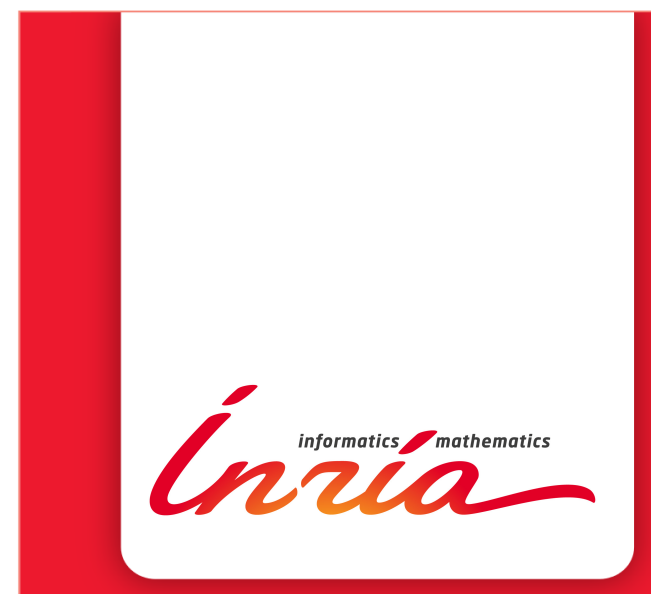

\section{Corner asymptotics of the magnetic \\ potential in the eddy-current model}

M. Dauge ${ }^{1}$, P. Dular ${ }^{2}$, L. Krähenbühl ${ }^{3}$, V. Péron ${ }^{4}$, R. Perrussel $^{5}$ and C. Poignard $^{6}$

${ }^{1}$ IRMAR CNRS UMR6625, Rennes, France

${ }^{2}$ F.R.S.-FNRS, ACE research unit, Liège, Belgium

${ }^{3}$ Laboratoire Ampère CNRS UMR5005, Lyon, France

${ }^{4}$ LMAP CNRS UMr5142 \& Team M3D INRIA, Université de Pau, Pau, France

${ }^{5}$ LAPLACE CNRS UMR5213, Toulouse, France

${ }^{6}$ Team MC2, INRIA Bordeaux-Sud-Ouest \& CNRS UMR 5251, Bordeaux, France

\section{RESEARCH}

\section{REPORT}

$\mathrm{N}^{\circ} 8204$

January 2013

Project-Teams Magique-3D and MC2 



\title{
Iniva
}

\section{Corner asymptotics of the magnetic potential in the eddy-current model}

\author{
M. Dauge ${ }^{1}$, P. Dular ${ }^{2}$, L. Krähenbühl ${ }^{3}$, V. Péron ${ }^{4}$, R. Perrussel ${ }^{5}$ \\ and C. Poignard ${ }^{6}$ \\ ${ }^{1}$ IRMAR CNRS UMR6625, Rennes, France \\ ${ }^{2}$ F.R.S.-FNRS, ACE research unit, Liège, Belgium \\ ${ }^{3}$ Laboratoire Ampère CNRS UMR5005, Lyon, France \\ ${ }^{4}$ LMAP CNRS UMR5142 \& Team M3D INRIA, Université de Pau, Pau, France \\ ${ }^{5}$ LAPLACE CNRS UMR5213, Toulouse, France \\ 6 Team MC2, INRIA Bordeaux-Sud-Ouest \& CNRS UMR 5251, Bordeaux, France
}

Project-Teams Magique-3D and MC2 
Abstract: In this paper, we describe the scalar magnetic potential in the vicinity of a corner of a conducting body embedded in a dielectric medium in a bidimensional setting. We make explicit the corner asymptotic expansion for this potential as the distance to the corner goes to zero. This expansion involves singular functions and singular coefficients. We introduce a method for the calculation of the singular functions near the corner and we provide two methods to compute the singular coefficients: the method of moments and the method of quasi-dual singular functions. Estimates for the convergence of both approximate methods are proven. We eventually illustrate the theoretical results with finite element computations. The specific non-standard feature of this problem lies in the structure of its singular functions: They have the form of series whose first terms are harmonic polynomials and further terms are genuine non-smooth functions generated by the piecewise constant zeroth order term of the operator.

Key-words: Corner asymptotics, Calculus of singular functions, Magnetic potential, Eddy current problem 


\section{Asympotiques de coin pour le potentiel magnétique dans le problème des courants de Foucault}

Résumé : Dans cet article, nous décrivons le potentiel scalaire magnétique solution du problème bidimensionnel des courants de Foucault au voisinage du coin d'un domaine conducteur immergé dans un milieu diélectrique. Nous explicitons l'asymptotique de coins de ce potentiel lorsque la distance au coin tend vers zéro. Cette asymptotique nécessite le calcul des fonctions singulières et des coefficients de singularités. Nous proposons une méthode pour calculer récursivement les fonctions singulières. La méthode quasi-duale est présentée pour le calcul approché des coefficients de singularités et des estimations d'erreur sont prouvées. Nous illustrons ces résultats théoriques par des simulations par éléments finis. La caractéristique principale de ce travail réside dans la structure des fonctions singulières: l'inhomogénéité de l'opérateur d'ordre 2 et le fait que son terme d'ordre 0 soit constant par morceaux entraînent que les fonctions singulières sont obtenus sous forme de séries dont chaque premier terme est un polynôme harmonique et dont les termes suivant sont des fonctions analytiques par morceaux générés par le terme d'ordre 0 .

Mots-clés : Asymptotiques de coins, Calcul de fonctions singulières, Potentiel magnétique, Problème des courants de Foucault 


\section{Contents}

1 Introduction $\quad \mathbf{5}$

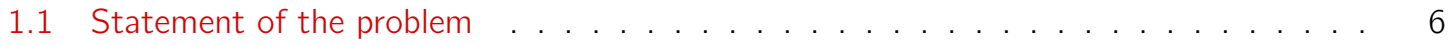

1.2 Corner asymptotics . . . . . . . . . . . . . . . . . . . . . . . . . . . 7

1.3 Outline of the paper . . . . . . . . . . . . . . . . . . . . . 8

2 Laplace operator $\quad \mathbf{8}$

2.1 The method of moments . . . . . . . . . . . . . . . . . . . . . . . . . . . . 9

2.2 The method of dual functions . . . . . . . . . . . . . . . . . . . . . . . . 10

3 The problem with angular conductor $\quad 10$

3.1 Principles of the construction of the primal singularities . . . . . . . . . . . . . . . . 12

3.2 Principles of the construction of the dual singularities . . . . . . . . . . . . . . . . 13

3.3 A first try to extract the singular coefficients: the method of moments . . . . . . . . 14

3.4 Extraction of singular coefficients by the method of quasi-dual functions . . . . . . . . 16

4 Leading singularities and their first shadows in complex variables 20

4.1 Formalism in complex variables . . . . . . . . . . . . . . . . . . . . . . . . . . . 20

4.2 Calculation of the shadow function generated by $z^{k}$ and $\log z \ldots \ldots 22$

4.3 Explicit expressions of the first shadows of primal singularities. . . . . . . . . . . . . . . . . 25

4.4 Explicit expressions of the first shadows of dual singularities . . . . . . . . . . . . 26

5 Numerical simulations $\quad 27$

6 Conclusion $\quad 32$

$\begin{array}{lr}\text { References } & 34\end{array}$

A Appendix: Tools for the derivation of the shadows at any order 35

A.1 Definition of the space $\mathbb{J}_{n}^{\lambda}$ on $\mathcal{G}$ and the congruence relation $\equiv$ on $\mathbb{S}_{\ell, n}^{\lambda}$ and $\mathbb{J}_{n}^{\lambda} \ldots . .36$

A.2 Generic elementary calculation . . . . . . . . . . . . . . . . . . 36

A.2.1 The case $\lambda \neq 0 \ldots \ldots \ldots \ldots \ldots \ldots \ldots$

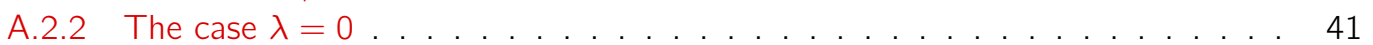

A.3 Formal derivation of the singularities . . . . . . . . . . . . . . . . . . . . . . . . . . . . . . 44

A.3.1 Principles . . . . . . . . . . . . . . . . . . . . . 44

A.3.2 Generic expression of the shadows at any order . . . . . . . . . . . . . . . . . 44 


\section{Introduction}

Accurate knowledge of eddy currents is of great interest for the design of many electromagnetic devices such as used in electrothermics. Taking advantage of the fast decrease of the electromagnetic field inside the conductor, impedance conditions are usually considered to reduce the computational domain. Impedance conditions were first proposed by Leontovich [13] and by Rytov [17] in the 1940's and then extended by Senior and Volakis [18]. These conditions can be derived up to the required precision for any conductor with a smooth surface: we refer to Haddar et al. [9] for the mathematical justification of conditions of order 1,2 and 3. Note however that such conditions are not valid near corners. Few authors have proposed heuristic impedance modifications close to the corners [6,21], but these modifications are neither satisfactory nor proved. In particular in [21], the modified impedance appears to blow up near the corner, which does not seem valid for non-magnetic materials with a finite conductivity as presented in [1].

In the present paper, we do not consider the derivation of the impedance condition, which is an asymptotic expansion in high frequency (or high conductivity). We are rather interested in the description of the magnetic potential in the vicinity of the corner of a conducting body embedded in a dielectric medium in a bidimensional setting, considering the conductivity $\sigma$, as well as the angular frequency $\kappa$, as given parameters. We emphasize that these considerations will be helpful in understanding the behavior of the impedance condition near corner singularities that will be considered in a forthcoming paper.

Roughly speaking, the aim of the present paper is to provide the asymptotic expansion of the magnetic potential as the distance to the corner goes to zero. This notion of corner asymptotics generalizes the Taylor expansion, which holds in smooth domains. Such asymptotics involve two main ingredients:

- The singular functions, also called singularities, which belong to the kernel of the considered operator.

- The singular coefficients, whose calculation requires the knowledge of (quasi-) dual singular functions.

In the present paper, we consider the magnetic potential in a non-magnetic domain composed of a conducting material $\Omega_{-}$with one corner surrounded by a dielectric material $\Omega_{+}$. Thus the operator acting on the magnetic potential is not homogeneous and has a discontinuous piecewise constant coefficient in front of its zeroth order part:

$$
-\Delta+\mathrm{i} \kappa \mu_{0} \sigma \mathbb{1}_{\Omega_{-}} .
$$

Though pertaining to the wide class of elliptic boundary value problems or transmission problems in conical domains, see for instance the papers $[10,11]$ and the monographs $[8,5,16,12]$, this problem has specific features which make the derivation of the asymptotics not obvious-namely the fact that singularities are generated by a non-principal term. Despite its great interest for the applications, this problem has not yet been explicitly analyzed.

Another disturbing factor is the nature of the limit problem when the product $\kappa \mu_{0} \sigma$ tends to infinity (large frequency/high conductivity limit). This limit is simply the homogeneous Dirichlet problem for the Laplace operator set in the dielectric medium $\Omega_{+}$, whose corner singularities are well known $[10,8]$. In particular, when the conductor $\Omega_{-}$has a convex corner, its surrounding domain $\Omega_{+}$has a non-convex corner, thus the Dirichlet problem has non $\mathcal{C}^{1}$ singularities, in opposition to the problem for any finite $\kappa \mu_{0} \sigma$. This apparent paradox can be solved by a delicate multi-scale analysis, whose heuristics are 
exposed in [1]. Roughly speaking, there exists profiles in $\mathbb{R}^{2}$ which have the singular behavior of the operator $-\Delta+2 i \mathbb{1}_{\Omega_{-}}$near the corner, and which connect at infinity with the singular functions of the Dirichlet problem in $\Omega_{+}$, as described by equation (13) in [1]. This is why the knowledge of the singularities of $-\Delta+\mathrm{i} \kappa \mu_{0} \sigma \mathbb{1}_{\Omega_{-}}$at given $\kappa$ and $\sigma$ is a milestone in the full multi-scale analysis.

Besides the mere description of singular functions, the computation of singular coefficients is considered in this paper. Various works concern the extraction of singular coefficients associated with the solution to an elliptic problem set in a domain with a corner singularity, see $[14,4]$ for theoretical formulas, and $[15,20,3]$ for more practical methods. Here, we choose to extend the quasi-dual function method initiated in [3] to the case of resonances, which was discarded in the latter reference.

Let us now present the problem considered throughout the paper.

\subsection{Statement of the problem}

Denote by $\Omega_{-} \subset \mathbb{R}^{2}$ the bounded domain corresponding to the conducting medium, and by $\Omega_{+} \subset \mathbb{R}^{2}$ the surrounding dielectric medium (see Figure 1(a)). The domain $\Omega$ with a smooth boundary $\Gamma$ is defined by $\Omega=\Omega_{-} \cup \Omega_{+} \cup \Sigma$, where $\Sigma$ is the boundary of $\Omega_{-}$. For the sake of simplicity, we assume that:

1. $\Sigma$ has only one geometric singularity, and we denote by $\mathbf{c}$ this corner. The angle of the corner (from the conducting material, see Figure $1(a)$ ) is denoted by $\omega$.

2. The current source term $J$, located in $\Omega_{+}$, is a smooth function, which vanishes in a neighborhood of $\mathbf{c}$.

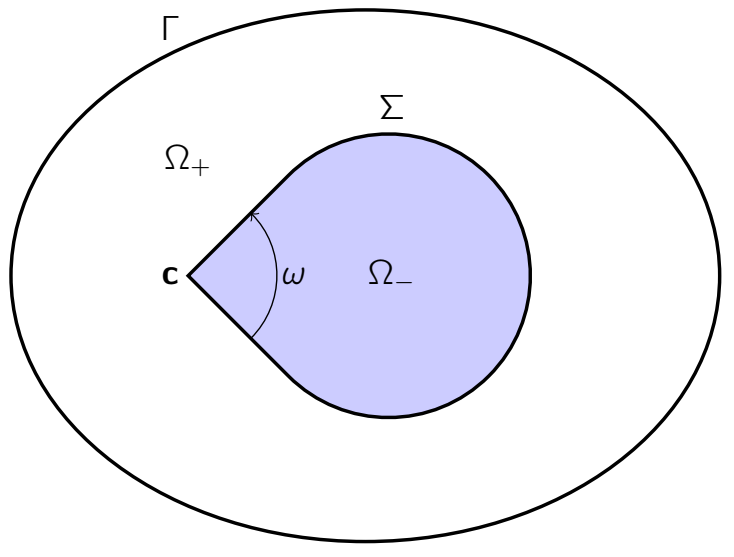

(a) Domain for the eddy-current problem.

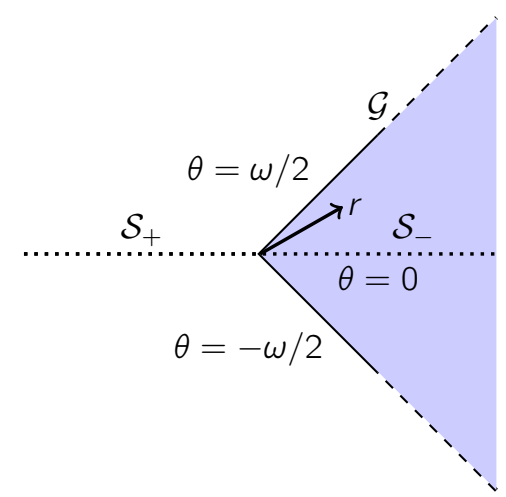

(b) Infinite sectors $\mathcal{S}_{+}$and $\mathcal{S}_{-}$in $\mathbb{R}^{2}$.

Figure 1: Geometries of the considered problems.

The magnetic vector potential $\mathcal{A}$ (reduced to a single scalar component in $2 \mathrm{~d}$ ) satisfies the following problem:

$$
\left\{\begin{aligned}
-\Delta \mathcal{A}^{+} & =\mu_{0} J \text { in } \Omega_{+}, & & {[\mathcal{A}]_{\Sigma}=0, } \\
-\Delta \mathcal{A}^{-}+\mathrm{i} \kappa \mu_{0} \sigma \mathcal{A}^{-} & =0 \text { in } \Omega_{-}, & & \text {on } \Sigma, \\
\mathcal{A}^{+} & =0 \text { on } \Gamma, & {\left[\partial_{n} \mathcal{A}\right]_{\Sigma}=0, } & \text { on } \Sigma,
\end{aligned}\right.
$$

where $\mathrm{i}$ is the imaginary unit, $\mu_{0}$ the vacuum permeability, $\kappa$ the angular frequency of the phenomenon and $\sigma$ the electric conductivity. Here, $\mathcal{A}^{ \pm}$denotes the restriction of $\mathcal{A}$ to $\Omega_{ \pm}$and $[\mathcal{A}]_{\Sigma}:=\left.\mathcal{A}^{+}\right|_{\Sigma}-\left.\mathcal{A}^{-}\right|_{\Sigma}$ 
is the jump of $\mathcal{A}$ across the interface $\Sigma$. Similarly, $\left[\partial_{n} \mathcal{A}\right]_{\Sigma}$ is the jump of the normal derivative of $\mathcal{A}$, where $n$ is the unit normal vector to $\Sigma$. Since we consider the parameters as given in the present purpose, for the sake of normalization we introduce the positive parameter $\zeta$ as

$$
\zeta^{2}=\kappa \mu_{0} \sigma / 4
$$

so that the problem satisfied by $\mathcal{A}$ writes

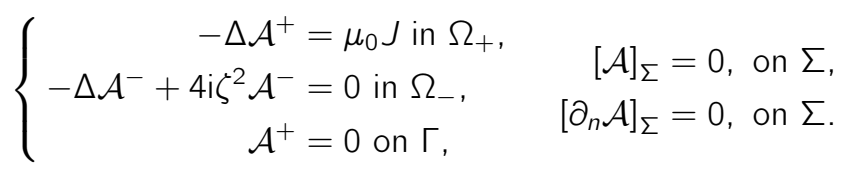

The factor 4 is present in order to simplify the forthcoming calculations (see equation (47)) in Section 4 and in Appendix-A.

The variational formulation of problem (1) is

$$
\left\{\begin{array}{l}
\text { Find } \mathcal{A} \in H_{0}^{1}(\Omega) \text { such that } \forall \mathcal{A}_{*} \in H_{0}^{1}(\Omega), \\
\int_{\Omega_{+}} \nabla \mathcal{A}^{+} \cdot \nabla \overline{\mathcal{A}_{*}^{+}} \mathrm{d} x \mathrm{~d} y+\int_{\Omega_{-}} \nabla \mathcal{A}^{-} \cdot \nabla \overline{\mathcal{A}_{*}^{-}}+4 i \zeta^{2} \mathcal{A}^{-} \overline{\mathcal{A}_{*}^{-}} \mathrm{d} x \mathrm{~d} y=\mu_{0} \int_{\Omega} J \overline{\mathcal{A}_{*}} \mathrm{~d} x \mathrm{~d} y .
\end{array}\right.
$$

Since this form is coercive on $H_{0}^{1}(\Omega)$, there exists a unique solution $\mathcal{A}$ in $H_{0}^{1}(\Omega)$ to problem (1). In addition, note that $\mathcal{A}$ is solution to the Dirichlet problem

$$
\left\{\begin{aligned}
-\triangle \mathcal{A} & =F \text { in } \Omega \\
\mathcal{A} & =0 \text { on } \Gamma
\end{aligned}\right.
$$

where $F=\mu_{0} J \mathbb{1}_{\Omega_{+}}-4 i \zeta^{2} \mathcal{A} \mathbb{1}_{\Omega_{-}}$, the functions $\mathbb{1}_{\Omega_{ \pm}}$being the characteristic functions of $\Omega_{ \pm}$. Since $J$ is smooth and since $\mathcal{A}$ belongs to $H^{1}(\Omega)$, then $F$ belongs to $L^{2}(\Omega)$, and even to $H^{\frac{1}{2}-\varepsilon}(\Omega)$ for any $\varepsilon>0$, - here the bound on the Sobolev exponent comes from the discontinuity of $F$ across $\Sigma$. Standard elliptic regularity of the Laplace operator implies that $\mathcal{A}$ belongs to $H^{2}(\Omega)$, and even to $H^{\frac{5}{2}-\varepsilon}(\Omega)$ for any $\varepsilon>0$. In particular, $\mathcal{A}$ belongs to $\mathcal{C}^{1}(\bar{\Omega})$ thanks to Sobolev imbeddings in two dimensions.

\subsection{Corner asymptotics}

In general, the magnetic potential $\mathcal{A}$ does not belong to $\mathcal{C}^{2}(\bar{\Omega})$, but the function $\mathcal{A}$ possesses a corner asymptotic expansion as the distance $r$ to $\mathbf{c}$ goes to zero.

In contrast with problems involving only homogeneous operators, problem (1) involves the lower order term $4 i \zeta^{2}$ in $\Omega_{-}$. As a consequence, according to the seminal paper of Kondratev [10], the singularities are not homogeneous functions, but infinite sums of quasi-homogeneous terms of the general form

$$
r^{\lambda+\ell} \log ^{n} r \Phi(\theta), \quad \lambda \in \mathbb{C}, \ell \in \mathbb{N}, n \in \mathbb{N}
$$

in polar coordinates $(r, \theta)$ centered at $\mathbf{c}$. In the present situation, the leading exponents $\lambda$ can be made precise: they are determined by the principal part of the operator at $\mathbf{c}$, which is nothing but the Laplacian $-\Delta$ at the interior point $\mathbf{c}$. Thus, the leading exponents are integers $k \in \mathbb{N}$ corresponding to leading singularities in the form of harmonic polynomials, written in polar coordinates as

$$
r^{k} \cos (k \theta-p \pi / 2), \quad k \in \mathbb{N}, p \in\{0,1\},
$$


- only $p=0$ is involved when $k=0$. This is why $\mathcal{A}$ can be expanded close to the corner as

$$
\mathcal{A}(r, \theta) \underset{r \rightarrow 0}{\sim} \wedge^{0,0} \mathfrak{S}^{0,0}(r, \theta)+\sum_{k \geqslant 1} \sum_{p \in\{0,1\}} \wedge^{k, p} \mathfrak{S}^{k, p}(r, \theta),
$$

where the terms $\mathfrak{S}^{k, p}$ are the so-called primal singular functions, which belong to the formal kernel of the considered operator in $\mathbb{R}^{2}$, and the numbers $\Lambda^{k, p}$ are the singular coefficients.

Therefore, the derivation of the singular functions $\mathfrak{S}^{k, p}$ and the determination of the singular coefficients $\Lambda^{k, p}$ are key points in understanding the behavior of the magnetic potential in the vicinity of the corner. This is the double aim of this work.

\subsection{Outline of the paper}

In this paper, we provide a constructive procedure to determine the singular functions $\mathfrak{S}^{k, p}$ as well as two different methods to compute the singular coefficients $\Lambda^{k, p}$. Generally speaking, the singular functions are sums of the kernel functions of the Laplacian plus shadow terms. Since the leading part of the operator is the Laplacian in the whole plane $\mathbb{R}^{2}$, its singular functions are the harmonic polynomials (3) and we show that for any $(k, p) \in \mathbb{N} \times\{0,1\}$, the singular function $\mathfrak{S}^{k, p}$ writes as formal series

$$
\mathfrak{S}^{k, p}(r, \theta)=r^{k} \sum_{j \geqslant 0}\left(i \zeta^{2}\right)^{j} r^{2 j} \sum_{n=0}^{j} \log ^{n} r \Phi_{j, n}^{k, p}(\theta),
$$

where the angular functions $\Phi_{j, n}^{k, p}$ are $\mathcal{C}^{1}$ functions of $\theta$ globally in $\mathbb{R} /(2 \pi \mathbb{Z})$ (and piecewise analytic).

The paper is organized as follows: In Section 2, we consider the case when $\zeta=0$ and show how the theory of corner expansions applies to the Taylor expansion of $\mathcal{A}$ in the interior point $\mathbf{c}$. For the determination of the coefficients $\Lambda^{k, p}$, we introduce the method of moments and the method of dual singular functions, which are both exact methods in this case. In Section 3 , for the case $\zeta \neq 0$, we provide the problem satisfied by the singular functions $\mathfrak{S}^{k, p}$ in the whole plane $\mathbb{R}^{2}$ split into two infinite sectors $\mathcal{S}_{ \pm}$(cf. Figure $1(\mathrm{~b})$ ). In order to determine the coefficients $\Lambda^{k, p}$, we generalize the method of moments, and we introduce the method of quasi-dual singular functions, after $[3,19]$. These methods are now approximate methods. We prove estimates for their convergence. In section 4 , we introduce a formalism using complex variables $z_{ \pm}$along the lines of [2], which simplifies the analytic calculations of the primal singular functions $\mathfrak{S}^{k, p}$ and the dual singular functions $\mathfrak{K}^{k, p}$, required by the quasi-dual function method. These dual singularities are composed of the kernel functions of the Laplacian with negative integer exponents, plus their shadows, quite similarly to the $\mathfrak{S}^{k, p}$. In this fourth section, only the first order shadows of the kernel functions of the Laplacian are given, while Appendix A provides tools of calculus of the shadows at any order. In the concluding Section 5, numerical simulations by finite elements methods illustrate the theoretical results.

\section{Laplace operator}

We first consider the case when $\zeta=0$, which means that we consider the solution $\mathcal{A}$ to the Laplace equation (2) with a smooth right-hand side $F$, whose support is located outside the interior point $\mathbf{c}$, and we look at the Taylor expansion of $\mathcal{A}$ at $\mathbf{c}$. Since $\mathcal{A}$ is harmonic in a neighborhood of $\mathbf{c}$, all the terms of its Taylor expansion are harmonic polynomials. A basis of harmonic polynomials can be written in polar coordinates as

$$
\mathfrak{s}^{k, p}(r, \theta)= \begin{cases}1, & \text { if } k=0 \text { and } p=0 \\ r^{k} \cos (k \theta-p \pi / 2), & \text { if } k \geqslant 1 \text { and } p=0,1\end{cases}
$$


and $\mathcal{A}$ admits the Taylor expansion

$$
\mathcal{A}(r, \theta) \underset{r \rightarrow 0}{\sim} \Lambda^{0,0} \mathfrak{s}^{0,0}(r, \theta)+\sum_{k \geqslant 1} \sum_{p \in\{0,1\}} \Lambda^{k, p} \mathfrak{s}^{k, p}(r, \theta) .
$$

There are several methods to extract the coefficients $\Lambda^{k, p}$. Besides taking point-wise values of partial derivatives (which would be unstable from the numerical approximation viewpoint), we can consider two methods:

1. The method of moments, which uses the orthogonality of the angular parts $\cos (k \theta-p \pi / 2)$ of the polynomials $\mathfrak{s}^{k, p}$.

2. The dual function method, which is the application to the present smooth case of a general method valid for corner coefficient extraction [14].

Both methods use non-polynomial dual harmonic functions singular at $r=0$. Let us set

$$
\mathfrak{k}^{k, p}(r, \theta)= \begin{cases}-\frac{1}{2 \pi} \log r, & \text { if } k=0, p=0, \\ \frac{1}{2 k \pi} r^{-k} \cos (k \theta-p \pi / 2), & \text { if } k \geqslant 1, p=0,1 .\end{cases}
$$

\subsection{The method of moments}

To extract the singular coefficients with the method of moments, we introduce the symmetric bilinear form $\mathcal{M}_{R}$ defined for $R>0$ by

$$
\mathcal{M}_{R}(K, A):=\frac{1}{R} \int_{r=R} K A R \mathrm{~d} \theta .
$$

We extract the scalar product of $\mathcal{A}$ versus 1 to compute $\Lambda^{0,0}$ and versus $\mathfrak{k}^{k, p}$ to compute $\Lambda^{k, p}$ when $k \geqslant 1$.

Proposition 2.1. Let $\mathcal{A}$ be the solution to the Laplace equation (2) with a smooth right-hand side $F$ with support outside the ball $\mathcal{B}(\mathbf{c}, R)$. Then

$$
\mathcal{M}_{R}(1, \mathcal{A})=2 \pi \wedge^{0,0}, \quad \text { and } \quad \mathcal{M}_{R}\left(\mathfrak{k}^{k, p}, \mathcal{A}\right)=\frac{1}{2 k} \wedge^{k, p}, \quad \text { for } k \geqslant 1, p=0,1 .
$$

Proof. The first equality is straightforward since

$$
\mathcal{M}_{R}\left(1, \mathfrak{s}^{l, p}\right)=0, \quad l \geqslant 1, p=0,1 .
$$

Using the orthogonality between the polynomials $\mathfrak{s}^{l, q}$ and the dual functions $\mathfrak{k}^{k, p}$ we infer

$$
\mathcal{M}_{R}\left(\mathfrak{k}^{k, p}, \mathfrak{s}^{l, q}\right)=\frac{1}{2 k} \delta_{l}^{k} \delta_{q}^{p}, \quad k \geqslant 1, l \geqslant 0, p, q=0,1,
$$

where $\delta_{i}^{j}$ denotes the Kronecker symbol. Then, Taylor expansion (6) of $\mathcal{A}$ leads to the second equality (9). 


\subsection{The method of dual functions}

Let us introduce the anti-symmetric bilinear form $\mathcal{J}_{R}$ defined over a circle of radius $R>0$ by

$$
\mathcal{J}_{R}(K, A):=\int_{r=R}\left(K \partial_{r} A-A \partial_{r} K\right) R \mathrm{~d} \theta
$$

Proposition 2.2. Let $\mathcal{A}$ be the solution to the Laplace equation (2) with a smooth right-hand side $F$, whose support is located outside the ball $\mathcal{B}(\mathbf{c}, R)$. Then

$$
\mathcal{J}_{R}\left(\mathfrak{k}^{0,0}, \mathcal{A}\right)=\Lambda^{0,0}, \quad \text { and } \quad \mathcal{J}_{R}\left(\mathfrak{k}^{k, p}, \mathcal{A}\right)=\Lambda^{k, p}, \quad \text { for } k \geqslant 1, p=0,1 .
$$

Proof. Since $\mathcal{J}_{R}\left(\mathfrak{k}^{0,0}, \mathfrak{s}^{k, p}\right)=0$ for $k \neq 0$, there holds

$$
\mathcal{J}_{R}\left(\mathfrak{k}^{0,0}, \mathcal{A}\right)=\mathcal{J}_{R}\left(\mathfrak{k}^{0,0}, \Lambda^{0,0}\right)=\Lambda^{0,0} .
$$

Straightforward computations lead to

$$
\mathcal{J}_{R}\left(\mathfrak{k}^{k, p}, \mathfrak{s}^{l, q}\right)=l \mathcal{M}_{R}\left(\mathfrak{k}^{k, p}, \mathfrak{s}^{l, q}\right)+k \mathcal{M}_{R}\left(\mathfrak{k}^{k, p}, \mathfrak{s}^{l, q}\right), \quad k, \quad l \geqslant 1, p, q=0,1 .
$$

Then, using (10) and (6), we obtain

$$
\mathcal{J}_{R}\left(\mathfrak{k}^{k, p}, \mathcal{A}\right)=\Lambda^{k, p}, \quad \text { for } k \geqslant 1, p=0,1,
$$

which ends the proof.

\section{The problem with angular conductor}

We now consider our problem of interest, i.e. problem ( 1 ) with $\zeta \neq 0$. Let $\omega \in(0,2 \pi)$ be the opening of the conductor part $\Omega_{-}$near its corner. We define the origin of the angular variable, so that $\theta=0$ cuts the conductor part by half (see Figure $1(\mathrm{~b})$ ). Let $\mathcal{S}_{+}$and $\mathcal{S}_{-}$be the two infinite sectors of $\mathbb{R}^{2}$ defined as

$$
\mathcal{S}_{-}=\left\{(x, y)=(r \cos \theta, r \sin \theta) \in \mathbb{R}^{2}: \quad r>0, \theta \in(-\omega / 2, \omega / 2)\right\}, \quad \mathcal{S}_{+}:=\mathbb{R}^{2} \backslash \overline{\mathcal{S}_{-}} .
$$

We denote by $\mathcal{G}$ the common boundary of $\mathcal{S}_{-}$and $\mathcal{S}_{+}$:

$$
\mathcal{G}=\partial \mathcal{S}_{-}=\partial \mathcal{S}_{+}=\left\{(x, y)=(r \cos \theta, r \sin \theta) \in \mathbb{R}^{2}: \quad r>0, \theta=-\frac{\omega}{2}, \frac{\omega}{2}\right\} .
$$

Finally, introduce the unit circle $\mathbb{T}=\mathbb{R} /(2 \pi \mathbb{Z})$ and set

$$
\mathbb{T}_{-}=(-\omega / 2, \omega / 2) \quad \text { and } \quad \mathbb{T}_{+}=\mathbb{T} \backslash \overline{\mathbb{T}_{-}} .
$$

Thus, the configuration $\left(\Omega_{-}, \Omega_{+}\right)$coincides with the configuration $\left(\mathcal{S}_{-}, \mathcal{S}_{+}\right)$inside a ball $\mathcal{B}(\mathbf{c}, R)$ for a sufficiently small $R$.

The positive parameter $\zeta$ being chosen, we denote by $\mathscr{L}_{\zeta}$ the operator defined on $\mathbb{R}^{2}$ by

$$
\mathscr{L}_{\zeta}(u)= \begin{cases}-\Delta u, & \text { in } \mathcal{S}_{+}, \\ -\Delta u+4 i \zeta^{2} u, & \text { in } \mathcal{S}_{-} .\end{cases}
$$

acting on functions $u$ such that $\left.\partial_{\theta}^{q} u_{-}\right|_{\mathcal{G}}=\left.\partial_{\theta}^{q} u_{+}\right|_{\mathcal{G}}$ for $q=0,1$. The singularities of problem (1) are these of the model operator $\mathscr{L}_{\zeta}$, with continuous Dirichlet and Neumann traces across $\mathcal{G}$. 
As described above, the asymptotics of $\mathcal{A}$ near the corner involve the singularities of $\mathscr{L}_{\zeta}$ which, by convention, are formal solutions $\mathfrak{U}$ to the equation $\mathscr{L}_{\zeta}(\mathfrak{U})=0$. It is therefore crucial to make explicit these singularities.

Note that the operator $\mathscr{L}_{\zeta}$ is the sum of its leading part $\mathscr{L}_{0}$ which is the Laplacian $-\Delta$ in $\mathbb{R}^{2}$, and of its secondary part $4 \mathrm{i} \zeta^{2} \mathscr{L}_{1}$ where $\mathscr{L}_{1}$ is the restriction to $\mathcal{S}_{-}$. According to the general principles of Kondratev's paper [10], the singularities $\mathfrak{U}$ of $\mathscr{L}_{\zeta}=\mathscr{L}_{0}+4 i \zeta^{2} \mathscr{L}_{1}$ can be described as formal sums

$$
\mathfrak{U}=\sum_{j}\left(i \zeta^{2}\right)^{j} \mathfrak{u}_{j}
$$

where each term $\mathfrak{u}_{j}$ is derived through an inductive process by solving recursively

$$
\mathscr{L}_{0} \mathfrak{u}_{0}=0, \quad \mathscr{L}_{0} \mathfrak{u}_{1}=-4 \mathscr{L}_{1} \mathfrak{u}_{0}, \quad \ldots, \quad \mathscr{L}_{0} \mathfrak{u}_{j}=-4 \mathscr{L}_{1} \mathfrak{u}_{j-1},
$$

in spaces of quasi-homogeneous functions $S^{\lambda}$ defined for $\lambda \in \mathbb{C}$ by

$$
\mathrm{S}^{\lambda}=\operatorname{Span}\left\{r^{\lambda} \log ^{q} r \Phi(\theta), \quad q \in \mathbb{N}, \quad \Phi \in \mathcal{C}^{1}(\mathbb{T}), \Phi^{ \pm} \in \mathcal{C}^{\infty}\left(\overline{\mathbb{T}}_{ \pm}\right)\right\}
$$

The numbers $\lambda$ are essentially determined by the leading equation $\mathscr{L}_{0} \mathfrak{u}_{0}=0$, whose solutions are harmonic functions in $\mathbb{R}^{2} \backslash\{\mathbf{c}\}$. The first term $\mathfrak{u}_{0}$ is the leading part of the singularity while the next terms $\mathfrak{u}_{j}$, for $j \geqslant 1$, are called the shadows.

The existence of the terms $\mathfrak{u}_{j}$ relies on the following result.

Lemma 3.1. For $\lambda \in \mathbb{C}$, let $\mathrm{S}^{\lambda}$ be defined by (16) and let $\mathrm{T}^{\lambda}$ be defined as

$$
\mathrm{T}^{\lambda}=\operatorname{Span}\left\{r^{\lambda} \log ^{q} r \Psi(\theta), \quad q \in \mathbb{N}, \quad \Psi \in L^{2}(\mathbb{T}), \quad \Psi^{ \pm} \in \mathcal{C}^{\infty}\left(\overline{\mathbb{T}}_{ \pm}\right)\right\}
$$

For an element $\mathfrak{g}$ of $\mathrm{S}^{\lambda}$ or $\mathrm{T}^{\lambda}$, the degree deg $\mathfrak{g}$ of $\mathfrak{g}$ is its degree as polynomial of $\log r$.

Let $\lambda \in \mathbb{C}$ and $\mathfrak{f} \in \mathrm{T}^{\lambda-2}$. Then, there exists $\mathfrak{u} \in \mathrm{S}^{\lambda}$ such that $\mathscr{L}_{0} \mathfrak{u}=\mathfrak{f}$. Moreover

(i) If $\lambda \notin \mathbb{Z}, \operatorname{deg} \mathfrak{u}=\operatorname{deg} \mathfrak{f}$,

(ii) If $\lambda \in \mathbb{Z} \backslash\{0\}, \operatorname{deg} \mathfrak{u} \leqslant \operatorname{deg} \mathfrak{f}+1$,

(iii) If $\lambda=0$, $\operatorname{deg} \mathfrak{u} \leqslant \operatorname{deg} \mathfrak{f}+2$.

Proof. We follow the technique of [5, Ch. 4]. We introduce the holomorphic family of operators $\mathscr{M}$ (also called Mellin symbol) associated with $\mathscr{L}_{0}$ in polar coordinates

$$
\mathscr{L}_{0}=-\Delta=-r^{-2}\left(\left(r \partial_{r}\right)^{2}+\partial_{\theta}^{2}\right) \quad \text { and } \quad \mathscr{M}(\mu)=-\left(\mu^{2}+\partial_{\theta}^{2}\right), \theta \in \mathbb{T} \text {. }
$$

Note the following facts

i) The poles of $\mathscr{M}(\mu)^{-1}$ are the integers,

ii) If $k$ is a nonzero integer, the pole of $\mathscr{M}(\mu)^{-1}$ in $k$ is of order 1 ,

iii) The pole of $\mathscr{M}(\mu)^{-1}$ in 0 is of order 2 .

Let $\mathfrak{f} \in T^{\lambda-2}$. Then

$$
\mathfrak{f}=\sum_{q=0}^{\operatorname{deg} \mathfrak{f}} r^{\lambda-2} \log ^{q} r \Psi_{q}(\theta) .
$$

$R R n^{\circ} 8204$ 
We have the residue formula ${ }^{1}$

$$
\mathfrak{f}=\operatorname{Res}_{\mu=\lambda}\left\{r^{\mu-2} \sum_{q=0}^{\operatorname{deg} \mathfrak{f}} \frac{q ! \Psi_{q}}{(\mu-\lambda)^{q+1}}\right\}
$$

Setting

$$
\mathfrak{u}=\operatorname{Res}_{\mu=\lambda}\left\{r^{\mu} \mathscr{M}(\mu)^{-1} \sum_{q=0}^{\operatorname{deg} \mathfrak{f}} \frac{q ! \Psi_{q}}{(\mu-\lambda)^{q+1}}\right\}
$$

we check that $\mathscr{L}_{0} \mathfrak{u}=\mathfrak{f}$ and the assertions on deg $\mathfrak{u}$ are consequences of the above properties i), ii) and iii).

At this point, we distinguish the primal singular functions, which belong to $H^{1}$ in any bounded neighborhood $\mathcal{B}$ of $\mathbf{c}$, from the dual singular functions, which do not belong to $H^{1}$ in such a neighborhood. The primal singular functions $\mathfrak{S}$ appear in the expansion as $r \rightarrow 0$ of the solutions to problem (1), while the dual singular functions $\mathfrak{K}$ are needed for the determination of the coefficients $\Lambda$ involved in the asymptotics.

\subsection{Principles of the construction of the primal singularities}

The primal singular functions start with (quasi-homogeneous) terms $\mathfrak{u}_{0} \in H^{1}(\mathcal{B})$ satisfying $\Delta \mathfrak{u}_{0}=0$. Therefore, $\mathfrak{u}_{0}$ is a homogeneous harmonic polynomial. We are looking for a basis for primal singular functions, so we choose $\mathfrak{u}_{0}=\mathfrak{s}^{k, p}$ (for $(k, p)=(0,0)$ and for any $(k, p) \in \mathbb{N}^{*} \times\{0,1\}$ ) according to notation (5). In order to have coherent notation, we set $\mathfrak{s}_{0}^{k, p}:=\mathfrak{s}^{k, p}$. Each $\mathfrak{s}_{0}^{k, p}$ belongs to $\boldsymbol{S}^{k}$ and is the leading part of the singular function $\mathfrak{S}^{k, p}$ defined by

$$
\mathfrak{S}^{k, p}(r, \theta)=\sum_{j \geqslant 0}\left(i \zeta^{2}\right)^{j} \mathfrak{s}_{j}^{k, p}(r, \theta)
$$

For $j \geqslant 1$, the terms $\mathfrak{s}_{j}^{k, p}$ in the previous series are the shadow terms of order $j$. According to the sequence of problems (15), the function $\mathfrak{s}_{j}^{k, p}$ is searched in $S^{k+2 j}$ as a particular solution to the following problem:

$$
\left\{\begin{array}{l}
\Delta \mathfrak{s}_{j}^{k, p+}=0, \quad \text { in } \mathcal{S}_{+}, \\
\Delta \mathfrak{s}_{j}^{k, p-}=4 \mathfrak{s}_{j-1}^{k, p-}, \quad \text { in } \mathcal{S}_{-} .
\end{array}\right.
$$

Belonging to $\mathrm{S}^{k+2 j}$ includes the following transmission conditions on $\mathcal{G}$ :

$$
\left\{\begin{array}{l}
\left.\mathfrak{s}_{j}^{k, p+}\right|_{\mathcal{G}}=\left.\mathfrak{s}_{j}^{k, p-}\right|_{\mathcal{G}} \\
\left.\partial_{\theta} \mathfrak{s}_{j}^{k, p+}\right|_{\mathcal{G}}=\left.\partial_{\theta} \mathfrak{s}_{j}^{k, p-}\right|_{\mathcal{G}} .
\end{array}\right.
$$

Lemma 3.2. Let $(k, p)=(0,0)$ or $(k, p) \in \mathbb{N}^{*} \times\{0,1\}$. Let $\mathfrak{s}_{0}^{k, p}$ be defined as

$$
\mathfrak{s}_{0}^{k, p}(r, \theta)= \begin{cases}1, & \text { if } k=0 \text { and } p=0, \\ r^{k} \cos (k \theta-p \pi / 2), & \text { if } k \geqslant 1 \text { and } p=0,1 .\end{cases}
$$

Then, for any $j \geqslant 1$, there exists $\mathfrak{s}_{j}^{k, p} \in \mathrm{S}^{k+2 j}$ satisfying (19)-(20). Moreover $\operatorname{deg} \mathfrak{s}_{j}^{k, p} \leqslant j$. When $p=0$, we choose $\mathfrak{s}_{j}^{k, p}$ as an even function ${ }^{2}$ of $\theta$ and when $p=1, \mathfrak{s}_{j}^{k, p}$ is chosen to be odd.

\footnotetext{
${ }^{1}$ For a meromorphic function $f$ of the complex variable $\mu$ with pole at $\lambda$ and for any simple contour $\mathcal{C}$ surrounding $\lambda$ and no other pole of $f$, there holds $\underset{\mu=\lambda}{\operatorname{Res}}\{f(\mu)\}=\frac{1}{2 i \pi} \int_{\mathcal{C}} f(\mu) \mathrm{d} \mu$.

${ }^{2}$ We understand that $u$ is an even function of $\theta$ if $u(-\theta)=u(\theta)$ for all $\theta \in \mathbb{R} /(2 \pi \mathbb{Z})$.
} 
Proof. We apply recursively Lemma 3.1. We start with $\mathfrak{s}_{0}^{k, p}$, which belongs to $\mathrm{S}^{k}$ and satisfies deg $\mathfrak{s}_{0}^{k, p}=$ 0 . The right-hand side of (19) for $j=1$ is $4 \mathfrak{s}_{0}^{k, p} \mathbb{1}_{\mathcal{S}_{-}}$. It is discontinuous across $\mathcal{G}$, belongs to $\mathrm{T}^{k}$, and is even (resp. odd) for $p=0$ (resp. $p=1$ ). By Lemma 3.1 there exists $\mathfrak{s}_{1}^{k+2, p} \in \mathrm{S}^{k+2}$ solution to (19)-(20) for $j=1$, and $\operatorname{deg} \mathfrak{s}_{1}^{k+2} \leqslant 1$. Since $\Delta$ commutes with the symmetry with respect to $x$ axis, there exists a particular solution with the same parity as the right-hand side. The proof for any $j$ follows from a recursion argument.

These definitions being set, we can write the expansion of the solution to problem (1) as

$$
\mathcal{A}(r, \theta) \underset{r \rightarrow 0}{\sim} \wedge^{0,0} \mathfrak{S}^{0,0}(r, \theta)+\sum_{k \geqslant 1} \sum_{p \in\{0,1\}} \wedge^{k, p} \mathfrak{S}^{k, p}(r, \theta) .
$$

Moreover, using the results of Lemma 3.2 and (16) in (18), it is straightforward that for any $(k, p) \in$ $\mathbb{N}^{*} \times\{0,1\}$ or $(k, p)=(0,0)$, the singular function $\mathfrak{S}^{k, p}$ writes as formal series

$$
\mathfrak{S}^{k, p}(r, \theta)=r^{k} \sum_{j \geqslant 0}\left(i \zeta^{2}\right)^{j} r^{2 j} \sum_{n=0}^{j} \log ^{n} r \Phi_{j, n}^{k, p}(\theta)
$$

where the angular functions $\Phi_{j, n}^{k, p}$ are $\mathcal{C}^{1}$ functions of $\theta$ globally in $\mathbb{R} /(2 \pi \mathbb{Z})$ (and piecewise analytic).

\subsection{Principles of the construction of the dual singularities}

We start with the dual singularities of the interior Laplace operator introduced in (7). Setting $\mathfrak{k}_{0}^{k, p}=$ $\mathfrak{k}^{k, p}$, the dual singularities of $\mathscr{L}_{\zeta}$ are given by series

$$
\mathfrak{K}^{k, p}(r, \theta)=\sum_{j \geqslant 0}\left(i \zeta^{2}\right)^{j \mathfrak{k}_{j}^{k, p}}(r, \theta),
$$

where, for $j \geqslant 1$, the shadow terms $\mathfrak{k}_{j}^{k, p}$ are solutions in $\mathbf{S}^{-k+2 j}$ to

$$
\left\{\begin{array}{l}
\Delta \mathfrak{k}_{j}^{k, p+}=0, \quad \text { in } \mathcal{S}_{+}, \\
\Delta \mathfrak{k}_{j}^{k, p-}=4 \mathfrak{k}_{j-1}^{k, p-}, \quad \text { in } \mathcal{S}_{-},
\end{array}\right.
$$

with the same transmission conditions as (20). We deduce from Lemma 3.1:

Lemma 3.3. Let $(k, p)=(0,0)$ or $(k, p) \in \mathbb{N}^{*} \times\{0,1\}$. Let $\mathfrak{k}_{0}^{k, p}$ be defined as

$$
\mathfrak{k}_{0}^{k, p}(r, \theta)= \begin{cases}-\frac{1}{2 \pi} \log r, & \text { if } k=0, p=0, \\ \frac{1}{2 k \pi} r^{-k} \cos (k \theta-p \pi / 2), & \text { if } k \geqslant 1, p=0,1 .\end{cases}
$$

Then, for any $j \geqslant 1$, there exists $\mathfrak{k}_{j}^{k, p} \in \mathrm{S}^{-k+2 j}$ satisfying (24). Moreover, if $j$ is odd, or if $j$ is even and $2 j<k$, then $\operatorname{deg} \mathfrak{k}_{j}^{k, p} \leqslant j$. Otherwise (i.e. if $j$ is even and $2 j \geqslant k$ ) then $\operatorname{deg} \mathfrak{k}_{j}^{k, p} \leqslant j+1$. When $p=0$, we choose $\mathfrak{k}_{j}^{k, p}$ as an even function of $\theta$ and when $p=1, \mathfrak{k}_{j}^{k, p}$ is chosen to be odd. 
Using the results of Lemma 3.3 and (16) in (23), it is straightforward that for any $(k, p) \in$ $\mathbb{N}^{*} \times\{0,1\}$ or $(k, p)=(0,0)$, the singular function $\mathfrak{K}^{k, p}$ writes as formal series

$$
\mathfrak{K}^{k, p}(r, \theta)= \begin{cases}r^{-k} \sum_{j \geqslant 0}\left(i \zeta^{2}\right)^{j} r^{2 j} \sum_{n=0}^{j} \log ^{n} r \Psi_{j, n}^{k, p}(\theta) & \text { (k odd), } \\ r^{-k}\left(\sum_{0 \leqslant j<k / 2}\left(i \zeta^{2}\right)^{j} r^{2 j} \sum_{n=0}^{j} \log ^{n} r \Psi_{j, n}^{k, p}(\theta)+\sum_{j \geqslant k / 2}\left(i \zeta^{2}\right)^{j} r^{2 j} \sum_{n=0}^{j+1} \log ^{n} r \Psi_{j, n}^{k, p}(\theta)\right) & (k \text { even }) .\end{cases}
$$

where the angular functions $\Psi_{j, n}^{k, p}$ are $\mathcal{C}^{1}$ functions of $\theta$ globally in $\mathbb{R} /(2 \pi \mathbb{Z})$ (and piecewise analytic).

\subsection{A first try to extract the singular coefficients: the method of moments}

A naive approach to extract the coefficients consists in following the heuristics of the case when $\zeta=0$, cf. (8)-(9): compute $\mathcal{M}_{R}(1 /(2 \pi), \mathcal{A})$ in order to extract the coefficient $\Lambda^{0,0}$ and $\mathcal{M}_{R}\left(2 k k^{k, p}, \mathcal{A}\right)$ for the coefficient $\Lambda^{k, p}$ of expansion (21). This method makes possible a quite rough approximation of the first coefficients only, as described below. For a systematic computation of all the coefficients, the method of quasi-dual functions, described in the next section is more accurate.

Let us set

$$
M_{R}^{k, p}(\mathcal{A})= \begin{cases}\frac{1}{2 \pi} \mathcal{M}_{R}(1, \mathcal{A}) & \text { if } k=0 \text { and } p=0, \\ 2 k \mathcal{M}_{R}\left(\mathfrak{k}_{0}^{k, p}, \mathcal{A}\right) & \text { if } k \geqslant 1 \text { and } p=0,1 .\end{cases}
$$

We input the expansion (21) of $\mathcal{A}$ in $M_{R}^{k, p}$ to obtain the formal expression

$$
\underline{M}_{R}(\mathcal{A})=\underline{\mathcal{M}_{R}} \underline{\Lambda}(\mathcal{A}),
$$

with $\underline{M}_{R}(\mathcal{A})$ and $\underline{\Lambda}(\mathcal{A})$ the column vectors $\left(M_{R}^{0,0}(\mathcal{A}), M_{R}^{1,0}(\mathcal{A}), M_{R}^{1,1}(\mathcal{A}), \ldots\right)^{\top}$ and $\left(\wedge^{0,0}, \wedge^{1,0}, \wedge^{1,1}, \ldots\right)^{\top}$, respectively, and $\underline{\mathcal{M}}_{R}$ the matrix with coefficients

$$
\mathcal{M}_{R}^{k, p ; k^{\prime}, p^{\prime}}=M_{R}^{k, p}\left(\mathfrak{S}^{k^{\prime}, p^{\prime}}\right)
$$

Hence, we deduce the formal expression of the coefficients $\wedge^{k, p}$

$$
\underline{\Lambda}(\mathcal{A})=\left(\underline{\mathcal{M}}_{R}\right)^{-1} \underline{M}_{R}(\mathcal{A})
$$

In order to give a sense to this expression, and to design a method for the determination of the coefficients $\Lambda^{k, p}$, we have to truncate the matrix $\underline{\mathcal{M}}_{R}$ by taking its first $N$ rows and columns, $N=$ $1,2, \ldots$

Let us denote for shortness

$$
R_{0}=\zeta R(1+\sqrt{|\log R|})
$$

and assume that $R \leqslant 1$. Note that, relying on Proposition 2.1, Lemma 3.2 and formula (18), we find

$$
\mathcal{M}_{R}^{k, p ; k, p} \underset{R \rightarrow 0}{=} 1+\mathcal{O}\left(R_{0}^{2}\right) \quad \text { and } \quad \mathcal{M}_{R}^{k, p ; k^{\prime}, p^{\prime}}=0 \text { if } p \neq p^{\prime}
$$

We also immediately check that

$$
\mathcal{M}_{R}^{k, p ; k^{\prime}, p^{\prime}} \underset{R \rightarrow 0}{=} \mathcal{O}\left(R^{-k+k^{\prime}} R_{0}^{2}\right) \quad \text { if } k \neq k^{\prime} .
$$


Let us emphasize the blow up of this coefficient as $R \rightarrow 0$ if $k \geqslant k^{\prime}+2$, which prevents the use of this method to approximate the singular coefficients at any level of accuracy. This drawback is avoided by the method of the quasi-dual functions presented in the next subsection.

However, due to its simplicity, the method of moments can be useful if a low order of accuracy of the first 3 coefficients is sufficient. In the following, we list what can be deduced from the consideration of the lowest values for the cut-off dimension $N$.

- $N=1$. We consider the sole approximate equation

$$
M_{R}^{0,0}(\mathcal{A})=M_{R}^{0,0}\left(\mathfrak{S}^{0,0}\right) \wedge^{0,0}+\mathcal{O}\left(R R_{0}^{2}\right) .
$$

The remainder $\mathcal{O}\left(R R_{0}^{2}\right)$ corresponds to the neglected terms in the asymptotics of $\mathcal{A}$. Using Lemma 3.2 for the structure of $\mathfrak{S}^{0,0}$, we find

$$
\mathfrak{S}^{0,0}(r, \theta) \underset{r \rightarrow 0}{=} 1+\mathrm{i}(\zeta r)^{2} \sum_{q=0}^{1} \log ^{q} r \Phi_{1, q}^{0,0}(\theta)+\mathcal{O}\left((\zeta r)^{4} \log ^{2} r\right)
$$

Therefore we deduce

i) Using only the first term in the asymptotics of $\mathfrak{S}^{0,0}$, we find

$$
\Lambda^{0,0} \underset{R \rightarrow 0}{=} M_{R}^{0,0}(\mathcal{A})+\mathcal{O}\left(R_{0}^{2}\right) .
$$

ii) If we compute $\frac{1}{2 \pi} \int_{\mathbb{T}} \Phi_{1, q}^{0,0}(\theta) d \theta=: m_{1, q}^{0,0 ; 0,0}$, we find

$$
\Lambda^{0,0} \underset{R \rightarrow 0}{=} \frac{M_{R}^{0,0}(\mathcal{A})}{1+\mathrm{i}(\zeta R)^{2} \sum_{q=0}^{1} m_{1, q}^{0,0 ; 0,0} \log ^{q} R}+\mathcal{O}\left(R R_{0}^{2}\right)+\mathcal{O}\left(R_{0}^{4}\right) .
$$

iii) If we compute more terms in the asymptotics of $\mathfrak{S}^{0,0}$, we improve the piece of error $\mathcal{O}\left(R_{0}^{4}\right)$, but leave the piece $\mathcal{O}\left(R R_{0}^{2}\right)$ unchanged.

- $N=3$. We consider the 3 approximate equations concerning $M_{R}^{0,0}(\mathcal{A}), M_{R}^{1,0}(\mathcal{A})$ and $M_{R}^{1,1}(\mathcal{A})$. For parity reasons, we have a $2 \times 2$ system

$$
\begin{aligned}
& M_{R}^{0,0}(\mathcal{A})=M_{R}^{0,0}\left(\mathfrak{S}^{0,0}\right) \wedge^{0,0}+M_{R}^{0,0}\left(\mathfrak{S}^{1,0}\right) \wedge^{1,0}+\mathcal{O}\left(R^{2} R_{0}^{2}\right), \\
& M_{R}^{1,0}(\mathcal{A})=M_{R}^{1,0}\left(\mathfrak{S}^{0,0}\right) \wedge^{0,0}+M_{R}^{1,0}\left(\mathfrak{S}^{1,0}\right) \wedge^{1,0}+\mathcal{O}\left(R R_{0}^{2}\right),
\end{aligned}
$$

and an independent equation

$$
M_{R}^{1,1}(\mathcal{A})=M_{R}^{1,1}\left(\mathfrak{S}^{1,1}\right) \Lambda^{1,1}+\mathcal{O}\left(R R_{0}^{2}\right)
$$

For this latter equation, we have a similar analysis as for $\Lambda^{0,0}$ alone $(N=1)$ : Using again Lemma 3.2 for the structure of $\mathfrak{S}^{1,1}$, we find

$$
\mathfrak{S}^{1,1}(r, \theta) \underset{r \rightarrow 0}{=} r \sin \theta+\mathrm{i}(\zeta r)^{2} r \sum_{q=0}^{1} \log ^{q} r \Phi_{1, q}^{1,1}(\theta)+\mathcal{O}\left((\zeta r)^{4} r \log ^{2} r\right),
$$

and with the first term in the asymptotics of $\mathfrak{S}^{1,1}$, we obtain

$$
\Lambda^{1,1} \underset{R \rightarrow 0}{=} M_{R}^{1,1}(\mathcal{A})+\mathcal{O}\left(R_{0}^{2}\right)
$$

RR n ${ }^{\circ} 8204$ 
whereas, using two terms in the asymptotics of $\mathfrak{S}^{1,1}$, and computing $\frac{1}{2 \pi} \int_{\mathbb{T}} \sin \theta \Phi_{1, q}^{1,1}(\theta) \mathrm{d} \theta=: m_{1, q}^{1,1 ; 1,1}$, we find

$$
\Lambda^{1,1} \underset{R \rightarrow 0}{=} \frac{M_{R}^{1,1}(\mathcal{A})}{1+\mathrm{i}(\zeta R)^{2} \sum_{q=0}^{1} m_{1, q}^{1,1 ; 1,1} \log ^{q} R}+\mathcal{O}\left(R R_{0}^{2}\right)+\mathcal{O}\left(R_{0}^{4}\right) .
$$

For the $2 \times 2$ system, according to the number of terms considered in the asymptotics (18) of $\mathfrak{S}^{0,0}$ and $\mathfrak{S}^{1,0}$ we find the following formulas.

i) If we take one term for $\mathfrak{S}^{0,0}$ in (27) and one term for $\mathfrak{S}^{1,0}$ in

$$
\mathfrak{S}^{1,0}(r, \theta) \underset{r \rightarrow 0}{=} r \cos \theta+\mathcal{O}\left((\zeta r)^{2} r \log ^{2} r\right)
$$

we obtain a diagonal system and

$$
\Lambda^{0,0} \underset{R \rightarrow 0}{=} M_{R}^{0,0}(\mathcal{A})+\mathcal{O}\left(R_{0}^{2}\right) \quad \text { and } \quad \Lambda^{1,0} \underset{R \rightarrow 0}{=} M_{R}^{1,0}(\mathcal{A})+\mathcal{O}\left(R^{-1} R_{0}^{2}\right) .
$$

ii) If we take two terms for $\mathfrak{S}^{0,0}$ and one for $\mathfrak{S}^{1,0}$, we obtain (28) again, and computing $m_{1, q}^{1,0 ; 0,0}$ as $\frac{1}{\pi} \int_{\mathbb{T}} \cos \theta \Phi_{1, q}^{0,0}(\theta) \mathrm{d} \theta$, we find

$$
\Lambda^{1,0} \underset{R \rightarrow 0}{=} M_{R}^{1,0}(\mathcal{A})-M_{R}^{0,0}(\mathcal{A}) \frac{\mathrm{i} R^{-1}(\zeta R)^{2} \sum_{q=0}^{1} m_{1, q}^{1,0 ; 0,0} \log ^{q} R}{1+\mathrm{i}(\zeta R)^{2} \sum_{q=0}^{1} m_{1, q}^{0,0 ; 0,0} \log ^{q} R}+\mathcal{O}\left(R R_{0}^{2}\right)+\mathcal{O}\left(R^{-1} R_{0}^{4}\right) .
$$

iii) Taking more terms, we improve some parts of the remainder, we still have $\mathcal{O}\left(R^{2} R_{0}^{2}\right)$ for $\Lambda^{0,0}$, and $\mathcal{O}\left(R R_{0}^{2}\right)$ for $\Lambda^{1,0}$, but we always have a term containing $R^{-1}$, preventing the good accuracy of the method.

\subsection{Extraction of singular coefficients by the method of quasi-dual functions}

When $\zeta \neq 0$, and in contrast to the case $\zeta=0$, we do not use exact dual functions satisfying $\mathscr{L}_{\zeta} \mathfrak{K}=0$ inside the anti-symmetric bilinear form $\mathcal{J}_{R}(11)$. Instead we use the quasi-dual functions $\mathfrak{K}_{m}^{k, p}$, which are the truncated series of (23):

$$
\mathfrak{K}_{m}^{k, p}(r, \theta):=\sum_{j=0}^{m}\left(i \zeta^{2}\right)^{j} \mathfrak{k}_{j}^{k, p}(r, \theta) .
$$

Here, $m$ is a nonnegative integer, which is the order of the quasi-dual function. By construction

$$
\mathscr{L}_{\zeta} \mathfrak{K}_{m}^{k, p}=4 i \zeta^{2}\left(i \zeta^{2}\right)^{m} \mathfrak{k}_{m}^{k, p} \mathbb{1}_{\mathcal{S}_{-}},
$$

which is not zero, but smaller and smaller as $r \rightarrow 0$ when $m$ is increased. The extraction of coefficients $\wedge^{k, p}$ in expansion (21) is performed through the evaluation of quantities

$$
\mathcal{J}_{R}\left(\mathfrak{K}_{m}^{k, p}, \mathcal{A}\right), \quad k=0,1,2, \ldots
$$

and corresponding $p \in\{0,1\}$, for suitable values of $m \in\{0,1, \ldots\}$. The quasi-dual function method was introduced in [3] for straight edges and developed in [19] for circular edges and homogeneous operators with constant coefficients. The expansions considered there do not contain any logarithmic terms. Here, we revisit this theory in our framework where, on the contrary, we have an accumulation of logarithmic terms. The main result follows. 
Theorem 3.4. Let $\mathcal{A}$ be the solution to problem (1), under the assumptions of the introduction. Let $k \in \mathbb{N}$ and $p \in\{0,1\}$ ( $p=0$ if $k=0)$. Let $m$ such that $2 m+2>k$. For the extraction quantity $\mathcal{J}_{R}\left(\mathfrak{K}_{m}^{k, p}, \mathcal{A}\right)$ defined through (11) and (33), there exist coefficients $\mathcal{J}^{k, p ; k^{\prime}, p^{\prime}}$ independent of $R$ and $\mathcal{A}$ such that

$$
\mathcal{J}_{R}\left(\mathfrak{K}_{m}^{k, p}, \mathcal{A}\right) \underset{R \rightarrow 0}{=} \Lambda^{k, p}+\sum_{\ell=1}^{[k / 2]} \mathcal{J}^{k, p ; k-2 \ell, p} \Lambda^{k-2 \ell, p}+\mathcal{O}\left(R^{-k} R_{0}^{2 m+2} \log R\right),
$$

where $R_{0}$ is defined in (26). If $p=1$, the remainder is improved to $\mathcal{O}\left(R^{1-k} R_{0}^{2 m+2} \log R\right)$. The extra term $\log R$ disappears if $k$ is odd.

Remark 3.5. The collection of equations (35) for $p \in\{0,1\}$, and $k$ belonging to $\{0,2, \ldots, 2 L\}$ or to $\{1,3, \ldots, 2 L+1\}$, with $L \in \mathbb{N}$, forms a lower triangular system with invertible diagonal.

Example 3.6. i) We have the following simple formulas for $m=0$ :

- For $k=0$

$$
\Lambda^{0,0} \underset{R \rightarrow 0}{=} \mathcal{J}_{R}\left(\mathfrak{K}_{0}^{0,0}, \mathcal{A}\right)+\mathcal{O}\left(R_{0}^{2} \log R\right)
$$

- For $k=1$

$$
\Lambda^{1,0} \underset{R \rightarrow 0}{=} \mathcal{J}_{R}\left(\mathfrak{K}_{0}^{1,0}, \mathcal{A}\right)+\mathcal{O}\left(R^{-1} R_{0}^{2}\right) \quad \text { and } \quad \Lambda^{1,1} \underset{R \rightarrow 0}{=} \mathcal{J}_{R}\left(\mathfrak{K}_{0}^{1,1}, \mathcal{A}\right)+\mathcal{O}\left(R_{0}^{2}\right)
$$

If we know $m$ more terms in the quasi-dual functions, then $\mathcal{O}\left(R_{0}^{2}\right)$ is replaced by $\mathcal{O}\left(R_{0}^{2+2 m}\right)$ in the remainder terms.

ii) For $k=2$, we need $m \geqslant 1$. We have

$$
\Lambda^{2,1} \underset{R \rightarrow 0}{=} \mathcal{J}_{R}\left(\mathfrak{K}_{m}^{2,1}, \mathcal{A}\right)+\mathcal{O}\left(R^{-1} R_{0}^{2+2 m} \log R\right),
$$

for the odd singularity, and

$$
\Lambda^{2,0} \underset{R \rightarrow 0}{=} \mathcal{J}_{R}\left(\mathfrak{K}_{m}^{2,0}, \mathcal{A}\right)-\mathcal{J}^{2,0 ; 0,0} \Lambda^{0,0}+\mathcal{O}\left(R^{-2} R_{0}^{2+2 m} \log R\right),
$$

for the even singularity. An explicit formula for the coefficient $\mathcal{J}^{2,0 ; 0,0}$ is given later on, see (65), as a particular case of the general formula (44).

Proof of Theorem 3.4. Introduce the truncated series of singularities (18):

$$
\mathfrak{S}_{m}^{k, p}(r, \theta):=\sum_{j=0}^{m}\left(i \zeta^{2}\right)^{j} \mathfrak{s}_{j}^{k, p}(r, \theta)
$$

We have

$$
\mathscr{L}_{\zeta} \mathfrak{S}_{m}^{k, p}=4 i \zeta^{2}\left(i \zeta^{2}\right)^{m} \mathfrak{s}_{m}^{k, p} \mathbb{1}_{\mathcal{S}_{-}} .
$$

In order to prove formula (35), let us evaluate $\mathcal{J}_{R}\left(\mathfrak{K}_{m}^{k, p}, \mathfrak{S}_{m^{\prime}}^{k^{\prime}, p^{\prime}}\right)$ with any chosen $k^{\prime}, p^{\prime}$ and $m^{\prime} \geqslant m$. For parity reasons, $\mathcal{J}_{R}\left(\mathfrak{K}_{m}^{k, p}, \mathfrak{S}_{m^{\prime}}^{k^{\prime}, p^{\prime}}\right)$ is zero if $p \neq p^{\prime}$. Therefore, from now on we take $p^{\prime}=p$.

For $\varepsilon \in(0, R)$, denote by $\mathcal{C}(\varepsilon, R)$ be the annulus $\{(x, y): r \in(\varepsilon, R)\}$. Thanks to Green formula the following equality holds:

$$
\mathcal{J}_{R}\left(\mathfrak{K}_{m}^{k, p}, \mathfrak{S}_{m^{\prime}}^{k^{\prime}, p}\right)-\mathcal{J}_{\varepsilon}\left(\mathfrak{K}_{m}^{k, p}, \mathfrak{S}_{m^{\prime}}^{k^{\prime}, p}\right)=\int_{\mathcal{C}(\varepsilon, R)}\left(\mathfrak{K}_{m}^{k, p} \mathscr{L}_{\zeta} \mathfrak{S}_{m^{\prime}}^{k^{\prime}, p}-\mathscr{L}_{\zeta} \mathfrak{K}_{m}^{k, p} \mathfrak{S}_{m^{\prime}}^{k^{\prime}, p}\right) r \mathrm{~d} r \mathrm{~d} \theta
$$

RR n ${ }^{\circ} 8204$ 
hence, by formulas (34) and (36), we deduce

$$
\mathcal{J}_{R}\left(\mathfrak{K}_{m}^{k, p}, \mathfrak{S}_{m^{\prime}}^{k^{\prime}, p^{\prime}}\right)-\mathcal{J}_{\varepsilon}\left(\mathfrak{K}_{m}^{k, p}, \mathfrak{S}_{m^{\prime}}^{k^{\prime}, p}\right)=4 i \zeta^{2} \int_{\mathcal{C}(\varepsilon, R)}\left(\mathfrak{K}_{m}^{k, p}\left(i \zeta^{2}\right)^{m^{\prime}} \mathfrak{s}_{m^{\prime}}^{k^{\prime}, p}-\left(i \zeta^{2}\right)^{m_{\mathfrak{k}^{\prime}}^{k, p}} \mathfrak{S}_{m^{\prime}}^{k^{\prime}, p}\right) \mathbb{1}_{\mathcal{S}_{-}} r \mathrm{~d} r \mathrm{~d} \theta .
$$

Using Lemmas 3.2 and 3.3, and taking the condition $-k+2 m+2>0$ into account, we find

$$
\mathcal{J}_{R}\left(\mathfrak{K}_{m}^{k, p}, \mathfrak{S}_{m^{\prime}}^{k^{\prime}, p}\right)-\mathcal{J}_{\varepsilon}\left(\mathfrak{K}_{m}^{k, p}, \mathfrak{S}_{m^{\prime}}^{k^{\prime}, p}\right) \underset{R \rightarrow 0}{=} \mathcal{O}\left(R^{-k+k^{\prime}} R_{0}^{2 m^{\prime}+2}\right)+\mathcal{O}\left(R^{-k+k^{\prime}} R_{0}^{2 m+2} \log R\right),
$$

independently of $\varepsilon<R$, - the term $\log R$ can be omitted if $k$ is odd. Hence, since $m^{\prime} \geqslant m$ it yields:

$$
\begin{aligned}
\mathcal{J}_{R}\left(\mathfrak{K}_{m}^{k, p}, \mathfrak{S}_{m^{\prime}}^{k^{\prime}, p}\right)-\mathcal{J}_{\varepsilon}\left(\mathfrak{K}_{m}^{k, p}, \mathfrak{S}_{m^{\prime}}^{k^{\prime}, p}\right) & \underset{R \rightarrow 0}{=} \mathcal{O}\left(R^{-k+k^{\prime}} R_{0}^{2 m+2} \log R\right) \\
& =\zeta^{2 m+2} \mathcal{O}\left(R^{-k+k^{\prime}+2 m+2} \log ^{m+2} R\right) .
\end{aligned}
$$

We use Lemmas 3.2 and 3.3 to find the general form of $\mathcal{J}_{\rho}\left(\mathfrak{K}_{m}^{k, p}, \mathfrak{S}_{m^{\prime}}^{k^{\prime}, p}\right)$ for any $\rho>0$. We obtain

$$
\begin{aligned}
\mathcal{J}_{\rho}\left(\mathfrak{K}_{m}^{k, p}, \mathfrak{S}_{m^{\prime}}^{k^{\prime}, p}\right) & =\sum_{j=0}^{m} \sum_{j^{\prime}=0}^{m^{\prime}} \sum_{q=0}^{j+1} \sum_{q^{\prime}=0}^{j^{\prime}} \mathcal{J}_{j, q, j^{\prime}, q^{\prime}}^{k, p ;}\left(i \zeta^{2}\right)^{j+j^{\prime}} \rho^{-k+k^{\prime}+2 j+2 j^{\prime}} \log ^{q+q^{\prime}} \rho \\
& =\sum_{\underline{j}=0}^{m+m^{\prime}} \sum_{\underline{q}=0}^{\underline{j}+1} \underline{\mathcal{J}}_{\underline{j}, \underline{q}}^{k, p}\left(k^{\prime}\left(i \zeta^{2}\right)^{j} \rho^{-k+k^{\prime}+2 \underline{j}} \log \underline{q} \rho .\right.
\end{aligned}
$$

Fix $R>0$ and let $\varepsilon$ go to 0 . The expression (38) yields that $\mathcal{J}_{R}\left(\mathfrak{K}_{m}^{k, p}, \mathfrak{S}_{m^{\prime}}^{k^{\prime}, p}\right)-\mathcal{J}_{\varepsilon}\left(\mathfrak{K}_{m}^{k, p}, \mathfrak{S}_{m^{\prime}}^{k^{\prime}, p}\right)$ is bounded independently of $\varepsilon<R$, which means in particular that $\mathcal{J}_{\varepsilon}\left(\mathfrak{K}_{m}^{k, p}, \mathfrak{S}_{m^{\prime}}^{k^{\prime}, p}\right)$ is bounded independently of $\varepsilon<R$. Considering (40) for $\rho=\varepsilon$, we deduce that the coefficients $\underline{\mathcal{J}}_{\underline{j}, \underline{q}}^{k, p ; k^{\prime}}$ attached to unbounded

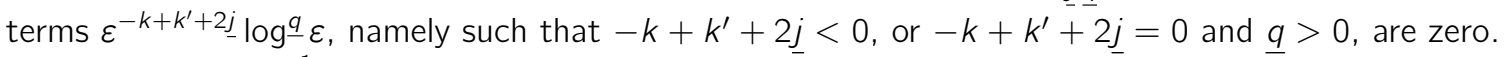
Hence, setting $\nu=\frac{1}{2}\left(k-k^{\prime}\right)$, we find

$$
\mathcal{J}_{\rho}\left(\mathfrak{K}_{m}^{k, p}, \mathfrak{S}_{m^{\prime}}^{k^{\prime}, p}\right)= \begin{cases}\left(i \zeta^{2}\right)^{\nu} \underline{\mathcal{J}}_{\nu, 0}^{k, p ; k^{\prime}}+\sum_{\underline{j}=\nu+1}^{m+m^{\prime}} \sum_{\underline{q}=0}^{\underline{j}+1} \underline{\mathcal{J}}_{\underline{j}, \underline{q}}^{k, p ; k^{\prime}}\left(i \zeta^{2}\right)^{\underline{j}} \rho^{-k+k^{\prime}+2 \underline{j} \log \underline{q} \rho} & \text { if } \nu \in \mathbb{N}, \\ \sum_{\underline{j}=[\nu]+1}^{m+m^{\prime}} \sum_{\underline{q}=0}^{\underline{j}+1} \underline{\mathcal{J}}_{\underline{j}, \underline{q}}^{k, p ; k^{\prime}}\left(i \zeta^{2}\right)^{j}-\rho^{-k+k^{\prime}+2 \underline{j}} \underline{\log \underline{q} \rho} & \text { if } \nu \notin \mathbb{N} .\end{cases}
$$

We note that $\lim _{\varepsilon \rightarrow 0} \mathcal{J}_{\varepsilon}\left(\mathfrak{K}_{m}^{k, p}, \mathfrak{S}_{m^{\prime}}^{k^{\prime}, p}\right)$ exists and is $\left(i \zeta^{2}\right)^{\nu} \underline{\mathcal{J}}_{\nu, 0}^{k, p ; k^{\prime}}$ if $\nu \in \mathbb{N}$ and 0 if not. Letting $\varepsilon \rightarrow 0$ in (37)-(38) we find in all cases

$$
\sum_{\underline{j}=[\nu]+1}^{m+m^{\prime}} \sum_{\underline{q}=0}^{\underline{j}+1} \underline{\mathcal{J}}_{\underline{j}, \underline{q}}^{k, p ; k^{\prime}}\left(i \zeta^{2}\right)^{j}-R^{-k+k^{\prime}+2 \underline{j}} \log \underline{q} R \underset{R \rightarrow 0}{=} \zeta^{2 m+2} \mathcal{O}\left(R^{-k+k^{\prime}+2 m+2} \log ^{m+2} R\right) .
$$

Therefore the coefficients $\underline{\mathcal{J}}_{\underline{j}, \underline{q}}^{k, p ; k^{\prime}}$ are zero if $-k+k^{\prime}+2 \underline{j}<-k+k^{\prime}+2 m+2$, i.e. if $\underline{j}<m+1$. Finally

$$
\mathcal{J}_{\rho}\left(\mathfrak{K}_{m}^{k, p}, \mathfrak{S}_{m^{\prime}}^{k^{\prime}, p}\right)= \begin{cases}\left(i \zeta^{2}\right)^{\nu} \underline{\mathcal{J}}_{\nu, 0}^{k, p ; k^{\prime}}+\sum_{\underline{j}=m+1}^{m+m^{\prime}} \sum_{\underline{q}=0}^{\underline{j}+1} \underline{\mathcal{J}}_{\underline{j}, \underline{q}}^{k, p ; k^{\prime}}\left(i \zeta^{2}\right)^{\underline{j}} \rho^{-k+k^{\prime}+2 \underline{j}} \log \underline{q} \rho & \text { if } \nu \in \mathbb{N}, \\ \sum_{j=m+1}^{m+m^{\prime}} \sum_{\underline{q}=0}^{\underline{j}+1} \underline{\mathcal{J}}_{\underline{j}, \underline{q}}^{k, p ; k^{\prime}}\left(i \zeta^{2}\right)^{j} \rho^{-k+k^{\prime}+2 \underline{j}} \log \underline{q} \rho & \text { if } \nu \notin \mathbb{N} .\end{cases}
$$


Taking the largest term in the remainders of equality (42), we find

$$
\mathcal{J}_{R}\left(\mathfrak{K}_{m}^{k, p}, \mathfrak{S}_{m^{\prime}}^{k^{\prime}, p}\right) \underset{R \rightarrow 0}{=} \begin{cases}\left(i \zeta^{2}\right)^{\nu} \underline{\mathcal{J}}_{\nu, 0}^{k, p ; k^{\prime}}+\mathcal{O}\left(\left(i \zeta^{2}\right)^{m+1} R^{-k+k^{\prime}+2 m+2} \log ^{m+2} R\right) & \text { if } \nu \in \mathbb{N} \\ \mathcal{O}\left(\left(i \zeta^{2}\right)^{m+1} R^{-k+k^{\prime}+2 m+2} \log ^{m+2} R\right) & \text { if } \nu \notin \mathbb{N}\end{cases}
$$

Recall that $\nu=\frac{1}{2}\left(k-k^{\prime}\right)$. The case $\nu \in \mathbb{N}$ amounts to considering $k^{\prime}=k-2 \ell$ with $\ell \leqslant[k / 2]$. Setting

$$
\mathcal{J}^{k, p ; k-2 \ell, p}=\left(i \zeta^{2}\right)^{\ell} \underline{\mathcal{J}}_{\ell, 0}^{k, p ; k-2 \ell},
$$

and $\mathcal{J}^{k, p ; k^{\prime}, p^{\prime}}=0$ otherwise, we have proved the theorem.

Proposition 3.7. Let $k \in \mathbb{N} \backslash\{0,1\}$ and $p \in\{0,1\}$. For any $\ell \in 1, \ldots,[k / 2]$, the coefficients $\mathcal{J}^{k, p ; k-2 \ell, p}$ introduced in (35) are given by

$$
\mathcal{J}^{k, p ; k-2 \ell, p}=\left(i \zeta^{2}\right)^{\ell} \sum_{j=0}^{\ell} \int_{0}^{2 \pi} \Psi_{j, 0}^{k, p}(\theta)\left[2(k-2 j) \Phi_{\ell-j, 0}^{k-2 \ell, p}(\theta)+\Phi_{\ell-j, 1}^{k-2 \ell, p}(\theta)\right]-\Psi_{j, 1}^{k, p}(\theta) \Phi_{\ell-j, 0}^{k-2 \ell, p}(\theta) d \theta,
$$

with the convention that $\Phi_{0,1}^{k-2 \ell, p}=0$ and $\Psi_{0,1}^{k, p}=0$.

Proof. Recall that $\mathcal{J}^{k, p ; k-2 \ell, p}=\left(i \zeta^{2}\right)^{\ell} \underline{\mathcal{J}}_{\ell, 0}^{k, p ; k-2 \ell}$ where from (43)

$$
\mathcal{J}_{R}\left(\mathfrak{K}_{m}^{k, p}, \mathfrak{S}_{m^{\prime}}^{k-2 \ell, p}\right) \underset{R \rightarrow 0}{=}\left(i \zeta^{2}\right)^{\ell} \underline{\mathcal{J}}_{\ell, 0}^{k, p ; k-2 \ell}+\mathcal{O}\left(\left(i \zeta^{2}\right)^{m+1} R^{-2 \ell+2 m+2} \log ^{m+2} R\right),
$$

with $m^{\prime} \geqslant m \geqslant 1$; moreover

$$
\mathcal{J}_{R}\left(\mathfrak{K}_{m}^{k, p}, \mathfrak{S}_{m^{\prime}}^{k-2 \ell, p}\right)=\int_{r=R}\left(\mathfrak{K}_{m}^{k, p} \partial_{r} \mathfrak{S}_{m^{\prime}}^{k-2 \ell, p}-\mathfrak{S}_{m^{\prime}}^{k-2 \ell, p} \partial_{r} \mathfrak{K}_{m}^{k, p}\right) R \mathrm{~d} \theta .
$$

Comparing (39) and (40), it is straightforward that the useful terms for computing $\mathcal{J}^{k, p ; k-2 \ell, p}$ are the terms whose powers in $r$ and in $\log r$ equal 0 in the product $r \mathfrak{K}_{m}^{k, p} \partial_{r} \mathfrak{S}_{m^{\prime}}^{k-2 \ell, p}$ and in the product $r \mathfrak{S}_{m^{\prime}}^{k-2 \ell, p} \partial_{r} \mathfrak{K}_{m}^{k, p}$.

From (22) and (25), straightforward calculi lead to

$$
\begin{aligned}
& \partial_{r} \mathfrak{S}^{k, p}(r, \theta)=r^{k-1} \sum_{j \geqslant 0}\left(i \zeta^{2}\right)^{j} r^{2 j} \sum_{n=0}^{j} \log ^{n} r\left((k+2 j) \Phi_{j, n}^{k, p}(\theta)+(n+1) \Phi_{j, n+1}^{k, p}(\theta)\right), \\
& \partial_{r} \mathfrak{K}^{k, p}(r, \theta)=r^{-k-1} \sum_{j \geqslant 0}\left(i \zeta^{2}\right)^{j} r^{2 j} \sum_{n=0}^{j+1} \log ^{n} r\left((-k+2 j) \Psi_{j, n}^{k, p}(\theta)+(n+1) \Psi_{j, n+1}^{k, p}(\theta)\right),
\end{aligned}
$$

with the convention that $\Phi_{j, n}^{k, p}=0, \forall n>j$ and $\Psi_{j, n}^{k, p}=0$, if $(n>(j+1))$ or $((n>j)$ and $k$ odd $)$ or $((n>j)$ and $k$ even and $(j<k / 2))$. From (25) and (45), the terms of the product $r \mathfrak{K}_{m}^{k, p} \partial_{r} \mathfrak{S}_{m^{\prime}}^{k-2 \ell, p}$, whose powers in $r$ and $\log r$ equal 0 are given by

$$
\left(i \zeta^{2}\right)^{\ell} \sum_{j=0}^{l} \int_{0}^{2 \pi} \Psi_{j, 0}^{k, p}(\theta)\left[(k-2 j) \Phi_{\ell-j, 0}^{k-2 \ell, p}(\theta)+\Phi_{\ell-j, 1}^{k-2 \ell, p}(\theta)\right] d \theta,
$$

and similarly, from (22) and (46), the terms of the product $r \mathfrak{S}_{m^{\prime}}^{k-2 \ell, p} \partial_{r} \mathfrak{K}_{m}^{k, p}$, whose powers in $r$ and $\log r$ equal 0 are

$$
\left(i \zeta^{2}\right)^{\ell} \sum_{j=0}^{I} \int_{0}^{2 \pi} \Phi_{I-j, 0}^{k-2 \ell, p}(\theta)\left[(-k+2 j) \Psi_{j, 0}^{k, p}(\theta)+\Psi_{j, 1}^{k, p}(\theta)\right] d \theta
$$

Making the difference of both contributions, we obtain (44).

RR n ${ }^{\circ} 8204$ 


\section{Leading singularities and their first shadows in complex vari- ables}

According to the previous section, the explicit calculation of both primal and dual singularities is essential to obtain a precise description of the magnetic potential $\mathcal{A}$ for numerical purposes. The heuristics of such a calculus have been described in subsections 3.1 and 3.2. It consists in deriving the shadow at any order of the leading singularities (primal or dual depending on which kind of singularities is being considered). The difficulty of these calculations lies in the transmission conditions (continuity of the potential $\mathcal{A}$ and of its normal derivative) across the boundary $\mathcal{G}$, that have to be imposed on the angular functions $\Phi$ of the space $S^{\lambda}$. The use of appropriate complex variables like in [2] instead of the polar coordinates $(r, \theta)$ simplifies drastically the calculations. Indeed, in our case, it will be sufficient to use an Ansatz using two complex variables $z_{+}$and $z_{-}$(in $\mathcal{S}_{-}$and $\mathcal{S}_{+}$) involving only integer powers of $z_{ \pm}$, $\bar{z}_{ \pm}, \log z_{ \pm}$, and $\log \bar{z}_{ \pm}$. Thus, it is much less complex than a general Ansatz using polar coordinates without further information. The aim of this section is to derive the first shadow terms of both primal and dual leading singularities and to present a systematic method of calculus if more terms are needed.

\subsection{Formalism in complex variables}

Let us present the appropriate formalism in complex variables. Setting $z=r e^{i \theta}=x+i y$ it is obvious that

$$
\partial_{z}=\frac{1}{2}\left(\partial_{x}-i \partial_{y}\right) \quad \text { and } \quad \partial_{\bar{z}}=\frac{1}{2}\left(\partial_{x}+i \partial_{y}\right)
$$

from which we deduce

$$
r \partial_{r}=z \partial_{z}+\bar{z} \partial_{\bar{z}}, \quad \partial_{\theta}=\mathrm{i}\left(z \partial_{z}-\bar{z} \partial_{\bar{z}}\right) \quad \text { and } \quad \Delta=4 \partial_{z} \partial_{\bar{z}}
$$

The leading terms $\mathfrak{s}_{0}^{k, p}=\mathfrak{s}^{k, p}$ and $\mathfrak{k}_{0}^{k, p}=\mathfrak{k}^{k, p}$ of the primal and dual singularities have a natural expression in complex variable: Setting for any nonnegative integer $k:^{3}$

$$
\mathrm{s}_{0}^{k}\left(r e^{\mathrm{i} \theta}\right)=\mathfrak{s}_{0}^{k, 0}(r, \theta)+\mathfrak{i} \mathfrak{s}_{0}^{k, 1}(r, \theta) \quad \text { and } \quad \mathrm{k}_{0}^{k}\left(r e^{\mathrm{i} \theta}\right)=\mathfrak{k}_{0}^{k, 0}(r, \theta)-i \mathfrak{k}_{0}^{k, 1}(r, \theta),
$$

we obtain

$$
\forall k \in \mathbb{N} \quad s_{0}^{k}(z)=z^{k} \quad \text { and }\left\{\begin{array}{l}
k_{0}^{k}(z)=\frac{1}{2 k \pi} z^{-k} \quad \text { if } \quad k \in \mathbb{N} \backslash\{0\}, \\
k_{0}^{0}(z)=-\frac{1}{2 \pi} \log z \quad \text { if } \quad k=0 .
\end{array}\right.
$$

The idea is to take advantage of formulas (47) and (48) to solve problems (19)-(20) and their analogues for dual functions, in order to determine shadow terms.

Complex variables in $\mathcal{S}_{-}$and $\mathcal{S}_{+}$. Intuitively, since the shadows $\mathfrak{s}_{j}^{k, p}$ and $\mathfrak{k}_{j}^{k, p}$ belong respectively to $\mathrm{S}^{k+2 j}$ and $\mathrm{S}^{-k+2 j}$, the corresponding function of the complex variable $\mathrm{s}_{j}^{k}$ and $\mathrm{k}_{j}^{k}$ should involve terms in $\log ^{q} z, q \in \mathbb{N}$. Note however that despite $\log (z)$ is well-defined in $\mathcal{S}_{-}$with its branch cut on $\mathbb{R}^{-}$, it is not defined in $\mathcal{S}_{+}$(which contains $\mathbb{R}^{-}$).

To avoid such a problem we introduce two complex variables to perform the calculations with the same determination of the complex logarithm. Since $\mathcal{G}$ is the broken line $\{z:|\arg (z)|=\omega / 2\}$ we define $z_{-}$and $z_{+}$as

$$
z_{-}=z, \quad z_{+}=-z,
$$

\footnotetext{
${ }^{3}$ For uniformity of presentation we complete the sets of functions $\mathfrak{s}_{0}^{k, p}$ and $\mathfrak{k}_{0}^{k, p}$ by the convention that $\mathfrak{s}_{0}^{0,1}=0$ and $\mathfrak{k}_{0}^{0,1}=\theta$.
} 
which ensures that $\log \left(z_{-}\right)$and $\log \left(z_{+}\right)$are well defined respectively for $z_{-} \in \mathcal{S}_{-}$and $z_{+} \in \mathcal{S}_{+}$, where $\log$ is the usual complex logarithm with its branch cut on $\mathbb{R}^{-}$. For the sake of simplicity, we simply denote $z_{-}$by $z$, and in $(r, \theta)$-coordinates we have

$$
z=r e^{i \theta}, \quad z_{+}=r e^{i \theta_{+}}, \quad \text { where } \quad \theta_{+}= \begin{cases}\theta-\pi & \text { if } \theta \in(0, \pi], \\ \theta+\pi & \text { if } \theta \in[-\pi, 0) .\end{cases}
$$

As previously presented, the two primal leading singularities, $\mathfrak{s}_{0}^{k, 0}$ and $\mathfrak{s}_{0}^{k, 1}$, are the respective real and imaginary parts of $s_{0}^{k}(z)=z^{k}$, for $k \in \mathbb{N}$, which writes $s_{0}^{k}=\left(\chi_{0}^{k}, \xi_{0}^{k}\right)$ in the variables $\left(z, z_{+}\right)$where

$$
\begin{cases}\chi_{0}^{k}(z)=z^{k}, & |\arg z| \leqslant \frac{\omega}{2}, \\ \xi_{0}^{k}\left(z_{+}\right)=(-1)^{k}\left(z_{+}\right)^{k}, & \left|\arg z_{+}\right| \leqslant \pi-\frac{\omega}{2} .\end{cases}
$$

Shadows in complex variables For any $k \in \mathbb{N}$ and $j \geqslant 1$, we define the couples $s_{j}^{k}:=\left(\chi_{j}^{k}(z), \xi_{j}^{k}\left(z_{+}\right)\right)$ and $\mathrm{k}_{j}^{k}:=\left(\zeta_{j}^{k}(z), \eta_{j}^{k}\left(z_{+}\right)\right)$by

$$
\left\{\begin{array}{c}
\chi_{j}^{k}(z)=\mathfrak{s}_{j}^{k, 0}(r, \theta)+\mathrm{i} \mathfrak{s}_{j}^{k, 1}(r, \theta), \quad \text { and } \quad \zeta_{j}^{k}(z)=\mathfrak{k}_{j}^{k, 0}(r, \theta)-\mathrm{i} \mathfrak{k}_{j}^{k, 1}(r, \theta), \quad \text { if }|\theta| \leqslant \frac{\omega}{2}, \\
\xi_{j}^{k}\left(z_{+}\right)=\mathfrak{s}_{j}^{k, 0}(r, \theta)+\mathrm{i} \mathfrak{s}_{j}^{k, 1}(r, \theta), \quad \text { and } \quad \zeta_{j}^{k}\left(z_{+}\right)=\mathfrak{k}_{j}^{k, 0}(r, \theta)-\mathrm{i} \mathfrak{k}_{j}^{k, 1}(r, \theta), \quad \text { if }|\theta| \geqslant \frac{\omega}{2} .
\end{array}\right.
$$

The interior problem (19) translates into $\partial_{z} \partial_{\bar{z}} \chi_{j}^{k}=\chi_{j-1}^{k}$ in $\mathcal{S}_{-}$and $\partial_{z_{+}} \partial_{\bar{z}_{+}} \xi_{j}^{k}=0$ in $\mathcal{S}_{+}$, and similarly for (24). The transmission conditions (20) write equivalently in polar coordinates $\left[r \partial_{r} \mathfrak{s}_{j}^{k, p}\right]_{\mathcal{G}}=0$ and $\left[\partial_{\theta} \mathfrak{s}_{j}^{k, p}\right]_{\mathcal{G}}=0$ and similarly for $\mathfrak{k}_{j}^{k, p}$. As far as primal singularities are concerned, the degree of (quasi) homogeneity of $\mathfrak{s}_{j}^{k, p}$ is never 0 and the transmission conditions are equivalent to $\left[\partial_{z} s_{j}^{k}\right]_{\mathcal{G}}=0$ and $\left[\partial_{\bar{z}} \mathrm{~s}_{j}^{k}\right]_{\mathcal{G}}=0$. The computation of the primal shadow terms of order $j \geqslant 1$ consists now in finding $\left(\chi_{j}^{k}(z), \xi_{j}^{k}\left(z_{+}\right)\right)$such that

$$
\begin{aligned}
\partial_{z} \partial_{\bar{z}} \chi_{j}^{k}=\chi_{j-1}^{k} & \text { in } \mathcal{S}_{-}, \\
\partial_{z_{+}} \partial_{\bar{z}_{+}} \xi_{j}^{k}=0, & \text { in } \mathcal{S}_{+}, \\
\partial_{z} \chi_{j}^{k}+\partial_{z_{+}} \xi_{j}^{k}=0 & \text { on } \mathcal{G}, \\
\partial_{\bar{z}} \chi_{j}^{k}+\partial_{\bar{z}_{+}} \xi_{j}^{k}=0 & \text { on } \mathcal{G} .
\end{aligned}
$$

If $-k+2 j$ is not equal to 0 , the dual shadow terms of order $j \geqslant 1$ are determined by solutions $\left(\zeta_{j}^{k}(z), \eta_{j}^{k}\left(z_{+}\right)\right)$to problem

$$
\begin{aligned}
\partial_{z} \partial_{\bar{z}} \zeta_{j}^{k}=\zeta_{j-1}^{k} & \text { in } \mathcal{S}_{-}, \\
\partial_{z_{+}} \partial_{\bar{z}_{+}} \eta_{j}^{k}=0, & \text { in } \mathcal{S}_{+}, \\
\partial_{z} \zeta_{j}^{k}+\partial_{z_{+}} \eta_{j}^{k}=0 & \text { on } \mathcal{G}, \\
\partial_{\bar{z}} \zeta_{j}^{k}+\partial_{\bar{z}_{+}} \eta_{j}^{k}=0 & \text { on } \mathcal{G} .
\end{aligned}
$$

Finally if $-k+2 j$ vanishes, $\left(\zeta_{j}^{k}(z), \eta_{j}^{k}\left(z_{+}\right)\right)$satisfies

$$
\begin{aligned}
\partial_{z} \partial_{\bar{z}} \zeta_{j}^{k}=\zeta_{j-1}^{k} & \text { in } \mathcal{S}_{-}, \\
\partial_{z_{+}} \partial_{\bar{z}_{+}} \eta_{j}^{k}=0, & \text { in } \mathcal{S}_{+}, \\
z \partial_{z} \zeta_{j}^{k}-\bar{z} \partial_{\bar{z}} \zeta_{j}^{k}-z_{+} \partial_{z_{+}} \eta_{j}^{k}+\bar{z}_{+} \partial_{\bar{z}_{+}} \eta_{j}^{k}=0 & \text { on } \mathcal{G}, \\
\zeta_{j}^{k}-\eta_{j}^{k}=0 & \text { on } \mathcal{G} .
\end{aligned}
$$

RR n ${ }^{\circ} 8204$ 
Performing the calculations, we will find that the following Ansatz spaces are the correct ones to solve the above three problems: For $\lambda \in \mathbb{Z}$ and $(\ell, n) \in \mathbb{N}^{2}$ we define the $\mathbb{R}$-vector spaces $\mathscr{Z}_{\ell, n}^{\lambda, \pm}, \mathscr{Z}_{\ell, n}^{\lambda}$ and $\mathbb{S}_{\ell, n}^{\lambda}$ by

$$
\begin{aligned}
\mathscr{Z}_{\ell, n}^{\lambda, \pm} & =\operatorname{Span}_{\mathbb{R}}\left\{z_{ \pm}^{\lambda-\mu} \bar{z}_{ \pm}^{\mu} \log ^{q}\left(z_{ \pm}\right), \text {with } 0 \leqslant \mu \leqslant \ell, 0 \leqslant q \leqslant n\right\} \\
\mathbb{S}_{\ell, \pm}^{\lambda, \pm} & =\mathscr{Z}_{\ell, n}^{\lambda, \pm}+\overline{\mathscr{Z}}_{\ell, n}^{\lambda, \pm}, \\
\mathbb{S}_{\ell, n}^{\lambda} & =\left\{u=\left(v\left(z_{-}\right), w\left(z_{+}\right)\right), \text {with } \quad v \in \mathbb{S}_{\ell, n}^{\lambda,-} \text { and } w \in \mathbb{S}_{\ell, n}^{\lambda,+}\right\},
\end{aligned}
$$

where $\overline{\mathscr{Z}_{\ell, n}^{\lambda, \pm}}$ is the space of conjugates $\bar{u}$ with $u \in \mathscr{Z}_{\ell, n}^{\lambda, \pm}$. Note that $\mathbb{S}_{\ell, n}^{\lambda}$ is a finite dimensional subspace of the space $\mathrm{T}^{\lambda}$ introduced in (17).

Remark 4.1. We emphasize that the spaces $\mathscr{Z}_{\ell, n}^{\lambda, \pm}$ are $\mathbb{R}$-vector spaces, and not $\mathbb{C}$-vector spaces, which will be important in the foreseen calculus.

We are going to prove the following theorem in the next subsections (for $j=1$ ) and in Appendix A (for $j \geqslant 2$ ).

Theorem 4.2. Let $k \in \mathbb{N}$ and $j \geqslant 1$. Then the primal shadow $s_{j}^{k}$ in complex variables belongs to $\mathbb{S}_{j, j}^{k+2 j}$. If $-k+2 j<0$, the dual shadow $k_{j}^{k}$ belongs to $\mathbb{S}_{j, j}^{-k+2 j}$ and if $-k+2 j \geqslant 0, k_{j}^{k}$ belongs to $\mathbb{S}_{j, j+1}^{-k+2 j}$. The functions $\mathfrak{s}_{j}^{k, p}$ and $\mathrm{s}_{j}^{k}$ (respectively $\mathfrak{k}_{j}^{k, p}$ and $\mathrm{k}_{j}^{k}$ ) are linked by

$$
\left\{\begin{array}{lll}
\mathfrak{s}_{j}^{k, 0}(r, \theta)=\operatorname{Re}\left(\mathrm{s}_{j}^{k}\left(r e^{\mathrm{i} \theta}\right)\right), & \mathfrak{k}_{j}^{k, 0}(r, \theta)=\operatorname{Re}\left(\mathrm{k}_{j}^{k}\left(r e^{\mathrm{i} \theta}\right)\right), & \text { if } k \in \mathbb{N} \\
\mathfrak{s}_{j}^{k, 1}(r, \theta)=\operatorname{Im}\left(\mathrm{s}_{j}^{k}\left(r e^{\mathrm{i} \theta}\right)\right), & \mathfrak{k}_{j}^{k, 1}(r, \theta)=-\operatorname{Im}\left(\mathrm{k}_{j}^{k}\left(r e^{\mathrm{i} \theta}\right)\right) & \text { if } k \in \mathbb{N} \backslash\{0\} .
\end{array}\right.
$$

\subsection{Calculation of the shadow function generated by $z^{k}$ and $\log z$}

In this section, we derive the shadow term generated by $z^{k}$ in the space $\mathbb{S}_{1,1}^{k+2}$ for $k \in \mathbb{Z} \backslash\{-2\}$ and in $\mathbb{S}_{1,2}^{0}$ if $k=-2$. We will also find the shadow term generated by $\log z$ in $\mathbb{S}_{1,2}^{2}$. The shadows of these functions are important since the leading term of the primal functions are the function $z^{k}$ with $k \in \mathbb{N}$, while the leading term of the dual functions are $\log z$ and the functions $z^{-k}$ with $k \in \mathbb{N} \backslash\{0\}$.

Proposition 4.3. Let $k \in \mathbb{Z} \backslash\{-2,-1\}$. A particular solution $u^{k}=\left(v^{k}, w^{k}\right)$ to the following problem

$$
\begin{aligned}
\partial_{z} \partial_{\bar{z}} v^{k}=z^{k} & \text { in } \mathcal{S}_{-}, \\
\partial_{z_{+}} \partial_{\bar{z}_{+}} w^{k}=0, & \text { in } \mathcal{S}_{+}, \\
\partial_{z} v^{k}+\partial_{z_{+}} w^{k}=0 & \text { on } \mathcal{G}, \\
\partial_{\bar{z}} v^{k}+\partial_{\bar{z}_{+}} w^{k}=0 & \text { on } \mathcal{G},
\end{aligned}
$$

writes

$$
\begin{aligned}
v^{k}(z) & =\frac{\sin \omega}{\pi(k+2)} z^{k+2} \log z+\frac{\sin (k+1) \omega}{\pi(k+1)(k+2)} \bar{z}^{k+2} \log \bar{z}-\frac{\cos \omega}{k+2} z^{k+2}+\frac{z^{k+1} \bar{z}}{k+1} \\
(-1)^{k} w^{k}\left(z_{+}\right) & =\frac{\sin \omega}{\pi(k+2)} z_{+}^{k+2} \log z_{+}+\frac{\sin (k+1) \omega}{\pi(k+1)(k+2)} \bar{z}_{+}^{k+2} \log \bar{z}_{+}+\frac{\cos (k+1) \omega}{(k+1)(k+2)} \bar{z}_{+}^{k+2} .
\end{aligned}
$$


Proof. Since the function $z^{k+1} \bar{z} /(k+1)$ satisfies

$$
\partial_{z} \partial_{\bar{z}}\left(z^{k+1} \bar{z} /(k+1)\right)=z^{k}
$$

we suppose that the solution $(v, w)=\left(v^{k}, w^{k}\right)$ to (56) writes

$$
\begin{cases}v(z) & =a z^{k+2} \log z+a^{\prime} \bar{z}^{k+2} \log \bar{z}+b z^{k+2}+z^{k+1} \bar{z} /(k+1), \\ (-1)^{k} w\left(z_{+}\right) & =a z_{+}^{k+2} \log z_{+}+a^{\prime} \bar{z}_{+}^{k+2} \log \bar{z}_{+}+b^{\prime} \bar{z}_{+}^{k+2} .\end{cases}
$$

Observe that equations (56a) and (56b) are satisfied by the Ansatz, for any complex numbers $a$, $b, a^{\prime}$ and $b^{\prime}$. Jump conditions will determine $a, b, a^{\prime}$ and $b^{\prime}$ in order (56) to be satisfied.

Equations (56c)-(56d) lead to four conditions for the coefficients $a, b, a^{\prime}$ and $b^{\prime}$, since both equalities hold in $\arg (z)=\omega / 2$ (i.e. $\left.\arg \left(z_{+}\right)=\omega / 2-\pi\right)$ and $\arg (z)=-\omega / 2$ (i.e. $\arg \left(z_{+}\right)=\pi-\omega / 2$ ). For instance write equation (56c) in $\arg (z)=\omega / 2$. Applying the following equalities

$$
\begin{array}{ll}
\forall z \in \mathcal{S}_{-}, & \partial_{z} v(z)=a\left((k+2) z^{k+1} \log z+z^{k+1}\right)+b(k+2) z^{k+1}+z^{k} \bar{z}, \\
\forall z_{+} \in \mathcal{S}_{+}, & \partial_{z_{+}} w\left(z_{+}\right)=(-1)^{k} a\left((k+2) z_{+}^{k+1} \log z_{+}+z_{+}^{k+1}\right),
\end{array}
$$

respectively in $z=r e^{i \omega / 2}$ and $z_{+}=r e^{i(\omega / 2-\pi)}$ and using constraint (56c) imply

$$
\partial_{z} v\left|\arg (z)=\omega / 2+\partial_{z_{+}} w\right|_{\arg \left(z_{+}\right)=\omega / 2-\pi}=r^{k+1} e^{i(k+1) \omega / 2}\left(i \pi a(k+2)+b(k+2)+e^{-i \omega}\right),
$$

and similarly in $z=r e^{-i \omega / 2}$ and $z_{+}=r e^{i(-\omega / 2+\pi)}$ :

$$
\left.\partial_{z} v\right|_{\arg (z)=-\omega / 2}+\left.\partial_{z_{+}} w\right|_{\arg \left(z_{+}\right)=-\omega / 2+\pi}=r^{k+1} e^{-i(k+1) \omega / 2}\left(-i \pi a(k+2)+b(k+2)+e^{i \omega}\right) .
$$

Therefore $\partial_{z} v+\partial_{z_{+}} w$ vanishes on $\mathcal{G}$ iff

$$
\begin{cases}\mathrm{i} \pi a+b & =-\mathrm{e}^{-\mathrm{i} \omega} /(k+2), \\ -\mathrm{i} \pi a+b & =-\mathrm{e}^{\mathrm{i} \omega} /(k+2),\end{cases}
$$

hence

$$
a=\frac{\sin \omega}{\pi(k+2)} \text { and } b=-\frac{\cos \omega}{k+2} .
$$

Very similar computations imply that the jump $\partial_{\bar{z}} v+\partial_{\bar{z}_{+}} w$ vanishes in $\theta=\omega / 2$ iff

$$
-(k+2) \mathrm{e}^{-\mathrm{i}(k+1) \omega / 2} \mathrm{i} \pi a^{\prime}-(k+2) \mathrm{e}^{-\mathrm{i}(k+1) \omega / 2} b^{\prime}+\mathrm{e}^{\mathrm{i}(k+1) \omega / 2} /(k+1)=0 .
$$

For $\theta=-\omega / 2$, we have

$$
(k+2) \mathrm{e}^{\mathrm{i}(k+1) \omega / 2} \mathrm{i} \pi a^{\prime}-(k+2) \mathrm{e}^{\mathrm{i}(k+1) \omega / 2} b^{\prime}+\mathrm{e}^{-\mathrm{i}(k+1) \omega / 2} /(k+1)=0 .
$$

Hence, $\partial_{\bar{z}} v+\partial_{\bar{z}_{+}} w$ vanishes on $\mathcal{G}$ iff

$$
\begin{cases}\mathrm{i} \pi a^{\prime}+b^{\prime} & =\mathrm{e}^{\mathrm{i}(k+1) \omega} /(k+1)(k+2) \\ -\mathrm{i} \pi a^{\prime}+b^{\prime} & =\mathrm{e}^{-\mathrm{i}(k+1) \omega} /(k+1)(k+2)\end{cases}
$$

from which we infer

$$
a^{\prime}=\frac{\sin (k+1) \omega}{\pi(k+1)(k+2)} \quad \text { and } \quad b^{\prime}=\frac{\cos (k+1) \omega}{(k+1)(k+2)} .
$$

Therefore, $u^{k}=\left(v^{k}, w^{k}\right)$ writes (57).

RR n ${ }^{\circ} 8204$ 
If $k=-1$, a particular solution to (56) necessarily involves the function $\bar{z} \log z$ since $\partial_{z} \partial_{\bar{z}}(\bar{z} \log z)=$ $z^{-1}$. We therefore have

Proposition 4.4. For $k=-1$, a particular solution $u^{-1}=\left(v^{-1}, w^{-1}\right)$ to (56) writes

$$
\begin{aligned}
v^{-1}(z) & =\frac{\sin \omega}{\pi} z \log z+\frac{\omega-\pi}{\pi} \bar{z} \log \bar{z}-\cos \omega z+\bar{z} \log z \\
w^{-1}\left(z_{+}\right) & =-\frac{\sin \omega}{\pi} z_{+} \log z_{+}-\frac{\omega}{\pi} \bar{z}_{+} \log \bar{z}_{+}+\bar{z}_{+} .
\end{aligned}
$$

Proof. Similarly to (58), for $k=-1$ we suppose that the solution $(v, w)=\left(v^{k}, w^{k}\right)$ to (56) writes

$$
\begin{cases}v(z) & =a z \log z+a^{\prime} \bar{z} \log \bar{z}+b z+\bar{z} \log z \\ w\left(z_{+}\right) & =-a z_{+} \log z_{+}-\left(a^{\prime}+1\right) \bar{z}_{+} \log \bar{z}_{+}-b^{\prime} \bar{z}_{+}\end{cases}
$$

Very similar computations using the jump conditions (56c) on $\mathcal{G}$ imply necessarily

$$
\left\{\begin{array}{l}
\mathrm{i} a \pi+b=-e^{-\mathrm{i} \omega} \\
\mathrm{i} a \pi-b=e^{\mathrm{i} \omega}
\end{array}\right.
$$

and using (56d), this leads to

$$
\left\{\begin{aligned}
\mathrm{i} a^{\prime} \pi+b^{\prime}+1 & =\mathrm{i}(\omega-\pi) \\
\mathrm{i} a^{\prime} \pi-b^{\prime}-1 & =\mathrm{i}(\omega-\pi)
\end{aligned}\right.
$$

hence the proposition.

The case $k=-2$ is quite different since the source term belongs to $\mathrm{T}^{-2}$ of Lemma 3.1, hence the degree of the shadow (as polynomial in $\log (r)$ ) is bounded by 2 instead of 1 . The following proposition shows that it is actually equal to 2 .

Proposition 4.5. For $k=-2$, a particular solution $u^{-2}=\left(v^{-2}, w^{-2}\right)$ to (54) writes

$$
\begin{aligned}
v^{-2}(z) & =\frac{1}{2 \pi} \sin \omega \log ^{2} z+\frac{1}{2 \pi} \sin \omega \log ^{2} \bar{z}-\frac{1}{\pi}(\sin \omega+(2 \pi-\omega) \cos \omega) \log z-z^{-1} \bar{z} \\
w^{-2}\left(z_{+}\right) & =\frac{1}{2 \pi} \sin \omega \log ^{2} z_{+}+\frac{1}{2 \pi} \sin \omega \log ^{2} \bar{z}_{+}-\frac{1}{\pi}(\sin \omega+(\pi-\omega) \cos \omega) \log z_{+} \\
& -\cos \omega \log \bar{z}_{+}-\cos \omega+(\pi-\omega) \sin \omega .
\end{aligned}
$$

Proof. Suppose that the solution $(v, w)=\left(v^{-2}, w^{-2}\right)$ to (56) for $k=-2$ writes

$$
\left\{\begin{array}{l}
v(z)=a \log ^{2} z+a^{\prime} \log ^{2} \bar{z}+b \log z-z^{-1} \bar{z} \\
w\left(z_{+}\right)=a \log ^{2} z_{+}+a^{\prime} \log ^{2} \bar{z}_{+}+\tilde{b} \log z_{+}+b^{\prime} \log \bar{z}_{+}+c
\end{array}\right.
$$

Then

$$
\left\{\begin{array} { l } 
{ \partial _ { z } v = 2 a z ^ { - 1 } \operatorname { l o g } z + b z ^ { - 1 } + z ^ { - 2 } \overline { z } , } \\
{ \partial _ { z _ { + } } w = 2 a z _ { + } ^ { - 1 } \operatorname { l o g } z _ { + } + \tilde { b } z _ { + } ^ { - 1 } , }
\end{array} \quad \left\{\begin{array}{l}
\partial_{\bar{z}} v=2 a^{\prime} \bar{z}^{-1} \log \bar{z}-z^{-1}, \\
\partial_{\bar{z}_{+}} w=2 a^{\prime} \bar{z}_{+}^{-1} \log \bar{z}_{+}+b^{\prime} \bar{z}_{+}^{-1}
\end{array}\right.\right.
$$


Writing the jump condition $z \partial_{z} v-\bar{z} \partial_{\bar{z}} v-z_{+} \partial_{z_{+}} w+\bar{z}_{+} \partial_{\bar{z}_{+}} w=0$ on $\theta= \pm \omega / 2$, and since $z_{+}=z e^{\mp i \pi}$ we infer the system

$$
\left\{\begin{array}{l}
2 \mathrm{i} \pi\left(a+a^{\prime}\right)+\left(b-\tilde{b}+b^{\prime}\right)=-2 e^{-\mathrm{i} \omega}, \\
-2 \mathrm{i} \pi\left(a+a^{\prime}\right)+\left(b-\tilde{b}+b^{\prime}\right)=-2 e^{\mathrm{i} \omega} .
\end{array}\right.
$$

The continuity across $\mathcal{G}$ implies:

$$
\begin{aligned}
& \pm 2 \mathrm{i} \pi\left(a-a^{\prime}\right)+\left(b-\tilde{b}-b^{\prime}\right)=0, \\
& c-\pi^{2}\left(a+a^{\prime}\right)+\pi \omega\left(a+a^{\prime}\right) \mp \mathrm{i} \pi\left(\tilde{b}-b^{\prime}\right) \mp \mathrm{i} \frac{\omega}{2}\left(b+b^{\prime}-\tilde{b}\right)+e^{\mp i \omega}=0,
\end{aligned}
$$

hence

$$
a=a^{\prime}=\frac{1}{2 \pi} \sin \omega, \quad b^{\prime}=-\cos \omega
$$

and then

$$
c=-\cos (\omega)+(\pi-\omega) \sin \omega, \quad b=-\frac{1}{\pi} \sin \omega+\frac{\omega-2 \pi}{\pi} \cos \omega, \quad \tilde{b}=-\frac{1}{\pi} \sin \omega+\frac{\omega-\pi}{\pi} \cos \omega .
$$

In order to obtain explicit formulas of any first shadow terms, it remains to derive the shadow of $\log z$. The following proposition can be checked through straightforward calculations.

Proposition 4.6. A particular solution $\mathrm{k}_{1}^{0}=\left(\zeta_{1}^{0}, \eta_{1}^{0}\right)$ to (53) with the source term equal to $-1 /(2 \pi) \log z$ (i.e. $k=0, j=1$ ) writes

$$
\begin{aligned}
-2 \pi \zeta_{1}^{0}(z) & =\frac{\sin \omega}{4 \pi} z^{2} \log ^{2} z+\frac{\sin \omega}{4 \pi} \bar{z}^{2} \log ^{2} \bar{z} \\
& -\frac{\sin \omega+2 \pi \cos \omega}{4 \pi} z^{2} \log z-\frac{3 \sin \omega+2(\pi-\omega) \cos \omega}{4 \pi} \bar{z}^{2} \log \bar{z} \\
& -\frac{1}{4}(\pi \sin \omega-\cos \omega) z^{2}+\bar{z} z \log z-z \bar{z}, \\
-2 \pi \eta_{1}^{0}\left(z_{+}\right) & =\frac{\sin \omega}{4 \pi} z_{+}^{2} \log ^{2} z_{+}+\frac{\sin \omega}{4 \pi} \bar{z}_{+}^{2} \log ^{2} \bar{z}_{+} \\
& -\frac{\sin \omega}{4 \pi} z_{+}^{2} \log z_{+}-\frac{3 \sin \omega-2 \omega \cos \omega}{4 \pi} \bar{z}_{+}^{2} \log \bar{z}_{+} \\
& -\frac{1}{4}(3 \cos \omega+(2 \omega-\pi) \sin \omega) \bar{z}_{+}^{2} .
\end{aligned}
$$

\subsection{Explicit expressions of the first shadows of primal singularities.}

To obtain the expressions of the first shadows of the primal leading singularities, we just have to take the real and imaginary parts of the functions $u^{k}$ given by Proposition 4.3, for any nonnegative integer $k \in \mathbb{N}$ :

$$
\mathfrak{s}_{1}^{k, 0}=\operatorname{Re}\left(u^{k}\right), \quad \mathfrak{s}_{1}^{k, 1}=\operatorname{Im}\left(u^{k}\right) .
$$

We recall that $\mathfrak{s}^{-}(r, \theta)$ is defined for $|\theta| \leqslant \frac{\omega}{2}$ and $\mathfrak{s}^{+}(r, \theta)$ for $|\theta| \geqslant \frac{\omega}{2}$. We also recall from (50) that $\theta_{+}=\theta-\pi \operatorname{sgn} \theta$. 
The shadow $\mathfrak{s}_{1}^{k, 0}$. For any $k \in \mathbb{N}$, the even first order shadow of $z^{k}$ writes

$$
\begin{aligned}
\mathfrak{s}_{1}^{k, 0-}(r, \theta) & =\frac{(k+1) \sin \omega+\sin (k+1) \omega}{\pi(k+1)(k+2)} r^{k+2}(\log r \cos (k+2) \theta-\theta \sin (k+2) \theta) \\
& +r^{k+2}\left(\frac{\cos k \theta}{k+1}-\frac{\cos \omega \cos (k+2) \theta}{k+2}\right), \\
\mathfrak{s}_{1}^{k, 0+}(r, \theta) & =\frac{(k+1) \sin \omega+\sin (k+1) \omega}{\pi(k+1)(k+2)} r^{k+2}\left(\log r \cos (k+2) \theta-\theta_{+} \sin (k+2) \theta\right) \\
& +r^{k+2} \frac{\cos (k+1) \omega \cos (k+2) \theta}{(k+1)(k+2)} .
\end{aligned}
$$

The shadow $\mathfrak{s}_{1}^{k, 1}$. For any $k \in \mathbb{N} \backslash\{0\}$, the odd first order shadow of $z^{k}$ writes

$$
\begin{aligned}
\mathfrak{s}_{1}^{k, 1-}(r, \theta) & =\frac{(k+1) \sin \omega-\sin (k+1) \omega}{\pi(k+1)(k+2)} r^{k+2}(\log r \sin (k+2) \theta+\theta \cos (k+2) \theta) \\
& +r^{k+2}\left(\frac{\sin k \theta}{k+1}-\frac{\cos \omega \sin (k+2) \theta}{k+2}\right), \\
\mathfrak{s}_{1}^{k, 1+}(r, \theta) & =\frac{(k+1) \sin \omega-\sin (k+1) \omega}{\pi(k+1)(k+2)} r^{k+2}(\log r \sin (k+2) \theta+\theta+\cos (k+2) \theta) \\
& -r^{k+2} \frac{\cos (k+1) \omega \sin (k+2) \theta}{(k+1)(k+2)} .
\end{aligned}
$$

\subsection{Explicit expressions of the first shadows of dual singularities}

The explicit expressions of the first shadows of the dual leading singularities are given for $k \in \mathbb{N} \backslash\{0\}$ by

$$
\mathfrak{k}_{1}^{k, 0}=\frac{1}{2 k \pi} \operatorname{Re}\left(u^{-k}\right), \quad \mathfrak{k}_{1}^{k, 1}=-\frac{1}{2 k \pi} \operatorname{Im}\left(u^{-k}\right)
$$

where the functions $u^{k}$ are given by Propositions 4.3, 4.4 and 4.5 and for $k=0$ we have

$$
\mathfrak{k}_{1}^{0,0}=\operatorname{Re}\left(k_{1}^{0}\right),
$$

where $k_{1}^{0}$ is given by Proposition 4.6.

The dual shadow $\mathfrak{k}_{1}^{k, 0}$, for $k \neq 0$.

- For $k \geqslant 3$, the even first order shadow of the dual singularity $z^{-k}$ writes

$$
\mathfrak{k}_{1}^{k, 0}=\frac{1}{2 k \pi} \mathfrak{s}_{1}^{-k, 0}
$$


where by extension, we denote by $\mathfrak{s}^{-k, 0}$ the formula (62), in which $k$ is replaced by $-k$.

- For $k=1,2$, we have

$$
\begin{aligned}
& 2 \pi \mathfrak{k}_{1}^{1,0-}(r, \theta)=\frac{\sin \omega+\omega-\pi}{\pi} r(\log r \cos \theta-\theta \sin \theta)-\cos \omega r \cos \theta+r(\log r \cos \theta+\theta \sin \theta), \\
& 2 \pi \mathfrak{k}_{1}^{1,0+}(r, \theta)=\frac{\sin \omega+\omega}{\pi} r\left(\log r \cos \theta-\theta_{+} \sin \theta\right)-r \cos \theta, \\
& 4 \pi \mathfrak{k}_{1}^{2,0-}(r, \theta)=\frac{\sin \omega}{\pi}\left(\log ^{2} r-\theta^{2}\right)-\frac{1}{\pi}(\sin \omega+(2 \pi-\omega) \cos \omega) \log r-\cos 2 \theta, \\
& 4 \pi \mathfrak{k}_{1}^{2,0+}(r, \theta)=\frac{\sin \omega}{\pi}\left(\log ^{2} r-\theta_{+}^{2}\right)-\frac{1}{\pi}(\sin \omega+(2 \pi-\omega) \cos \omega) \log r-\cos \omega+(\pi-\omega) \sin \omega .
\end{aligned}
$$

The dual shadow $\mathfrak{k}_{1}^{k, 1}$, for $k \neq 0$.

- For $k \geqslant 3$, the odd first order shadow of $z^{-k}$ writes

$$
\mathfrak{k}_{1}^{k, 1}(r, \theta)=-\frac{1}{2 k \pi} \mathfrak{s}_{1}^{-k, 1}
$$

where we denote by $\mathfrak{s}^{-k, 1}$ the formula (63), in which $k$ is replaced by $-k$.

- For $k=1,2$, we have

$$
\begin{aligned}
& -2 \pi \mathfrak{k}_{1}^{1,1-}(r, \theta)=\frac{\sin \omega-\omega+\pi}{\pi} r(\log r \sin \theta+\theta \cos \theta)-\cos \omega r \sin \theta-r(\log r \sin \theta-\theta \cos \theta), \\
& -2 \pi \mathfrak{k}_{1}^{1,1+}(r, \theta)=\frac{\sin \omega-\omega}{\pi} r\left(\log r \sin \theta+\theta_{+} \cos \theta\right)+r \sin \theta, \\
& -4 \pi \mathfrak{k}_{1}^{2,1-}(r, \theta)=-\frac{1}{\pi}(\sin \omega+(2 \pi-\omega) \cos \omega) \theta+\sin 2 \theta, \\
& -4 \pi \mathfrak{k}_{1}^{2,1+}(r, \theta)=-\frac{1}{\pi}(\sin \omega-\omega \cos \omega) \theta_{+} .
\end{aligned}
$$

The shadow $\mathfrak{k}_{1}^{0,0}$.

$$
\begin{aligned}
-2 \pi \mathfrak{k}_{1}^{0,0}-(r, \theta) & =\frac{\sin \omega}{2 \pi} r^{2}\left(\cos 2 \theta\left(\log ^{2} r-\theta^{2}\right)-2 \theta \sin 2 \theta \log r\right) \\
& +\frac{(\omega-2 \pi) \cos \omega-2 \sin \omega}{2 \pi} r^{2}(\cos 2 \theta \log r-\theta \sin 2 \theta) \\
& +\frac{\cos \omega-\pi \sin \omega}{4} r^{2} \cos 2 \theta+r^{2} \log r-r^{2} \\
-2 \pi \mathfrak{k}_{1}^{0,0+}(r, \theta) & =\frac{\sin \omega}{2 \pi} r^{2}\left(\cos 2 \theta\left(\log ^{2} r-\theta_{+}^{2}\right)-2 \theta_{+} \sin 2 \theta \log r\right) \\
& +\frac{\omega \cos \omega-2 \sin \omega}{2 \pi} r^{2}\left(\cos 2 \theta \log r-\theta_{+} \sin 2 \theta\right) \\
& -\frac{3 \cos \omega+(2 \omega-\pi) \sin \omega}{4} r^{2} \cos 2 \theta
\end{aligned}
$$

\section{Numerical simulations}

In order to illustrate the calculus of the first shadows, mostly what is proposed in Section 4, and the technique described in subsection 3.4 to compute the coefficients of expansion (21), we consider the 
following problem (problem (1) with $J=0$ except the non-homogeneous Dirichlet boundary condition)

$$
\left\{\begin{array}{rlrl}
-\Delta \mathcal{A}^{+} & =0 \text { in } \Omega_{+}, & \\
-\Delta \mathcal{A}^{-}+4 \mathrm{i} \zeta^{2} \mathcal{A}^{-} & =0 \text { in } \Omega_{-}, & {[\mathcal{A}]_{\Sigma}=0,} & \text { on } \Sigma, \\
\mathcal{A} & =\frac{|\theta|}{2 \pi} \text { on } \Gamma, & {\left[\partial_{n} \mathcal{A}\right]_{\Sigma}=0,} & \text { on } \Sigma .
\end{array}\right.
$$

Since the source term is even with respect to $\theta$, the solution $\mathcal{A}$ of (64) is $\theta$-even. As a consequence, only the terms with indices $p=0$ are involved in the Kondratev-type expansion (21). The computational domain $\Omega$, depicted in Figure 2(a), is a disk of radius $50 \mathrm{~mm}$. We consider a conducting sector for $\omega=\pi / 4$ (other values have been tested and the conclusions are similar). We particularly focus on the behavior of the solution in the vicinity of the corner c. Parameter $\zeta$ is equal to $1 /(5 \sqrt{2}) \mathrm{mm}^{-1}$, which corresponds to a physical skin depth of $5 \mathrm{~mm}$. The solution computed by the finite element method, using $P_{2}$ finite elements available in the library [7] and a mesh with 64192 triangles is plotted in Figure 2(b) for the real part and Figure 2(c) for the imaginary part.

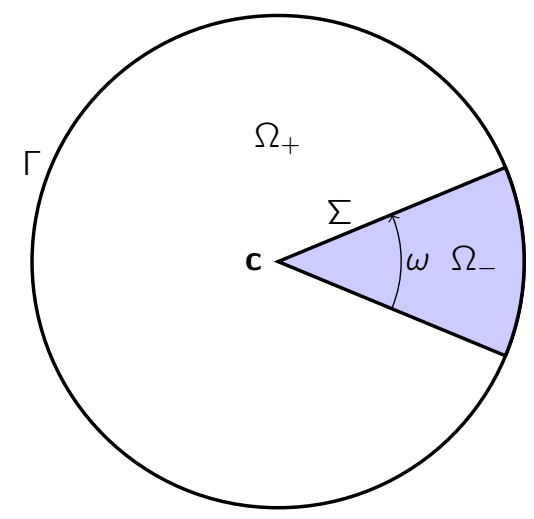

(a) Domain $\Omega$.

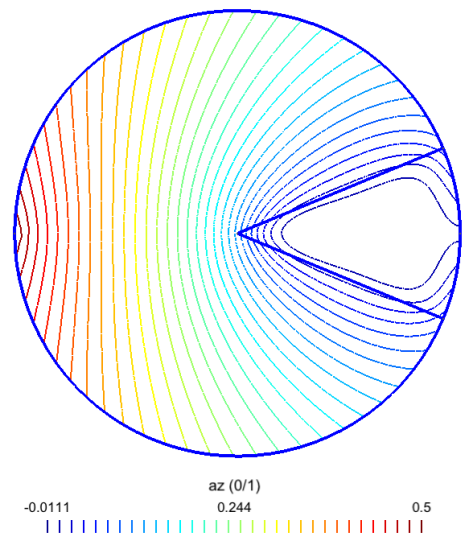

(b) Real part of the solution $\mathcal{A}$.

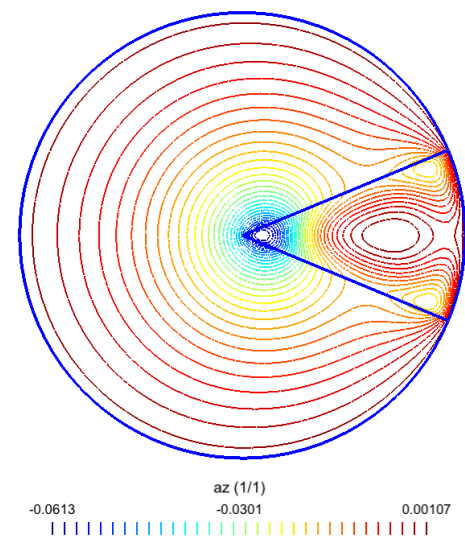

(c) Imaginary part of the solution $\mathcal{A}$.

Figure 2: Domain $\Omega$ and the computed solution for problem (64).

First, we consider the computation of $\wedge^{0,0}$ by the use of formula (35) for the case $m=0$ and $m=1$. The value of $\mathcal{A}$ computed at the corner $\mathbf{c}$ is defined as the reference value for $\wedge^{0,0}$; note that 
it is already a numerically approximated value. This reference value is compared to $\mathcal{J}_{R}\left(\mathfrak{K}_{m}^{0,0}, \mathcal{A}\right)$ for $m=0$ and $m=1$, a quantity which provides an approximate value of $\Lambda^{0,0}$ by the method described in subsection 3.4. Note that the convergence rate as a function of $R$ is related to the first neglected terms. The results are shown in Figure 3 and are consistent with the theoretical convergence rate (remind $R_{0}$ defined in (26)). Note that concerning $m=1$ and the smallest values of $R$, we can presume that the discretization accuracy is attained: This should explain the behavior for the smallest values of $R$ in Figure 3.

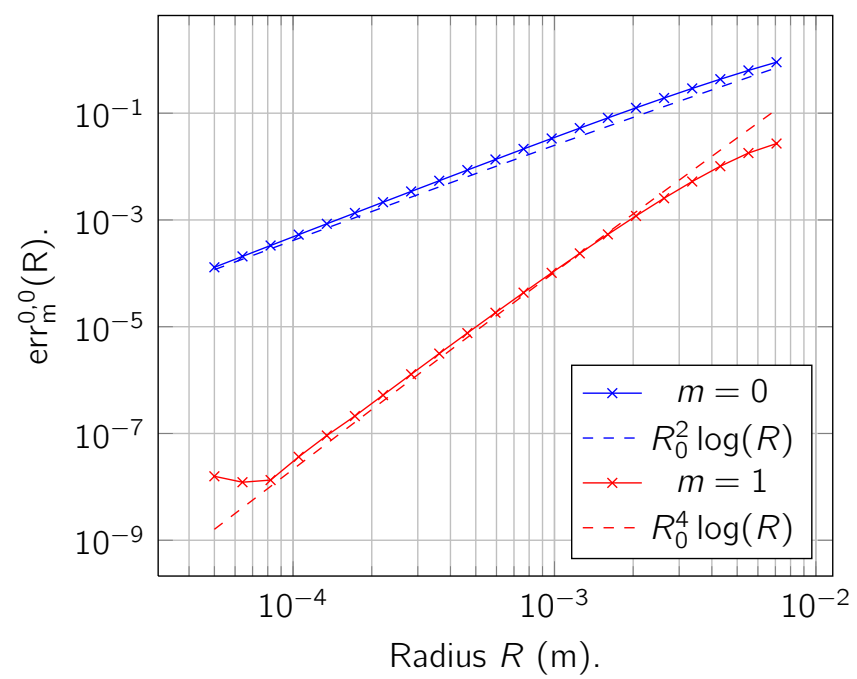

Figure 3: Accuracy for the computation of $\Lambda^{0,0}$ as a function of $R$. Quantity $\operatorname{err}_{\mathrm{m}}^{0,0}(\mathrm{R})$ is the relative error $\mid \mathcal{J}_{R}\left(\mathfrak{K}_{m}^{0,0}, \mathcal{A}\right)-$ $\mathcal{A}(\mathbf{c})|/| \mathcal{A}(\mathbf{c}) \mid$.

Reference value $\mathcal{A}(\mathbf{c}):(0.114449904-\mathrm{i} 0.0464907336)$.

Second, we also consider the computation of the coefficients $\Lambda^{1,0}$ and $\Lambda^{2,0}$. The reference values are taken from the computation of $\mathcal{J}_{R}$ for the small value $R_{\text {small }}=5 \cdot 10^{-5} \mathrm{~m}$ of $R$. Results for $\mathcal{J}_{R}\left(\mathfrak{K}_{m}^{1,0}, \mathcal{A}\right), m=0,1$ are shown in Figure 4; they are also consistent with the expected theoretical behaviors. Results for $\mathcal{J}_{R}\left(\mathfrak{K}_{1}^{2,0}, \mathcal{A}\right)$ are shown in Figure 5. In order to deduce from $\mathcal{J}_{R}\left(\mathfrak{K}_{1}^{2,0}, \mathcal{A}\right)$ the approximate value of $\Lambda^{2,0}$, we have to use the computed value for $\Lambda^{0,0}$ and the coefficient $\mathcal{J}^{2,0 ; 0,0}$. This last coefficient can be obtained from Proposition 3.7:

$$
\mathcal{J}^{2,0 ; 0,0}=i \zeta^{2} \int_{0}^{2 \pi} \Psi_{0,0}^{2,0}(\theta)\left[4 \Phi_{1,0}^{0,0}(\theta)+\Phi_{1,1}^{0,0}(\theta)\right]-\Psi_{1,1}^{2,0}(\theta) \Phi_{0,0}^{0,0}(\theta) d \theta
$$

Angular functions $\Phi_{0,0}^{0,0}, \Phi_{1,0}^{0,0}$ and $\Phi_{1,1}^{0,0}$ can be identified by comparing (18) and (22) when $k=0, p=0$, and $j \in\{0,1\}$

$$
\mathfrak{s}_{j}^{0,0}(r, \theta)=r^{2 j} \sum_{n=0}^{j} \log ^{n} r \Phi_{j, n}^{0,0}(\theta)
$$

RR n ${ }^{\circ} 8204$ 
and using $\mathfrak{s}_{0}^{0,0}(r, \theta)=1(5)$ and the expression of $\mathfrak{s}_{1}^{0,0}(62)$

$\Phi_{0,0}^{0,0}(\theta)=1, \quad \Phi_{1,1}^{0,0}(\theta)=\frac{\sin \omega}{\pi} \cos 2 \theta, \quad \Phi_{1,0}^{0,0}(\theta)= \begin{cases}1-\frac{\cos \omega}{2} \cos 2 \theta-\frac{\sin \omega}{\pi} \theta \sin 2 \theta & \text { for } \quad|\theta| \leqslant \frac{\omega}{2}, \\ \frac{\cos \omega}{2} \cos 2 \theta-\frac{\sin \omega}{\pi} \theta_{+} \sin 2 \theta & \text { for } \quad|\theta| \geqslant \frac{\omega}{2} .\end{cases}$

Angular functions $\Psi_{0,0}^{2,0}$ and $\Psi_{1,1}^{2,0}$ can be identified by comparing (23) and (25) when $k=2, p=0$ and using (7) and $\mathfrak{k}_{1}^{2,0}$ from Subsection 4.4

$$
\Psi_{0,0}^{2,0}(\theta)=\frac{1}{4 \pi} \cos 2 \theta, \quad \Psi_{1,1}^{2,0}(\theta)=-\frac{1}{4 \pi^{2}}(\sin \omega+(2 \pi-\omega) \cos \omega) .
$$

Computing integrals, we obtain

$$
\mathcal{J}^{2,0 ; 0,0}=i \zeta^{2}\left(\frac{3 \sqrt{2}}{4 \pi}+\frac{5 \sqrt{2}}{8}\right) \approx i \zeta^{2} 1.221502
$$

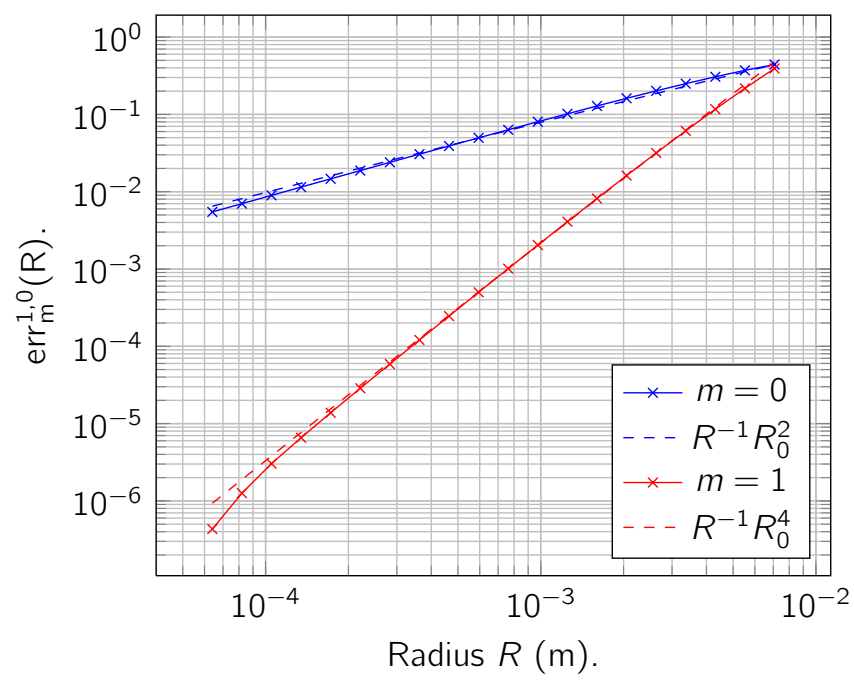

Figure 4: Accuracy for the computation of $\Lambda^{1,0}$ as a function of $R$.

Quantity $\operatorname{err}_{m}^{1,0}(\mathrm{R})$ stands for the relative error $\mid \mathcal{J}_{R}\left(\mathfrak{K}_{m}^{1,0}, \mathcal{A}\right)$ $\mathcal{J}_{R_{\text {small }}}\left(\mathfrak{K}_{1}^{1,0}, \mathcal{A}\right)|/| \mathcal{J}_{R_{\text {small }}}\left(\mathfrak{K}_{1}^{1,0}, \mathcal{A}\right) \mid$.

Reference value $\mathcal{J}_{R_{\text {small }}}\left(\mathfrak{K}_{1}^{1,0}, \mathcal{A}\right):(-12.970664-i 5.40915055)$. 


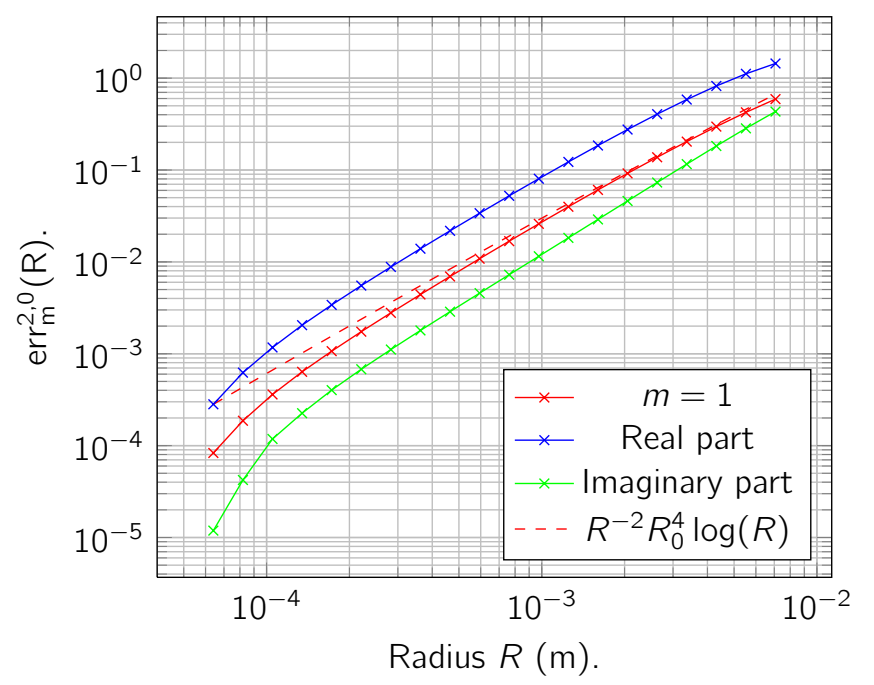

Figure 5: Accuracy for the computation of $\wedge^{2,0}$ as a function of $R$.

Quantity $\operatorname{err}_{\mathrm{m}}^{2,0}(\mathrm{R})$ stands for the relative error of the real part, of the imaginary part and of the modulus of $\left(\mathcal{J}_{R}\left(\mathfrak{K}_{1}^{2,0}, \mathcal{A}\right)-\mathcal{J}_{R_{\text {small }}}\left(\mathfrak{K}_{1}^{2,0}, \mathcal{A}\right)\right)$.

Reference value $\mathcal{J}_{R_{\text {small }}}\left(\mathfrak{K}_{1}^{2,0}, \mathcal{A}\right):(1406.54919+\mathrm{i} 4599.19999)$. 
In Figure 6, we perform a qualitative description of the isovalues of $\mathcal{A}$ close to the corner comparing the finite element solution and, successively,

- expansion (21) restricted to a composite order 1, i.e.

$$
\mathcal{J}_{R_{\text {small }}}\left(\mathfrak{K}_{1}^{0,0}, \mathcal{A}\right)+\mathcal{J}_{R_{\text {small }}}\left(\mathfrak{K}_{1}^{1,0}, \mathcal{A}\right) \mathfrak{s}_{0}^{1,0},
$$

- expansion (21) restricted to a composite order 2, i.e.

$$
\mathcal{J}_{R_{\text {small }}}\left(\mathfrak{K}_{1}^{0,0}, \mathcal{A}\right)\left(1+i \zeta^{2} \mathfrak{s}_{1}^{0,0}\right)+\mathcal{J}_{R_{\text {small }}}\left(\mathfrak{K}_{1}^{1,0}, \mathcal{A}\right) \mathfrak{s}_{0}^{1,0}+\left(\mathcal{J}_{R_{\text {small }}}\left(\mathfrak{K}_{1}^{2,0}, \mathcal{A}\right)-\mathcal{J}^{2,0 ; 0,0} \mathcal{J}_{R_{\text {small }}}\left(\mathfrak{K}_{1}^{0,0}, \mathcal{A}\right)\right) \mathfrak{s}_{0}^{2,0}
$$

- expansion (21) restricted to a composite order 3, i.e. adding to the expression above the term

$$
\left(\mathcal{J}_{R_{\text {small }}}\left(\mathfrak{K}_{1}^{3,0}, \mathcal{A}\right)-\mathcal{J}^{3,0 ; 1,0} \mathcal{J}_{R_{\text {small }}}\left(\mathfrak{K}_{1}^{1,0}, \mathcal{A}\right)\right) \mathfrak{s}_{0}^{3,0},
$$

and replacing $\mathfrak{s}_{0}^{1,0}$ by $\mathfrak{s}_{0}^{1,0}+i \zeta^{2} \mathfrak{s}_{1}^{1,0}$. Computing integrals, we obtain

$$
\mathcal{J}^{3,0 ; 1,0} \approx \mathrm{i} \zeta^{2} 1.522117 \cdot 10^{-9}
$$

The reference value for $\mathcal{J}_{R_{\text {small }}}\left(\mathfrak{K}_{1}^{3,0}, \mathcal{A}\right)$ is $(93037.6253-\mathrm{i} 154720.669)$.

For the composite order 1, only the constant and linear terms with respect to $r$ are collected in the Kondratev-type expansion. For the composite order 2, the terms which behave as $r^{2}$ and $r^{2} \log r$ are added. Adding then the terms which behave as $r^{3}$ and $r^{3} \log r$ leads to the composite order 3 .

Both on the real and imaginary parts, we observe in Figure 6 as expected that the increase of the order enables to increase the accuracy.

\section{Conclusion}

In this paper, we have provided corner asymptotics of the magnetic potential for the eddy current problem in a bidimensional domain. Such expansions involve two ingredients: The calculation of both primal and dual singularities, and the computation of the singular coefficients. Primal and dual singularities of the non-homogeneous operator $-\Delta+i \kappa \mu_{0} \sigma \mathbb{1}_{\Omega_{-}}$in $\mathbb{R}^{2}$ are derived as infinite series, whose coefficients are obtained recursively. To compute the singular coefficients, we first tried to apply the method of moments, straightforwardly derived from the case of the Laplace operator. This method is limited since it makes possible to obtain only the first singular coefficients of the corner asymptotics and at a low order of accuracy, therefore we adapted the method of quasi-dual functions introduced in [3] to our specific problem. Numerical simulations of section 5 corroborate the theoretical order of accuracy obtained in the previous sections, illustrating the accuracy of the asymptotics.

Forthcoming work will deal with the high conducting case, (I.e. the case $\sigma$ goes to infinity), for which two small parameters appear: the distance to the corner and the skin depth $\sqrt{2 /\left(\kappa \mu_{0} \sigma\right)}$. Preliminary results of the formal derivation of the magnetic potential have been obtained by Buret et al in [1], which have to be rigorously justified and extended. 


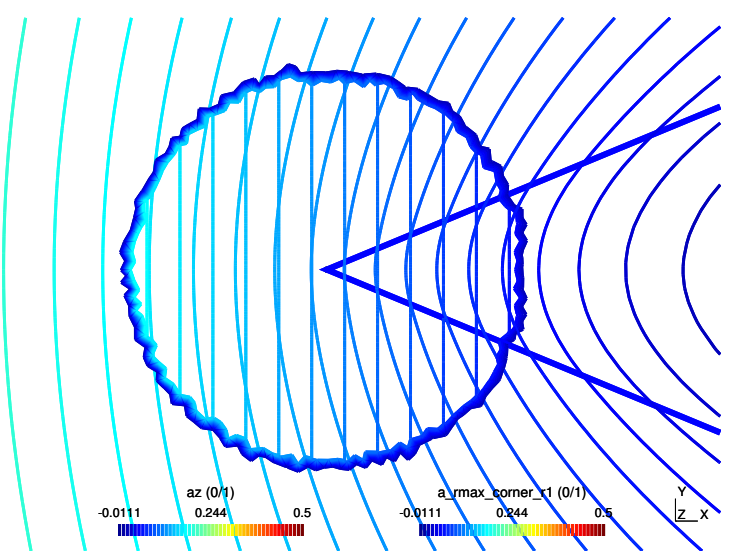

(a) Expansion restricted to composite order 1. Real part.

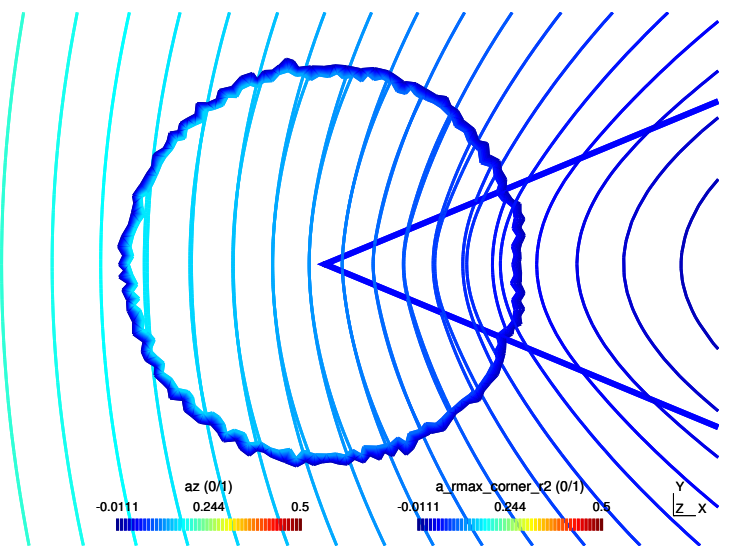

(c) Expansion restricted to composite order 2. Real part.

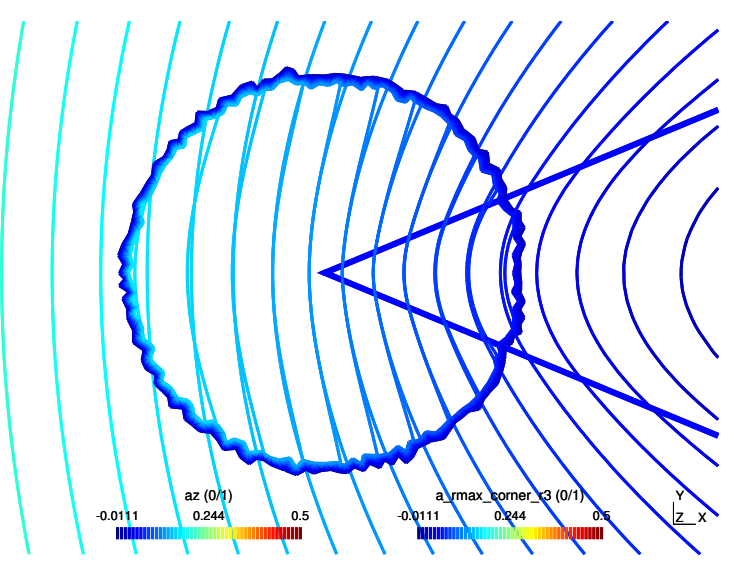

(e) Expansion restricted to composite order 3. Real part.

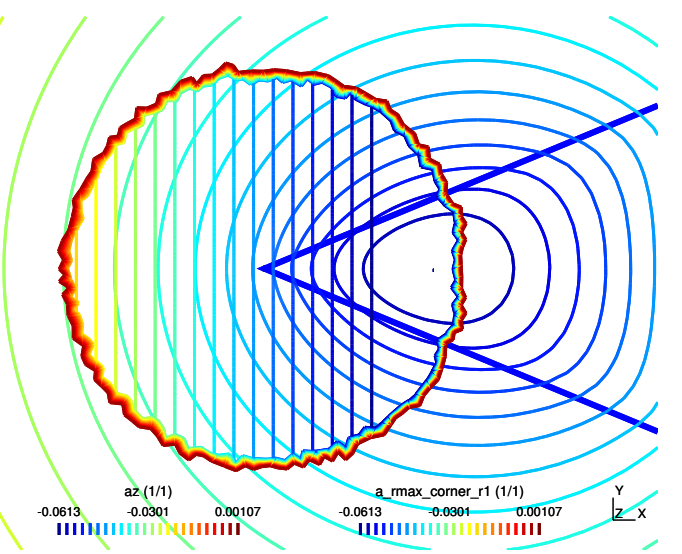

(b) Expansion restricted to composite order 1. Imaginary part.

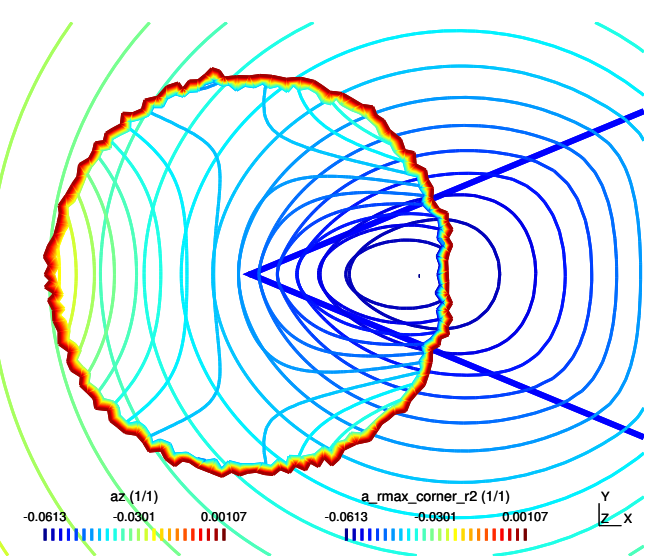

(d) Expansion restricted to composite order 2. Imaginary part.

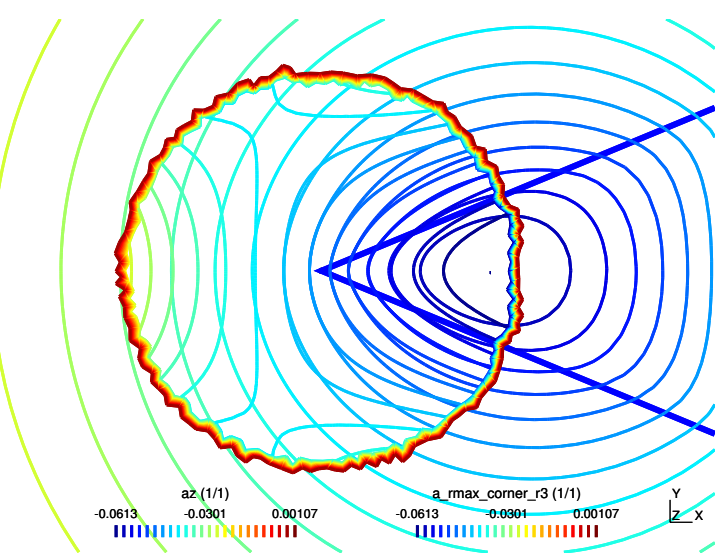

(f) Expansion restricted to composite order 3. Imaginary part.

Figure 6: Comparison of the finite element solution and of the local expansion. 


\section{References}

[1] François Buret, Monique Dauge, Patrick Dular, Laurent Krähenbühl, Victor Péron, Ronan Perrussel, Clair Poignard, and Damien Voyer. Eddy currents and corner singularities. IEEE Trans. on Mag., 48(2):679-682, 2012.

[2] M. Costabel and M. Dauge. Construction of corner singularities for Agmon-Douglis-Nirenberg elliptic systems. Math. Nachr., 162:209-237, 1993.

[3] M. Costabel, M. Dauge, and Z. Yosibash. A quasidual function method for extracting edge stress intensity functions. SIAM Jour. Math. Anal., 35(5):1177-1202, 2004.

[4] M. Dauge, S. Nicaise, M. Bourlard, and J. Mbaro-Saman Lubuma. Coefficients des singularités pour des problèmes aux limites elliptiques sur un domaine à points coniques. II. Quelques opérateurs particuliers. RAIRO Modél. Math. Anal. Numér., 24(3):343-367, 1990.

[5] Monique Dauge. Elliptic Boundary Value Problems in Corner Domains - Smoothness and Asymptotics of Solutions. Lecture Notes in Mathematics, Vol. 1341. Springer-Verlag, Berlin, 1988.

[6] E.M. Deeley. Surface impedance near edges and corners in three-dimensional media. IEEE Trans. on Mag., 26(2):712-714, 1990.

[7] P. Dular and C. Geuzaine. Getdp: a general environment for the treatment of discrete problems. Source code. http://geuz.org/getdp/, 1997-2012.

[8] P. Grisvard. Boundary Value Problems in Non-Smooth Domains. Pitman, London, 1985.

[9] H. Haddar, P. Joly, and H.-M. Nguyen. Generalized impedance boundary conditions for scattering problems from strongly absorbing obstacles: the case of Maxwell's equations. Math. Models Methods Appl. Sci., 18(10):1787-1827, 2008.

[10] V. A. Kondratev. Boundary value problems for elliptic equations in domains with conical or angular points. Trudy Moskov. Mat. Obšč., 16:209-292, 1967.

[11] V. A. Kondratev and O. A. Oleinik. Boundary-value problems for partial differential equations in non-smooth domains. Russian Math. Surveys, 38:1-86, 1983.

[12] V. A. Kozlov, V. G. Maz'ya, and J. Rossmann. Elliptic boundary value problems in domains with point singularities. Mathematical Surveys and Monographs, 52. American Mathematical Society, Providence, RI, 1997.

[13] M. A. Leontovich. Approximate boundary conditions for the electromagnetic field on the surface of a good conductor. In Investigations on radiowave propagation, volume 2, pages 5-12. Printing House of the USSR Academy of Sciences, Moscow, 1948.

[14] V. G. Maz'ya and B. A. Plamenevskii. On the coefficients in the asymptotic of solutions of the elliptic boundary problem in domains with conical points. Amer. Math. Soc. Trans. (2), 123:57-88, 1984.

[15] M.-A. Moussaoui. Sur l'approximation des solutions du problème de Dirichlet dans un ouvert avec coins. In Singularities and constructive methods for their treatment (Oberwolfach, 1983), volume 1121 of Lecture Notes in Math., pages 199-206. Springer, Berlin, 1985. 
[16] Serge Nicaise. Polygonal interface problems. Methoden und Verfahren der Mathematischen Physik, 39. Verlag Peter D. Lang, Frankfurt-am-Main, 1993.

[17] S. M. Rytov. Calcul du skin effect par la méthode des perturbations. Journal of Physics, 11(3):233-242, 1940.

[18] T. B. A. Senior and J. L. Volakis. Approximate Boundary Conditions in Electromagnetics. IEE Electromagnetic Waves Series, 41. London : Institution of Electrical Engineers, Royaume-Uni, 1995.

[19] S. Shannon, Z. Yosibash, M. Dauge, and M. Costabel. Extracting generalized edge flux intensity functions by the quasidual function method along circular 3-d edges. Preprint HAL available at http://hal .archives-ouvertes.fr/hal-00725928., 2012.

[20] Barna A. Szabó and Zohar Yosibash. Numerical analysis of singularities in two dimensions. II. Computation of generalized flux/stress intensity factors. Internat. J. Numer. Methods Engrg., 39(3):409-434, 1996.

[21] S. Yuferev, L. Proekt, and N. Ida. Surface impedance boundary conditions near corner and edges: Rigorous consideration. IEEE Trans. on Mag., 37(5):3465-3468, 2001.

\section{A Appendix: Tools for the derivation of the shadows at any order}

As described previously, the singularities of the operator $\mathscr{L}_{\zeta}(13)$ are obtained as series involving the shadows of the leading singularities at any order. Moreover, these shadows can be described with the help of the complex variable Ansatz introduced in Section 4 and spaces $\mathbb{S}_{\ell, n}^{\lambda}$ defined by equations (55). However, the derivation of a generic formula for the shadows is hardly (and tediously!) reachable, because of the growth of the number of the terms with the order of the shadow.

For instance, the leading singularity $z \in \mathbb{S}_{0,0}^{1}$ generates a primal shadow $s_{1}^{1} \in \mathbb{S}_{1,1}^{3}$. According to Proposition 4.3, this shadow $s_{1}^{1}$ contains 4 terms: $z^{3} \log z, \bar{z}^{3} \log \bar{z}, z^{3}$ and $z^{2} \bar{z}$ in the sector $\mathcal{S}_{-}$, and we shall prove that each of these terms generates a shadow belonging to $\mathbb{S}_{2,2}^{5}$. This shows the complexity of the generic expression of the singularities. Nevertheless, it is possible to obtain the expansion of the singularities at any order using a formal calculus tool. The aim of this section is to provide the elementary results in order to derive the singularities, after appropriate use of a formal calculus algorithm.

Actually, we see that, generally speaking, the shadow of the $k^{\text {th }}$ leading singularity at the order $j$ is a combination of functions of the form $z^{k+2 j-\ell} \bar{z}^{\ell} \log ^{n} z$ (or its conjugate), where $(n, \ell) \in \mathbb{N}^{2}$ and $(k, j) \in \mathbb{Z} \times \mathbb{N}$. Therefore, if we provide a way to obtain the shadow of this generic function, it will be possible to assemble the terms in order to obtain the full expression of the singularities.

For any $(\lambda, \ell, n) \in \mathbb{Z} \times \mathbb{N}^{2}$, the idea of the derivation of the shadow generated by $z^{\lambda-\ell} \bar{Z}^{\ell} \log ^{n} z$ consists in performing the same calculus as for the first order shadows, by only considering the terms of higher degree in $\log z$, the remainder terms being neglected, and treated then at the next iteration. The principle of such calculations holds behind the calculus of Section 4.

A congruence relation on the spaces $\mathbb{S}_{\ell, n}^{\lambda}$ is thus needed. Moreover, due to the omission of the terms of lower degree, errors are generated on the boundary $\mathcal{G}$, which have to be corrected and therefore appropriate spaces of traces and a congruence on such spaces have also to be given. 


\section{A.1 Definition of the space $\mathbb{J}_{n}^{\lambda}$ on $\mathcal{G}$ and the congruence relation $\equiv$ on $\mathbb{S}_{\ell, n}^{\lambda}$ and $\mathbb{J}_{n}^{\lambda}$}

Definition A.1. For $(\lambda, n) \in \mathbb{Z} \times \mathbb{N}$ denote by $\mathbb{J}_{n}^{\lambda}$ the space

$$
\mathbb{J}_{n}^{\lambda}=\left\{g: \quad \forall z \in \mathcal{G}, g(z)=z^{\lambda} \sum_{q=0}^{n} \log ^{q} z\left\{\begin{array}{ll}
\alpha_{q}, & \text { if } \arg z=\omega / 2 \\
\overline{\alpha_{q}}, & \text { if } \arg z=-\omega / 2
\end{array}, \quad\left(\alpha_{q}\right)_{q=0}^{n} \in \mathbb{C}^{n+1}\right\} .\right.
$$

Remark A.2. We would obtain the same space $\mathbb{J}_{n}^{\lambda}$ if we replace everywhere $z^{\lambda} \log ^{q} z$ in (66) by $z^{\lambda} \log ^{q} \bar{z}$. The same holds with $\bar{z}^{\lambda} \log ^{q} z$, or $\bar{z}^{\lambda} \log ^{q} \bar{z}$, instead of $z^{\lambda} \log ^{q} z$.

Definition A.3. The congruence relation on $\mathbb{S}_{\ell, n}^{\lambda}(55)$ and $\mathbb{J}_{n}^{\lambda}(66)$ is defined as follows:

$$
\forall(\lambda, \ell, n) \in \mathbb{Z} \times \mathbb{N} \times \mathbb{N} \backslash\{0\}, \quad\left\{\begin{array}{llll}
\forall u, u^{\prime} \in \mathbb{S}_{\ell, n}^{\lambda}, & u \equiv u^{\prime} & \text { iff } & u-u^{\prime} \in \mathbb{S}_{\ell, n-1}^{\lambda}, \\
\forall g, g^{\prime} \in \mathbb{J}_{n}^{\lambda}, & g \equiv g^{\prime} & \text { iff } & g-g^{\prime} \in \mathbb{J}_{n-1}^{\lambda},
\end{array}\right.
$$

and for $n=0$ we define $\equiv$ as the usual equality between two functions.

Roughly speaking, the congruence relation consists in identifying two functions with the same coefficient in front of the highest power of the logarithmic terms.

\section{A.2 Generic elementary calculation}

Throughout this subsection, we choose an integer $\lambda \in \mathbb{Z}$ (which corresponds to the degree of homogeneity of the solution) and a natural number $\ell \in \mathbb{N}$. Define the sequences of functions of the complex variables $\left(f_{n}^{*}, g_{n}^{*}, h_{n}^{*}\right)_{n \in \mathbb{N}}$ as

$$
\forall n \in \mathbb{N}, \quad \begin{cases}\forall z \in \mathcal{S}_{-}, & f_{n}^{*}(z)=z^{\lambda-2-\ell} \bar{z}^{\ell} \log ^{n} z, \\ \forall z \in \mathcal{G}, & g_{n}^{*}(z)=z^{\lambda-1} \log ^{n} z, \\ \forall z \in \mathcal{G}, & h_{n}^{*}(z)=\bar{z}^{\lambda-1} \log ^{n} \bar{z} .\end{cases}
$$

According to the previous observations, the primal and dual shadow terms at any order are generated by linear combinations of such $\left(f_{n}^{*}, g_{n}^{*}, h_{n}^{*}\right)$. For any $n \in \mathbb{N}$, we also denote by $v_{n}^{*}$ the function

$$
\forall z \in \mathcal{S}_{-}, \quad v_{n}^{*}(z)= \begin{cases}\frac{z^{\lambda-\ell-1}}{\lambda-\ell-1} \frac{\bar{z}^{\ell+1}}{\ell+1} \log ^{n} z, & \text { if } \ell \neq \lambda-1, \\ \frac{1}{n+1} \frac{\bar{z}^{\ell+1}}{\ell+1} \log ^{n+1} z, & \text { if } \ell=\lambda-1 .\end{cases}
$$

Now we choose $n \geqslant 1$ and investigate an induction step. Let $\left(\alpha_{n}, \beta_{n}, \gamma_{n}\right) \in \mathbb{R} \times \mathbb{C}^{2}$, and let $\left(f_{n}, g_{n}, h_{n}\right)$ be defined by

$$
\begin{aligned}
& \forall z \in \mathcal{S}_{-}, \quad f_{n}(z)=\alpha_{n} f_{n}^{*}, \\
& \forall z \in \mathcal{G}, \quad g_{n}(z)= \begin{cases}\beta_{n} g_{n}^{*}(z), & \text { for } \theta=\omega / 2, \\
\overline{\beta_{n}} g_{n}^{*}(z), & \text { for } \theta=-\omega / 2,\end{cases} \\
& \forall z \in \mathcal{G}, \quad h_{n}(z)= \begin{cases}\gamma_{n} h_{n}^{*}(z), & \text { for } \theta=\omega / 2, \\
\overline{\gamma_{n}} h_{n}^{*}(z), & \text { for } \theta=-\omega / 2 .\end{cases}
\end{aligned}
$$

Therefore,

$$
\left(f_{n}, g_{n}, h_{n}\right) \in \mathbb{S}_{\ell, n}^{\lambda-2,-} \times \mathbb{J}_{n}^{\lambda-1} \times \mathbb{J}_{n}^{\lambda-1} .
$$


We are going to show that the shadow term $\left(V_{n}, W_{n}\right)$ generated by the triple $\left(f_{n}, g_{n}, h_{n}\right)$ can be obtained recursively in $\mathbb{S}_{\ell+1, n+1}^{\lambda}$ when $\lambda \neq 0$, and in $\mathbb{S}_{\ell+1, n+2}^{0}$ when $\lambda=0$ by solving a partial differential equation problem, thanks to the congruence relation $\equiv$. Actually, the shadow $\left(V_{n}, W_{n}\right)$ generated by $\left(f_{n}, g_{n}, h_{n}\right)$ satisfies

$$
\left\{\begin{aligned}
\partial_{z} \partial_{\bar{z}} V_{n} & =f_{n}, & & \text { in } \mathcal{S}_{-}, \\
\partial_{z_{+}} \partial_{\bar{z}_{+}} W_{n} & =0, & & \text { in } \mathcal{S}_{+} \\
\partial_{z} V_{n}+\partial_{z_{+}} W_{n} & =g_{n}, & & \text { on } \mathcal{G} \\
\partial_{\bar{z}} V_{n}+\partial_{\bar{z}_{+}} W_{n} & =h_{n}, & & \text { on } \mathcal{G}
\end{aligned}\right.
$$

Throughout this subsection, in order to simplify the notations, we set

$$
\ell^{\prime}=\lambda-2-\ell, \quad m=\lambda-1 .
$$

\section{A.2.1 The case $\lambda \neq 0$}

We first consider the case $\lambda \neq 0$, which corresponds to a source term which does not belong to $\mathrm{T}^{-2}$, with the notation of Lemma 3.1.

Proposition A.4. Let $(\lambda, \ell, n) \in \mathbb{Z} \times \mathbb{N}^{2}$ and let $\left(f_{n}, g_{n}, h_{n}\right)$ be defined by (69). We suppose that $\lambda \neq 0$ and

$$
\text { either }(\ell \neq \lambda-1) \text {, or }\left(\ell=\lambda-1 \text { and } \alpha_{n}=0\right) \text {. }
$$

We define $\left(v_{n}, w_{n}\right)$ as

$$
\begin{cases}v_{n}(z) & =a z^{\lambda} \log ^{n+1} z+a^{\prime} \bar{z}^{\lambda} \log ^{n+1} \bar{z}+b z^{\lambda} \log ^{n} z, \\ (-1)^{\lambda} w_{n}\left(z_{+}\right) & =a z_{+}^{\lambda} \log ^{n+1} z_{+}+a^{\prime} \bar{z}_{+}^{\lambda} \log ^{n+1} \bar{z}_{+}+b^{\prime} \bar{z}_{+}^{\lambda} \log ^{n} \bar{z}_{+},\end{cases}
$$

where, using notations (71)

$$
\left\{\begin{array}{l}
a=\frac{1}{\pi \lambda(n+1)}\left(\operatorname{Im} \beta_{n}+\alpha_{n} \frac{\sin \omega(\ell+1)}{(\ell+1)}\right) \\
b=\frac{1}{\lambda}\left(\operatorname{Re} \beta_{n}-\alpha_{n} \frac{\cos \omega(\ell+1)}{(\ell+1)}\right)
\end{array},\left\{\begin{array}{l}
a^{\prime}=\frac{1}{\pi \lambda(n+1)}\left(-\operatorname{Im} \gamma_{n}+\alpha_{n} \frac{\sin \omega\left(\ell^{\prime}+1\right)}{\left(\ell^{\prime}+1\right)}\right) \\
b^{\prime}=\frac{1}{\lambda}\left(-\operatorname{Re} \gamma_{n}+\alpha_{n} \frac{\cos \omega\left(\ell^{\prime}+1\right)}{\left(\ell^{\prime}+1\right)}\right)
\end{array}\right.\right.
$$

Then, $\left(\alpha_{n} v_{n}^{*}+v_{n}, w_{n}\right)$ is a particular solution to (70) in $\mathbb{S}_{\ell+1, n+1}^{\lambda}$ for the congruence relation $\equiv$. This means that the couple of functions $\left(V_{n-1}, W_{n-1}\right)$ defined by

$$
V_{n-1}=V_{n}-\left(\alpha_{n} v_{n}^{*}+v_{n}\right), \quad W_{n-1}=W_{n}-w_{n}
$$

satisfies the following problem:

$$
\begin{cases}\partial_{z} \partial_{\bar{z}} V_{n-1}=f_{n-1}, & \text { in } \mathcal{S}_{-}, \\ \partial_{z_{+}} \partial_{\bar{z}_{+}} W_{n-1}=0, & \text { in } \mathcal{S}_{+}, \\ \partial_{z} V_{n-1}+\partial_{z_{+}} W_{n-1}=g_{n-1}, & \text { on } \mathcal{G} \\ \partial_{\bar{z}} V_{n-1}+\partial_{\bar{z}_{+}} W_{n-1}=h_{n-1}, & \text { on } \mathcal{G},\end{cases}
$$

where

$$
\left(f_{n-1}, g_{n-1}, h_{n-1}\right) \in \mathbb{S}_{\ell, n-1}^{\lambda-2,-} \times \mathbb{J}_{n-1}^{\lambda-1} \times \mathbb{J}_{n-1}^{\lambda-1} .
$$


More precisely we have the explicit formulas

$$
\begin{aligned}
f_{n-1} & =-\frac{n \alpha_{n}}{\ell^{\prime}+1} f_{n-1}^{*}, \\
g_{n-1} & =n(b-a(n+1)((\mp \mathrm{i} \pi) \lambda / 2+1)(\mp \mathrm{i} \pi)) g_{n-1}^{*} \\
& -a z^{m} \sum_{q=0}^{n-2}\left((\mp \mathrm{i} \pi) \lambda\left(\begin{array}{c}
q \\
n+1
\end{array}\right)+(n+1)\left(\begin{array}{l}
q \\
n
\end{array}\right)\right)(\mp \mathrm{i} \pi)^{n-q} \log ^{q} z, \quad \text { for } \theta= \pm \omega / 2, \\
h_{n-1} & =-\alpha_{n} \frac{e^{ \pm \mathrm{i} \omega}\left(\ell^{\prime}+1\right)}{\ell^{\prime}+1} \bar{z}^{m} \sum_{q=0}^{n-1}\left(\begin{array}{l}
q \\
n
\end{array}\right) \log ^{q} \bar{z}( \pm \mathrm{i} \omega)^{n-q} \\
& -b^{\prime} \bar{z}^{m} \sum_{q=0}^{n-1}\left(( \pm \mathrm{i} \pi) \lambda\left(\begin{array}{c}
q \\
n
\end{array}\right)+n\left(\begin{array}{c}
q \\
n-1
\end{array}\right)\right)( \pm \mathrm{i} \pi)^{n-1-q} \log ^{q} \bar{z} \\
& -a^{\prime} \bar{z}^{m} \sum_{q=0}^{n-1}\left(( \pm \mathrm{i} \pi) \lambda\left(\begin{array}{c}
q \\
n+1
\end{array}\right)+(n+1)\left(\begin{array}{c}
q \\
n
\end{array}\right)\right)( \pm \mathrm{i} \pi)^{n-q} \log ^{q} \bar{z}, \quad \text { for } \theta= \pm \omega / 2 .
\end{aligned}
$$

For $n=0$, the calculation is exact, meaning that $f_{-1}, g_{-1}$, and $h_{-1}$ equal zero.

Before proving the above proposition, we first show that in the case:

$$
\ell=\lambda-1, \quad \text { and } \quad \alpha_{n} \neq 0,
$$

we can reduce to Proposition A.4. A direct calculation based on formula (68) in the case when $\ell=\lambda-1$ proves that there holds

Lemma A.5. If $\ell=\lambda-1$ and $\alpha_{n} \neq 0$, the shadow $\left(V_{n}, W_{n}\right)$ solution to problem (70) writes

$$
V_{n}=\alpha_{n} v_{n}^{*}+U_{n},
$$

where $\left(U_{n}, W_{n}\right)$ satisfies

$$
\left\{\begin{aligned}
\partial_{z} \partial_{\bar{z}} U_{n} & =0, & & \text { in } \mathcal{S}_{-}, \\
\partial_{z_{+}} \partial_{\bar{z}_{+}} W_{n} & =0, & & \text { in } \mathcal{S}_{+}, \\
\partial_{z} U_{n}+\partial_{z_{+}} W_{n} & =g_{n}-\alpha_{n} z^{-1} \frac{\bar{z}^{\ell+1}}{\ell+1} \log ^{n} z, & & \text { on } \mathcal{G} \\
\partial_{\bar{z}} U_{n}+\partial_{\bar{z}_{+}} W_{n} & =h_{n}-\frac{\alpha_{n}}{n+1} \bar{z}^{\ell} \log ^{n+1} z, & & \text { on } \mathcal{G},
\end{aligned}\right.
$$

As a consequence of Proposition A.4 and Lemma A.5 we find:

Corollary A.6. Let $\lambda \neq 0, \ell \in \mathbb{N}$ and $n \in \mathbb{N}$ be chosen. Let $\left(f_{n}, g_{n}, h_{n}\right)$ be defined by (69). Then the shadow $\left(V_{n}, W_{n}\right)$ generated by $\left(f_{n}, g_{n}, h_{n}\right)$ belongs to $\mathbb{S}_{\ell+1, n+1}^{\lambda}$. An analytic formula for $\left(V_{n}, W_{n}\right)$ can be recursively deduced from Proposition A.4 and Lemma A.5.

Proof. If $\ell \neq \lambda-1$, we observe that problem (74) involves source terms whose power in $\log z$ is $n-1$, therefore pushing forward the reasoning we can decrease the power of the logarithmic terms of the source up to zero, and then solve exactly the last problem with no logarithm.

If $\ell=\lambda-1$, we first use Lemma A.5 and reduce to the situation of Proposition A.4 with the difference that a real non-zero coefficient $\gamma_{n+1}$ then appears. Examining the formulas given in Proposition A.4, we can see that even with this non-zero $\gamma_{n+1}$, the solution $\left(\alpha_{n} v_{n}^{*}+v_{n}, w_{n}\right)$ belongs to $\mathbb{S}_{\ell+1, n+1}^{\lambda}$. 
Proof of Proposition A.4. Observe that $v_{n}^{*}$ satisfies

$$
\begin{aligned}
& \partial_{z} \partial_{\bar{z}} v_{n}^{*}=f_{n}^{*}+\frac{n}{\ell^{\prime}+1} f_{n-1}^{*}, \\
& \partial_{z} v_{n}^{*}=z^{\ell^{\prime}} \frac{\bar{z}^{\ell+1}}{\ell+1} \log ^{n} z+n \frac{z^{\ell^{\prime}}}{\ell^{\prime}+1} \frac{\bar{z}^{\ell+1}}{\ell+1} \log ^{n-1} z \quad \text { and } \quad \partial_{\bar{z}} v_{n}^{*}=\frac{z^{\ell^{\prime}+1}}{\ell^{\prime}+1} \bar{z}^{\ell} \log ^{n} z
\end{aligned}
$$

To prove the proposition, we derive a particular solution $(v, w)$ for the congruence relation $\equiv$ to the following problem

$$
\left\{\begin{array}{l}
\partial_{z} \partial_{\bar{z}} v \equiv 0, \quad \text { in } \mathcal{S}_{-}, \\
\partial_{z_{+}} \partial_{\bar{z}_{+}} w \equiv 0, \quad \text { in } \mathcal{S}_{+}, \\
\partial_{z} v+\partial_{z_{+}} w \equiv-\alpha_{n} z^{\ell^{\prime}} \frac{\bar{z}^{\ell+1}}{\ell+1} \log ^{n} z+g_{n}, \quad \text { on } \mathcal{G} \\
\partial_{\bar{z}} v+\partial_{\bar{z}_{+}} w \equiv-\alpha_{n} \frac{z^{\ell^{\prime}+1}}{\ell^{\prime}+1} \bar{z}^{\ell} \log ^{n} z+h_{n}, \quad \text { on } \mathcal{G} .
\end{array}\right.
$$

Note that if $\left(v_{n}, w_{n}\right)$ given by (72) is a particular solution to (77) for the congruence relation $\equiv$, then careful calculations show that the couple $\left(V_{n-1}, W_{n-1}\right)$ given by (73) satisfies (74), which will end the proof of the proposition.

Derive now a particular solution to (77) for the congruence relation $\equiv$. For $z=r e^{ \pm i \omega / 2}$, there holds

$$
\begin{aligned}
z^{\ell^{\prime}} \frac{\bar{z}^{\ell+1}}{\ell+1} \log ^{n} z & =\frac{e^{\mp i \omega(\ell+1)}}{\ell+1} z^{m} \log ^{n} z, \\
z^{\ell^{\prime}+1} \frac{\bar{z}^{\ell}}{\ell^{\prime}+1} \log ^{n} z & =\frac{e^{ \pm i \omega\left(\ell^{\prime}+1\right)}}{\ell^{\prime}+1} \bar{z}^{m} \log ^{n} \bar{z}+\frac{e^{ \pm i \omega\left(\ell^{\prime}+1\right)}}{\ell^{\prime}+1} \bar{z}^{m} \sum_{q=0}^{n-1}\left(\begin{array}{c}
q \\
n
\end{array}\right) \log ^{q} \bar{z}( \pm i \omega)^{n-q} .
\end{aligned}
$$

Hence, denoting by $\left(c, c^{\prime}\right)$ the complex numbers given by

$$
c=\beta_{n}-\alpha_{n} \frac{e^{-i \omega(\ell+1)}}{\ell+1}, \quad c^{\prime}=\gamma_{n}-\alpha_{n} \frac{e^{i \omega\left(\ell^{\prime}+1\right)}}{\ell^{\prime}+1},
$$

the transmission conditions of (77) across $\mathcal{G}$ reads

$$
\begin{array}{lr}
\partial_{z} v+\partial_{z_{+}} w \equiv c z^{m} \log ^{n} z & \text { for } \theta=\omega / 2, \\
\partial_{z} v+\partial_{z_{+}} w \equiv \bar{c} z^{m} \log ^{n} z & \text { for } \theta=-\omega / 2, \\
\partial_{\bar{z}} v+\partial_{\bar{z}_{+}} w \equiv c^{\prime} \bar{z}^{m} \log ^{n} \bar{z} & \text { for } \theta=\omega / 2, \\
\partial_{\bar{z}} v+\partial_{\bar{z}_{+}} w \equiv \bar{c}^{\prime} \bar{z}^{m} \log ^{n} \bar{z} & \text { for } \theta=-\omega / 2 .
\end{array}
$$

Referring to Section 4.2 , we assume that $(v, w)$ writes

$$
\begin{cases}v(z) & =a z^{\lambda} \log ^{n+1} z+a^{\prime} \bar{z}^{\lambda} \log ^{n+1} \bar{z}+b z^{\lambda} \log ^{n} z, \\ (-1)^{\lambda} w\left(z_{+}\right) & =a z_{+}^{\lambda} \log ^{n+1} z_{+}+a^{\prime} \bar{z}_{+}^{\lambda} \log ^{n+1} \bar{z}_{+}+b^{\prime} \bar{z}_{+}^{\lambda} \log ^{n} \bar{z}_{+},\end{cases}
$$

with $\lambda=m+1$. Then, the following properties hold on $\mathcal{G}$ :

$$
\begin{cases}\partial_{z} v-a \lambda z^{m} \log ^{n+1} z-a(n+1) z^{m} \log ^{n} z-b \lambda z^{m} \log ^{n} z=b n z^{m} \log ^{n-1} z & \in \mathbb{J}_{n-1}^{m}, \\ (-1)^{\lambda} \partial_{z_{+}} w-a \lambda z_{+}^{m} \log ^{n+1} z_{+}-a(n+1) z_{+}^{m} \log ^{n} z_{+}=0 & \in \mathbb{J}_{n-1}^{m},\end{cases}
$$


and similarly

$$
\begin{cases}\partial_{\bar{z}} v-a^{\prime} \lambda \bar{z}^{m} \log ^{n+1} \bar{z}-a^{\prime}(n+1) \bar{z}^{m} \log ^{n} \bar{z}=0 & \in \mathbb{J}_{n-1}^{m}, \\ (-1)^{\lambda} \partial_{\bar{z}_{+}} w-a^{\prime} \lambda \bar{z}_{+}^{m} \log ^{n+1} \bar{z}_{+}-a^{\prime}(n+1) \bar{z}_{+}^{m} \log ^{n} \bar{z}_{+}-b^{\prime} \lambda \bar{z}_{+}^{m} \log ^{n} \bar{z}_{+}=b^{\prime} n \bar{z}_{+}^{m} \log ^{n-1} \bar{z}_{+} \in \mathbb{J}_{n-1}^{m} .\end{cases}
$$

Recall that $z_{+}=-z$. Due to the branch cut on $\mathbb{R}^{-}$of log, we have to distinguish the two branches of $\mathcal{G}$ given by $\theta=\omega / 2$ and $\theta=-\omega / 2$. Actually, for $\theta= \pm \omega / 2$, since $\theta_{+}= \pm \omega / 2- \pm \pi$, we infer for $\tilde{n} \in\{n+1, n\}$

$$
z^{m} \log ^{\tilde{n}} z+(-1)^{\lambda} z_{+}^{m} \log ^{\tilde{n}} z_{+}= \pm \mathrm{i} \pi \tilde{n} z^{m} \log ^{\tilde{n}-1} z-z^{m} \sum_{q=0}^{\tilde{n}-2}\left(\begin{array}{c}
q \\
\tilde{n}
\end{array}\right)(\mp \mathrm{i} \pi)^{\tilde{n}-q} \log ^{q} z, \quad \text { for } \theta= \pm \omega / 2
$$

Therefore

$$
\partial_{z} v+\partial_{z_{+}} w= \pm \mathrm{i} \pi a \lambda(n+1) z^{m} \log ^{n} z+b \lambda z^{m} \log ^{n} z+g_{n-1}, \quad \text { for } \theta= \pm \omega / 2
$$

where $g_{n-1}$ equals

$g_{n-1}=b n z^{m} \log ^{n-1} z-a z^{m} \sum_{q=0}^{n-1}\left((\mp \mathrm{i} \pi) \lambda\left(\begin{array}{c}q \\ n+1\end{array}\right)+(n+1)\left(\begin{array}{l}q \\ n\end{array}\right)\right)(\mp \mathrm{i} \pi)^{n-q} \log ^{q} z, \quad$ for $\theta= \pm \omega / 2$.

We thus infer $g_{n-1} \in \mathbb{J}_{n-1}^{m}$. Therefore, the two first congruence relations in (78) are satisfied iff

$$
\begin{aligned}
\mathrm{i} \pi a \lambda(n+1)+b \lambda & =c, \\
-\mathrm{i} \pi a \lambda(n+1)+b \lambda & =\bar{c} .
\end{aligned}
$$

This system of two equations has a unique solution $(a, b) \in \mathbb{R}^{2}$ given by

$$
a=\frac{1}{\pi \lambda(n+1)} \operatorname{Im}(c), \quad b=\frac{1}{\lambda} \operatorname{Re}(c),
$$

hence

$$
a=\frac{1}{\pi \lambda(n+1)}\left(\operatorname{Im} \beta_{n}+\alpha_{n} \frac{\sin \omega(\ell+1)}{(\ell+1)}\right), \quad b=\frac{1}{\lambda}\left(\operatorname{Re} \beta_{n}-\alpha_{n} \frac{\cos \omega(\ell+1)}{(\ell+1)}\right) .
$$

Similar computations imply that

$$
\partial_{\bar{z}} v+\partial_{\bar{z}_{+}} w=\mp \mathrm{i} \pi a^{\prime} \lambda(n+1) \bar{z}^{m} \log ^{n} \bar{z}-b^{\prime} \lambda \bar{z}^{m} \log ^{n} \bar{z}+h_{n-1}, \quad \text { for } \theta= \pm \omega / 2,
$$

where $h_{n-1}$ equals

$$
\begin{aligned}
h_{n-1} & =-\alpha_{n} \frac{e^{ \pm \mathrm{i} \omega\left(\ell^{\prime}+1\right)}}{\ell^{\prime}+1} \bar{z}^{m} \sum_{q=0}^{n-1}\left(\begin{array}{l}
q \\
n
\end{array}\right) \log ^{q} \bar{z}( \pm \mathrm{i} \omega)^{n-q} \\
& -b^{\prime} \bar{z}^{m} \sum_{q=0}^{n-1}\left(( \pm \mathrm{i} \pi) \lambda\left(\begin{array}{l}
q \\
n
\end{array}\right)+n\left(\begin{array}{c}
q \\
n-1
\end{array}\right)\right)( \pm \mathrm{i} \pi)^{n-1-q} \log ^{q} \bar{z} \\
& -a^{\prime} \bar{z}^{m} \sum_{q=0}^{n-1}\left(( \pm \mathrm{i} \pi) \lambda\left(\begin{array}{c}
q \\
n+1
\end{array}\right)+(n+1)\left(\begin{array}{l}
q \\
n
\end{array}\right)\right)( \pm \mathrm{i} \pi)^{n-q} \log ^{q} \bar{z}, \quad \text { for } \theta= \pm \omega / 2 .
\end{aligned}
$$


Therefore, the third and fourth equations in (78) write on $\mathcal{G}$ as

$$
\begin{aligned}
-\mathrm{i} \pi a^{\prime} \lambda(n+1) \bar{z}^{m} \log ^{n} \bar{z}-b^{\prime} \lambda \bar{z}^{m} \log ^{n} \bar{z} \equiv c^{\prime} \bar{z}^{m} \log ^{n} \bar{z}, & \text { for } \theta=\omega / 2, \\
\mathrm{i} \pi a^{\prime} \lambda(n+1) \bar{z}^{m} \log ^{n} \bar{z}-b^{\prime} \lambda \bar{z}^{m} \log ^{n} \bar{z} \equiv \bar{c}^{\prime} \bar{z}^{m} \log ^{n} \bar{z}, & \text { for } \theta=-\omega / 2 .
\end{aligned}
$$

Thus, the couple $\left(a^{\prime}, b^{\prime}\right)$ is unique, in $\mathbb{R}^{2}$ and satisfies

$$
\begin{aligned}
\mathrm{i} \pi a^{\prime} \lambda(n+1)+b^{\prime} \lambda & =-c^{\prime}, \\
-\mathrm{i} \pi a^{\prime} \lambda(n+1)+b^{\prime} \lambda & =-\bar{c}^{\prime},
\end{aligned}
$$

hence

$$
a^{\prime}=\frac{1}{\pi \lambda(n+1)}\left(-\operatorname{lm} \gamma_{n}+\alpha_{n} \frac{\sin \omega\left(\ell^{\prime}+1\right)}{\left(\ell^{\prime}+1\right)}\right), \quad b^{\prime}=\frac{1}{\lambda}\left(-\operatorname{Re} \gamma_{n}+\alpha_{n} \frac{\cos \omega\left(\ell^{\prime}+1\right)}{\left(\ell^{\prime}+1\right)}\right),
$$

and therefore, $\left(v_{n}, w_{n}\right)$ given by (72) is a particular solution to (77), and satisfies the assertions of the proposition by constructions, which ends the proof.

\section{A.2.2 The case $\lambda=0$}

We consider now the case $\lambda=0$, which corresponds to a source term in $\mathrm{T}^{-2}$, according to Lemma 3.1. As seen previously at Proposition 4.5, it is necessary to increase the power of the logarithm terms of the solution by 1 , in comparison with the case $\lambda \neq 0$. Moreover, observe that a constant term may appear, as in Proposition 4.5 for instance, and it is necessary to consider problem (54) to ensure the continuity of the solution across $\mathcal{G}$. The boundary data are now $\widetilde{g}_{n}$ and $\widetilde{h}_{n+1}$ defined as

$\forall z \in \mathcal{G}, \quad \widetilde{g}_{n}(z)=\left\{\begin{array}{ll}\beta_{n} \log ^{n} z, & \text { for } \theta=\omega / 2, \\ \overline{\beta_{n}} \log ^{n} z, & \text { for } \theta=-\omega / 2,\end{array} \quad\right.$ and $\quad \tilde{h}_{n+1}(z)= \begin{cases}\gamma_{n+1} \log ^{n+1} z, & \text { for } \theta=\omega / 2, \\ \overline{\gamma_{n+1}} \log ^{n+1} z, & \text { for } \theta=-\omega / 2,\end{cases}$ where $n \in \mathbb{N}, \beta_{n} \in \mathbb{C}$, and $\gamma_{n+1} \in \mathbb{C}$. The problem which we want to solve is, instead of (70),

$$
\begin{cases}\partial_{z} \partial_{\bar{z}} V_{n}=f_{n}, & \text { in } \mathcal{S}_{-}, \\ \partial_{z_{+}} \partial_{\bar{z}_{+}} W_{n}=0, & \text { in } \mathcal{S}_{+}, \\ z \partial_{z} V_{n}-\bar{z} \partial_{\bar{z}} V_{n}-z_{+} \partial_{z_{+}} W_{n}+\bar{z}_{+} \partial_{\bar{z}_{+}} W_{n}=\widetilde{g}_{n} & \text { on } \mathcal{G}, \\ V_{n}-W_{n}=\widetilde{h}_{n+1} & \text { on } \mathcal{G} .\end{cases}
$$

We have the following proposition.

Proposition A.7. Let $(\ell, n) \in \mathbb{N} \times(\mathbb{N} \backslash\{0\})$. Note that, according to (71), $\ell^{\prime}=-2-\ell$. Define $\left(v_{n}, w_{n}\right)$ as

$$
\left\{\begin{array}{l}
v_{n}(z)=a \log ^{n+2} z+a^{\prime} \log ^{n+2} \bar{z}+b \log ^{n+1} z \\
w_{n}\left(z_{+}\right)=a \log ^{n+2} z_{+}+a^{\prime} \log ^{n+2} \bar{z}_{+}+b^{\prime} \log ^{n+1} \bar{z}_{+}
\end{array}\right.
$$

where

$$
\begin{aligned}
& \left\{\begin{array}{l}
a=\frac{1}{2 \pi(n+2)}\left(\frac{1}{n+1}\left(\operatorname{Im} \beta_{n}+2 \alpha_{n} \frac{\sin \omega(\ell+1)}{(\ell+1)}\right)+\operatorname{Im} \gamma_{n+1}\right) \\
b=\frac{1}{2}\left(\frac{1}{n+1}\left(\operatorname{Re} \beta_{n}-2 \alpha_{n} \frac{\cos \omega(\ell+1)}{(\ell+1)}\right)+\operatorname{Re} \gamma_{n+1}\right)
\end{array}\right. \\
& \left\{\begin{array}{l}
a^{\prime}=\frac{1}{2 \pi(n+2)}\left(\frac{1}{n+1}\left(\operatorname{Im} \beta_{n}+2 \alpha_{n} \frac{\sin \omega(\ell+1)}{(\ell+1)}\right)-\operatorname{Im} \gamma_{n+1}\right) \\
b^{\prime}=\frac{1}{2}\left(\frac{1}{n+1}\left(\operatorname{Re} \beta_{n}-2 \alpha_{n} \frac{\cos \omega(\ell+1)}{(\ell+1)}\right)-\operatorname{Re} \gamma_{n+1}\right)
\end{array}\right.
\end{aligned}
$$


We recall that according to (68), for $\lambda=0$ :

$$
v_{n}^{*}=-\frac{1}{(\ell+1)^{2}}(\bar{z} / z)^{\ell+1} \log ^{n} z
$$

Then, $\left(\alpha_{n} v_{n}^{*}+v_{n}, w_{n}\right)$ is a particular solution to (80) for the congruence relation $\equiv$. This means that the couple of functions $\left(V_{n-1}, W_{n-1}\right)$ defined by

$$
V_{n-1}=V_{n}-\left(\alpha_{n} v_{n}^{*}+v_{n}\right), \quad W_{n-1}=W_{n}-w_{n}
$$

satisfies the following problem:

$$
\begin{cases}\partial_{z} \partial_{\bar{z}} V_{n-1}=f_{n-1}, & \text { in } \mathcal{S}_{-} \\ \partial_{z_{+}} \partial_{\bar{z}_{+}} W_{n-1}=0, & \text { in } \mathcal{S}_{+} \\ z \partial_{z} V_{n-1}-\bar{z} \partial_{\bar{z}} V_{n-1}-z_{+} \partial_{z_{+}} W_{n-1}+\bar{z}_{+} \partial_{\bar{z}_{+}} W_{n-1}=\widetilde{g}_{n-1} & \text { on } \mathcal{G} \\ V_{n-1}-W_{n-1}=\widetilde{h}_{n} & \text { on } \mathcal{G}\end{cases}
$$

where

$$
\left(f_{n-1}, \widetilde{g}_{n-1}, \widetilde{h}_{n}\right) \in \mathbb{S}_{\ell, n-1}^{-2,-} \times \mathbb{J}_{n-1}^{0} \times \mathbb{J}_{n}^{0} .
$$

More precisely we have the explicit formulas

$$
\begin{aligned}
f_{n-1} & =-\frac{n \alpha_{n}}{\ell^{\prime}+1} f_{n-1}^{*}, \\
\widetilde{g}_{n-1} & =(n+2) a \sum_{q=0}^{n-1}\left(\begin{array}{c}
q \\
n+1
\end{array}\right)(\mp \mathrm{i} \pi)^{n+1-q} \log ^{q} z-(n+2) a^{\prime} \sum_{q=0}^{n-1}\left(\begin{array}{c}
q \\
n+1
\end{array}\right)( \pm \mathrm{i} \pi)^{n+1-q} \log ^{q} \bar{z} \\
& -(n+1) b^{\prime} \sum_{q=0}^{n-1}\left(\begin{array}{c}
q \\
n+1
\end{array}\right)( \pm \mathrm{i}(\pi-\omega))^{n+1-q} \log ^{q} z-\frac{n \alpha_{n} e^{\mp i \omega(\ell+1)}}{(\ell+1)^{2}} \log ^{n-1} z, \\
\widetilde{h}_{n} & =a \sum_{q=0}^{n}\left(\begin{array}{c}
q \\
n+2
\end{array}\right)(\mp \mathrm{i} \pi)^{n+2-q} \log ^{q} z+a^{\prime} \sum_{q=0}^{n}\left(\begin{array}{c}
q \\
n+2
\end{array}\right)( \pm \mathrm{i} \pi)^{n+2-q} \log ^{q} \bar{z} \\
& +b^{\prime} \sum_{q=0}^{n-1}\left(\begin{array}{c}
q \\
n+1
\end{array}\right)( \pm \mathrm{i}(\pi-\omega))^{n+1-q} \log ^{q} z+\frac{\alpha_{n} e^{\mp i \omega(\ell+1)}}{(\ell+1)^{2}} \log ^{n} z .
\end{aligned}
$$

Proof. Writing $z \partial_{z} v_{\ell, n}-\bar{z} \partial_{\bar{z}} v_{\ell, n}$ and $z_{+} \partial_{z_{+}} w_{\ell, n}-\bar{z}_{+} \partial_{\bar{z}_{+}} w_{\ell, n}$ and identifying the term in $\log ^{n} z$, we infer

$$
\begin{aligned}
& \pi(n+2)(n+1)\left(a+a^{\prime}\right)=\operatorname{Im} \beta_{n}+2 \alpha_{n} \frac{\sin \omega(\ell+1)}{\ell+1}, \\
& (n+1)\left(b+b^{\prime}\right)=\operatorname{Re} \beta_{n}-2 \alpha_{n} \frac{\cos \omega(\ell+1)}{\ell+1} .
\end{aligned}
$$

Then, writing the condition $v_{\ell, n}+\alpha_{n} v_{n}^{*}-w_{\ell, n}=\widetilde{h}_{n+1}$ and identifying the term in $\log ^{n+1} z$, we obtain

$$
\begin{aligned}
& a-a^{\prime}=\frac{1}{\pi(n+2)} \operatorname{lm} \gamma_{n+1}, \\
& b-b^{\prime}=\operatorname{Re} \gamma_{n+1},
\end{aligned}
$$

hence the proposition with $\widetilde{g}_{n-1}$ and $\widetilde{h}_{n}$ given by (84b) and (84c). 
For $n=0$, we solve exactly the following problem

$$
\begin{aligned}
& \partial_{z} \partial_{\bar{z}} V_{\ell, 0}=\frac{-\alpha_{\ell, 0}}{(\ell+1)^{2}}(\bar{z} / z)^{\ell+1}, \\
& \partial_{z} \partial_{\bar{z}} W_{\ell, 0}=0, \\
& V_{\ell, 0}-W_{\ell, 0}=\left\{\begin{array}{l}
\gamma_{1} \log z+\gamma_{0}, \text { if } \theta=\omega / 2, \\
\bar{\gamma}_{1} \log z+\bar{\gamma}_{0}, \text { if } \theta=-\omega / 2,
\end{array}\right. \\
& \left(z \partial_{z} V_{\ell, 0}-\bar{z} \partial_{\bar{z}} V_{\ell, 0}\right)-\left(z_{+} \partial_{z_{+}} W_{\ell, 0}-\bar{z}_{+} \partial_{\bar{z}_{+}} W_{\ell, 0}\right)=\left\{\begin{array}{l}
\beta_{0}, \text { if } \theta=\omega / 2, \\
\bar{\beta}_{0}, \text { if } \theta=\omega / 2,
\end{array}\right.
\end{aligned}
$$

thanks to the following proposition.

Proposition A.8. Let $\ell \in \mathbb{N}$. Recall that, in this case,

$$
v_{\ell, 0}^{*}=-\frac{1}{(\ell+1)^{2}}(\bar{z} / z)^{\ell+1} .
$$

Define $\left(v_{\ell, 0}, w_{\ell, 0}\right)$ as

$$
\left\{\begin{array}{l}
v_{\ell, 0}(z)=a \log ^{2} z+a^{\prime} \log ^{2} \bar{z}+b \log z, \\
w_{\ell, 0}\left(z_{+}\right)=a \log ^{2} z_{+}+a^{\prime} \log ^{2} \bar{z}_{+}+\tilde{b} \log z_{+}+b^{\prime} \log \bar{z}_{+}+c
\end{array}\right.
$$

where, setting

$$
d=\beta_{0}-2 \alpha_{\ell, 0} e^{-i \omega(\ell+1)} /(\ell+1),
$$

we have

$$
\left\{\begin{array}{l}
a=a^{\prime}+\frac{1}{2 \pi} \operatorname{Im} \gamma_{1}=\frac{1}{4 \pi} \operatorname{Im}\left(d+\gamma_{1}\right), \\
b^{\prime}=\frac{1}{2} \operatorname{Re}\left(d-\gamma_{1}\right) \\
\tilde{b}=\frac{\pi-\omega}{\pi} b^{\prime}-\frac{\alpha_{\ell, 0}}{\pi(\ell+1)^{2}} \sin \omega(\ell+1)+\operatorname{Im} \gamma_{0} \\
b=b^{\prime}+\tilde{b}+\operatorname{Re} \gamma_{1}, \\
c=2 \pi a(\pi-\omega)-\frac{\alpha_{\ell, 0}}{(\ell+1)^{2}} \cos \omega(\ell+1)-\frac{\pi-\omega}{2} \operatorname{Im} \gamma_{1}-\operatorname{Re} \gamma_{0} .
\end{array}\right.
$$

Then, $\left(\alpha_{\ell, 0} v_{\ell, 0}^{*}+v_{\ell, 0}, w_{\ell, 0}\right)$ is a particular exact solution to (85).

Proof. The transmission conditions of (85) across $\mathcal{G}$ writes

$$
\left(z \partial_{z} v_{\ell, 0}-\bar{z} \partial_{\bar{z}} v_{\ell, 0}\right)-\left(z_{+} \partial_{z_{+}} w_{\ell, 0}-\bar{z}_{+} \partial_{\bar{z}_{+}} w_{\ell, 0}\right)= \pm 2 i \pi\left(a+a^{\prime}\right)+b-\tilde{b}+b^{\prime}
$$

Since

we infer that

$$
d=\beta_{0}-2 \alpha_{\ell, 0} \frac{e^{-i \omega(\ell+1)}}{\ell+1}
$$

$$
\begin{aligned}
2 \mathrm{i} \pi\left(a+a^{\prime}\right)+b-\tilde{b}+b^{\prime} & =d, \\
-2 \mathrm{i} \pi\left(a+a^{\prime}\right)+b-\tilde{b}+b^{\prime} & =\bar{d} .
\end{aligned}
$$

RR n ${ }^{\circ} 8204$ 
Since

$v_{\ell, 0}-w_{\ell, 0}=a\left( \pm 2 i \pi \log z+\pi^{2}\right)+a^{\prime}\left(\mp 2 i \pi \log z-2 \pi \omega+\pi^{2}\right)+\left(b-\tilde{b}-b^{\prime}\right) \log z \pm \mathrm{i}\left(\pi \tilde{b}-(\pi-\omega) b^{\prime}\right)-c$,

the continuity across $\mathcal{G}$ implies

$$
\begin{aligned}
& \pm 2 \mathrm{i} \pi\left(a-a^{\prime}\right)+\left(b-\tilde{b}-b^{\prime}\right)=\left\{\begin{array}{l}
\gamma_{1}, \text { if } \theta=\omega / 2, \\
\bar{\gamma}_{1}, \text { if } \theta=-\omega / 2,
\end{array}\right. \\
& \pi^{2} a+\pi(\pi-2 \omega) a^{\prime} \pm \mathrm{i}\left(\pi \tilde{b}-(\pi-\omega) b^{\prime}\right)-c=\frac{\alpha_{\ell, 0}}{(\ell+1)^{2}} e^{\mp i \omega(\ell+1)}+\left\{\begin{array}{l}
\gamma_{0}, \text { if } \theta=\omega / 2, \\
\bar{\gamma}_{0}, \text { if } \theta=-\omega / 2,
\end{array}\right.
\end{aligned}
$$

hence

$$
a=a^{\prime}+\frac{1}{2 \pi} \operatorname{Im} \gamma_{1}, \quad b-\tilde{b}-b^{\prime}=\operatorname{Re} \gamma_{1}
$$

which ends the proof.

\section{A.3 Formal derivation of the singularities}

\section{A.3.1 Principles}

We are now ready to present how to obtain the singularities.

- First, choose the leading (primal or dual) singularity: $z^{k}, k \in \mathbb{Z}$ or $\log z$.

- For $z^{k}$, use Proposition 4.3, Proposition 4.4 or Proposition 4.5 depending whether $k \in\{-1,-2\}$ or not, and for $\log z$ use Proposition 4.6, in order to obtain the first shadow term. This shadow is composed of terms as $z^{k+2-\ell} \bar{z}^{\ell} \log ^{n} z$, with $n=1$ or 2 and $\ell \in\{0,1,2\}$.

- If $k+2 \neq-2$, check whether $k+2-\ell=0$, and apply recursively Proposition A.4 or Corollary A.5 for each term of the shadow. If $k+2=-2$, apply recursively Proposition A.7 and then Proposition A.8.

- Repeat the process till the desired order of the shadow.

- Sum all the terms.

\section{A.3.2 Generic expression of the shadows at any order}

The results of subsection A.2 leads to the following proposition, that provides the generic expression of the primal singularities of $\mathscr{L}_{\zeta}$.

Proposition A.9. Let $k \in \mathbb{N}$. We recall that according to (48), $s_{0}^{k}=z^{k}$. For $j \geqslant 1$, the function $\mathrm{s}_{j}^{k}=\left(\chi_{j}^{k}, \xi_{j}^{k}\right)$ defined by (52), which is the shadow of order $j$ of $\mathrm{s}_{0}^{k}$, writes

$$
\begin{aligned}
\chi_{j}^{k}(z)=a_{k j} z^{k+2 j} \log ^{j} z+ & a_{k j}^{\prime} \bar{z}^{k+2 j} \log ^{j} \bar{z} \\
& +\sum_{n=0}^{j-1} \sum_{m=0}^{j-n} b_{k j, n m} z^{k+2 j-m} \bar{z}^{m} \log ^{n} z+\sum_{n=0}^{j-1} \sum_{m=0}^{j-n} c_{k j, n m} z^{m} \bar{z}^{k+2 j-m} \log ^{n} \bar{z},
\end{aligned}
$$




$$
\begin{aligned}
\xi_{j}^{k}\left(z_{+}\right)=(-1)^{k} a_{k j} z_{+}^{k+2 j} & \log ^{j} z_{+}+(-1)^{k} a_{k j}^{\prime} \bar{z}_{+}^{k+2 j} \log ^{j} \bar{z}_{+} \\
& +\sum_{n=0}^{j-1} \sum_{m=0}^{j-n} b_{k j, n m}^{\prime} z_{+}^{k+2 j-m} \bar{z}_{+}^{m} \log ^{n} z_{+}+\sum_{n=0}^{j-1} \sum_{m=0}^{j-n} c_{k j, n m}^{\prime} z_{+}^{m} \bar{z}_{+}^{k+2 j-m} \log ^{n} \bar{z}_{+},
\end{aligned}
$$

where the coefficients $a_{k j}, a_{k j}^{\prime}, b_{k j, n m}, b_{k j, n m}^{\prime}, c_{k j, n m}$, and $c_{k j, n m}^{\prime}$ are real coefficients. These coefficients are obtained by the induction process given by Proposition A.4.

From this proposition, explicit the primal singular functions $\mathfrak{S}^{k, p}$ of corner asymptotics (4) can be easily obtained. Actually, according to (18), it is sufficient to make explicit the functions $\mathfrak{s}_{j}^{k, p}$, which are given by

$$
\mathfrak{s}_{j}^{k, p}(r, \theta)= \begin{cases}\operatorname{Re}\left(\chi_{j}^{k}\left(r e^{i \theta}\right)\right) \delta_{0}^{p}+\operatorname{Im}\left(\chi_{j}^{k}\left(r e^{i \theta}\right)\right) \delta_{1}^{p}, & \text { if }|\theta| \leqslant \omega / 2, \\ \operatorname{Re}\left(\xi_{j}^{k}\left(r e^{i \theta_{+}}\right)\right) \delta_{0}^{p}+\operatorname{Im}\left(\xi_{j}^{k}\left(r e^{i \theta_{+}}\right)\right) \delta_{1}^{p}, & \text { elsewhere },\end{cases}
$$

according to (51), where $\delta_{0}^{p}$ and $\delta_{1}^{p}$ are Kronecker symbols. We remind that $\theta_{+}$is defined by

$$
\theta_{+}=\left\{\begin{array}{l}
\theta-\pi, \text { if } \theta \in(0, \pi] \\
\theta+\pi, \text { if } \theta \in[-\pi, 0)
\end{array}\right.
$$

Then, we develop relation (18) with respect to the power of the logarithmic term $\log r$, and we identify the obtained expressions with the previous expression (22) of $\mathfrak{S}^{k, p}(r, \theta)$.

For instance, let us determine $\Phi_{j, n}^{k, 0}$ in the sector $\mathcal{S}_{-}$, for $0 \leqslant n \leqslant j$. Using (88), after tedious calculus, we obtain:

$$
\Phi_{j, j}^{k, 0-}(\theta)=\left(a_{k j}+a_{k j}^{\prime}\right) \cos (k+2 j) \theta, \quad \text { for } n=j,
$$

and for any $n \leqslant j-1$,

$$
\begin{aligned}
\Phi_{j, n}^{k, 0-}(\theta) & =\left(\begin{array}{c}
n \\
j
\end{array}\right) \theta^{j-n}\left(a_{k j}+a_{k j}^{\prime}\right) \cos \left((k+2 j) \theta+\frac{\pi}{2}(j-n)\right) \\
& +\sum_{q=0}^{n}\left(\begin{array}{c}
q \\
n-q
\end{array}\right) \theta^{n-2 q} \sum_{m=0}^{j+q-n}\left(b_{k j, n-q m}+c_{k j, n-q m}\right) \cos \left((k+2 j-2 m) \theta+\frac{\pi}{2}(n-2 q)\right)
\end{aligned}
$$

A similar reasoning leads to the generic expression of the dual singularities. 


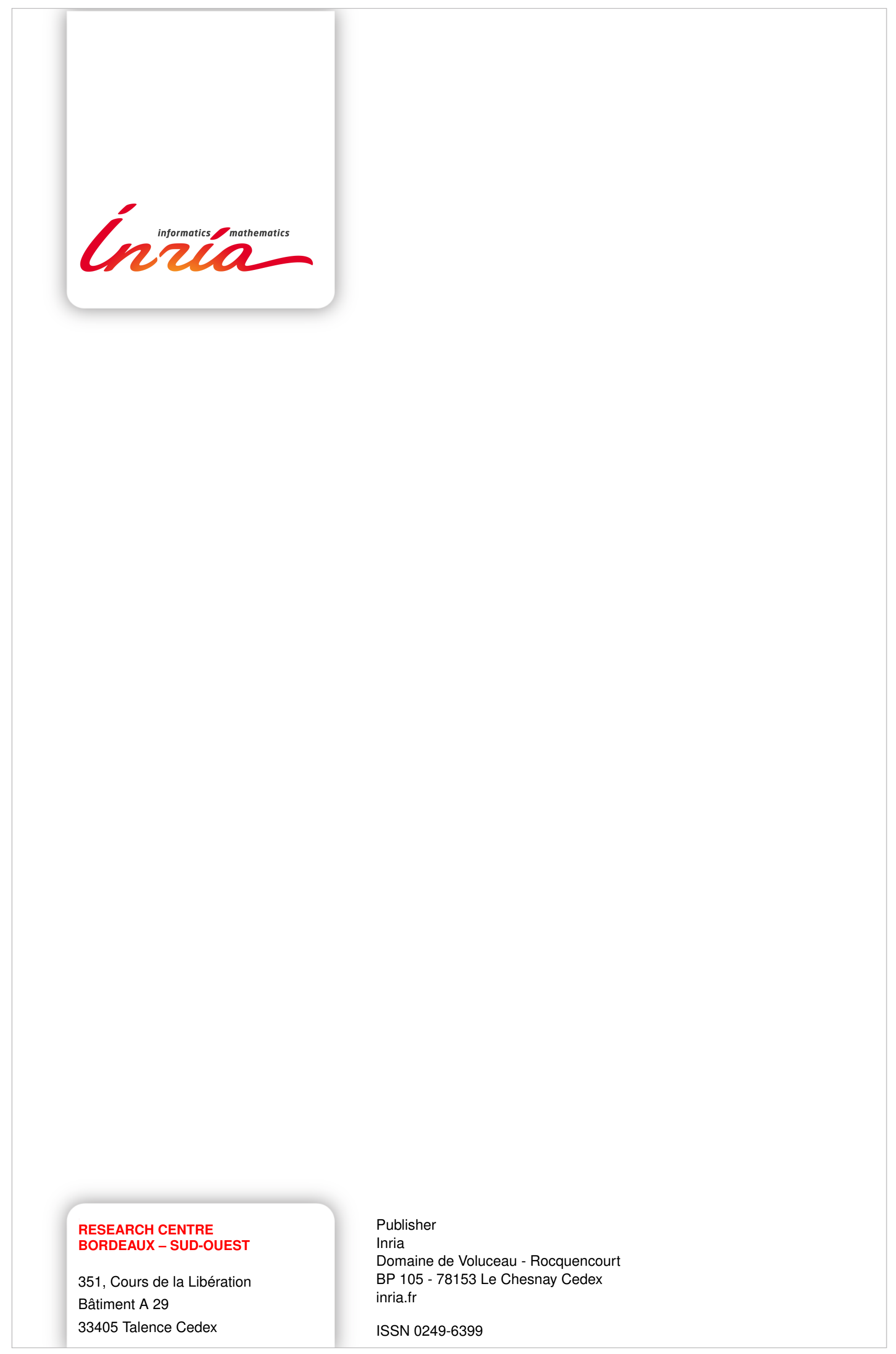

